\title{
WestVirginiaUniversity
}

THE RESEARCH REPOSITORY @ WVU

Graduate Theses, Dissertations, and Problem Reports

2010

\section{Low-Power Analog Circuits for Sub-Band Speech Processing}

Anvesh Kumar Singireddy

West Virginia University

Follow this and additional works at: https://researchrepository.wvu.edu/etd

\section{Recommended Citation}

Singireddy, Anvesh Kumar, "Low-Power Analog Circuits for Sub-Band Speech Processing" (2010). Graduate Theses, Dissertations, and Problem Reports. 4657.

https://researchrepository.wvu.edu/etd/4657

This Thesis is protected by copyright and/or related rights. It has been brought to you by the The Research Repository @ WVU with permission from the rights-holder(s). You are free to use this Thesis in any way that is permitted by the copyright and related rights legislation that applies to your use. For other uses you must obtain permission from the rights-holder(s) directly, unless additional rights are indicated by a Creative Commons license in the record and/ or on the work itself. This Thesis has been accepted for inclusion in WVU Graduate Theses, Dissertations, and Problem Reports collection by an authorized administrator of The Research Repository @ WVU. For more information, please contact researchrepository@mail.wvu.edu. 


\title{
Low-Power Analog Circuits for Sub-Band Speech Processing
}

\author{
by \\ Anvesh Kumar Singireddy \\ Thesis submitted to the \\ College of Engineering and Mineral Resources \\ at West Virginia University \\ in partial fulfillment of the requirements \\ for the degree of \\ Master of Science \\ in \\ Electrical Engineering \\ David W. Graham, Ph.D., Chair \\ Dimitris Korakakis, Ph.D. \\ Jeremy Dawson, Ph.D.
}

Lane Department of Computer Science and Electrical Engineering

Morgantown, West Virginia

2010

Keywords: Analog, VLSI, integrated circuits, speech processing, low-power, derivative

Copyright 2010 Anvesh Kumar Singireddy 


\author{
Abstract \\ Low-Power Analog Circuits for Sub-Band Speech Processing \\ by \\ Anvesh Kumar Singireddy \\ Master of Science in Electrical Engineering \\ West Virginia University \\ David W. Graham, Ph.D., Chair
}

The need for efficient electronics has been increasing by the day, as have the constraints on power and size of the devices. Also the increase in use of mobile and wearable electronics has been leading to innovative methods to conserve power and increase functionality. The traditional approach of signal processing heavily relies on the Digital Signal Processing (DSP) hardware to perform most of the tasks, which has lead to power-hungry circuits. Use of analog front-end devices could prove to be efficient, since most of the real-world data is analog and since the DSP could be spared for more application-specific tasks within the system, thereby resulting in more efficient mixed-signal systems.

The focus in this work is to develop an analog front-end for speech-processing applications with inspiration from biology, and trying to mimic human auditory perception techniques. The circuits are designed in 600nm, 350nm and 180nm CMOS processes and are biased in the sub-threshold region to consume low-power. Also, various modules of the system are connected using multiplexing circuits to allow post-fabrication reconfigurability to suit various applications. These circuits are biased using a network of floating-gate transistors which allow reconfigurability and increased bias accuracy. This thesis mainly describes two modules of the analog front-end used for speech processing: derivative circuit and voltagemode subtractor circuit, which are used for processing spectrally decomposed signals. These circuits could be used for applications like audio analysis or event detection. 


\section{Acknowledgments}

I want to thank my advisor, Dr. David Graham, for his encouragement, knowledge and support throughout my graduate studies here at West Virginia University. I also want to thank the rest of my committee, Dr. Dimitris Korakakis and Dr. Jeremy Dawson, for lending their expertise on the periphery of this research area. Thanks to GTronix for partially funding this project and for their valuable feedback. I also want to acknowledge Brandon Rumberg, who worked on the other half of this project. Also, a big thanks to my family and friends, without whom I could not have come so far. 


\section{Contents}

$\begin{array}{ll}\text { Acknowledgments } & \text { iii }\end{array}$

List of Figures $\quad$ vi

1 Introduction $\quad 1$

1.1 Analog Front-ends . . . . . . . . . . . . . . . . . . . . . . . . . . . . . . . .

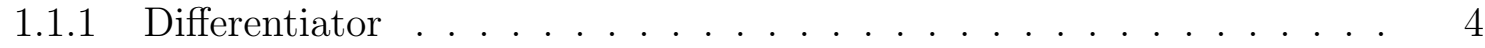

1.1 .2 Subtractor . . . . . . . . . . . . . . . 5

1.1.3 Floating-gate Transistors . . . . . . . . . . . . . . . 5

1.1.4 Filter-Bank with Sub-band Processing . . . . . . . . . . . . . 6

1.2 Basic Building Blocks . . . . . . . . . . . . . . . . . . . . . . . 6

1.3 Organization . . . . . . . . . . . . . . . . . 7

2 Derivative Circuit $\quad 9$

2.1 Different Kinds of Derivative Models . . . . . . . . . . . . . . . . . . . . 10

2.1.1 First-Order High-Pass Filter . . . . . . . . . . . . . . . . . . 10

2.1.2 Capacitor for Derivative . . . . . . . . . . . . . . . . . . . . 12

2.1.3 Current-Output Derivative Circuits . . . . . . . . . . . . . . 16

2.1.4 Differently-Clamped-Capacitor based Differentiator . . . . . . . . . 17

2.2 Design of Differentiator Circuit . . . . . . . . . . . . . . . . . . 18

2.2.1 Various Blocks of the Differentiator Circuit . . . . . . . . . . . 22

2.2.2 Results from the Differentiator Circuit . . . . . . . . . . . . . 25

3 Subtractor Circuit $\quad 29$

3.1 Basic Principle . . . . . . . . . . . . . . . . . . . . . . . . . . . . 29

3.2 Design of Subtractor Circuit . . . . . . . . . . . . . . . . . 30

3.2.1 Working of Subtractor Circuit . . . . . . . . . . . . . 31

3.2 .2 Output Limitations . . . . . . . . . . . . . . . . . . . . . . . . . . . . . . . . . . . 33

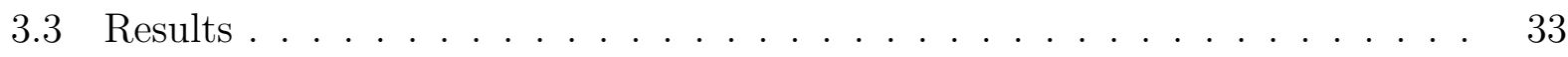

4 Floating-Gate Tranistors $\quad 41$

4.1 Floating-Gate Transistors . . . . . . . . . . . . . . . . . 41

4.1 .1 Electron Injection . . . . . . . . . . . . . . . . . . . . . 42

4.1 .2 Electron Tunneling . . . . . . . . . . . . . . . . . . . . 44

4.1 .3 Tunneling Junction . . . . . . . . . . . . . . . . . . . 46 
5 Analog Auditory Front-End $\quad 48$

5.1 Working of Analog Auditory Front-End . . . . . . . . . . . . . . 48

6 Applications and Conclusion $\quad 53$

6.1 Low-Power Hardware-Based Event Detection . . . . . . . . . . . . . . . . 53

6.2 Design of the Analog System . . . . . . . . . . . . . . . . . . . . 54

6.2.1 Single-Dimensional Sound Localization . . . . . . . . . . . . . . 54

6.2 .2 Sound/Event Classification . . . . . . . . . . . . . . . 55

6.3 Conclusion . . . . . . . . . . . . . . . . . . . 56

$\begin{array}{ll}\text { A Cadence setup } & 59\end{array}$

B More Derivative Circuit Models $\quad 60$

B.1 Hysteretic Differentiators . . . . . . . . . . . . . . . . . . . . . . 60

B.2 RL-Type Differentiators . . . . . . . . . . . . . . . . . . 61

$\begin{array}{ll}\text { References } & 64\end{array}$

$\begin{array}{ll}\text { Approval Page } & 66\end{array}$ 


\section{List of Figures}

1.1 Analog and digital systems . . . . . . . . . . . . . . . . . . . . 3

1.2 Block diagram of the analog front-end . . . . . . . . . . . . . . . 3

1.3 A real-time system with analog front-end. . . . . . . . . . . . . . . . 4

1.4 Floating-gate symbol . . . . . . . . . . . . . . . . . . . . . . . . . . . . . . . . .

1.5 Transistor symbols . . . . . . . . . . . . . . . . . 7

2.1 Differentiation of a sine wave (simulated) . . . . . . . . . . . . . . 10

2.2 Ideal frequency response of a differentiator (simulated) . . . . . . . . . . . . . 11

2.3 Variation of gain with capacitor size . . . . . . . . . . . . . 12

2.4 Differentiator using RC-like filter . . . . . . . . . . . . . . . . . 13

2.5 Frequency response of a differentiator using RC-like filter . . . . . . . . . . . 14

2.6 Grounded Capacitor and Clamped capacitor methodss . . . . . . . . . . . 15

2.7 Follower integrator circuit . . . . . . . . . . . . . . . . . . 15

2.8 The diffl circuit . . . . . . . . . . . . . . . . . . . . . . 16

2.9 The diff2 circuit . . . . . . . . . . . . . . . . . . . . . . 16

2.10 Frequency response of diff2 (simulated) . . . . . . . . . . . . . . . 17

2.11 Varying bias conditions versus Diff2 frequency resposne . . . . . . . . . . . . 18

2.12 Current output based differentiator . . . . . . . . . . . . . . . . . . . . . . . . . . . . . . . . . .

2.13 Basic idea for differentiation . . . . . . . . . . . . . . . . . . 19

2.14 Limiter circuit . . . . . . . . . . . . . . . . . . . . . . . . . 20

2.15 Frequency response of clamped capacitor based differentiator (simulated) . . 20

2.16 Feed-back and feed-forward differentiator models . . . . . . . . . . . . . . . . 21

2.17 The basic differentiator circuit . . . . . . . . . . . . . . . . . 22

2.18 The improvised differentiator circuit . . . . . . . . . . . . . . . . . . . . . . . . . . . . . . . . . . 23

2.19 The Tobi element . . . . . . . . . . . . . . . . . . . . 24

2.20 Layout of differentiator block . . . . . . . . . . . . . . . . . 24

2.21 Demonstration of Sine to Cosine conversion . . . . . . . . . . . . . . 26

2.22 Frequency response of differentiator circuit . . . . . . . . . . . . . . . . . . 27

2.23 Noise power in the output of differentiator circuit . . . . . . . . . . . . . . . 28

2.24 THD of the differentiator circuit . . . . . . . . . . . . . . . . 28

3.1 Basic idea for voltage-mode subtractor circuit . . . . . . . . . . . . . . . . 30

3.2 Subtractor circuit . . . . . . . . . . . . . . . . . . 32

3.3 Subtractor implentation . . . . . . . . . . . . . . . . . 34 
3.4 Layout of subtractor block . . . . . . . . . . . . . . . . . . 35

3.5 Voltage-mode subtraction of two sinusoidal signals . . . . . . . . . . . . . . . . . . . . . 36

3.6 Amplitude modulated signals through Subtractor circuit . . . . . . . . . . 37

3.7 Error vs. Amplitude for Subtractor circuit . . . . . . . . . . . . . . . . . 37

3.8 Output Error relation with input DC level . . . . . . . . . . . . . . . 38

3.9 Output Error relation with output DC level . . . . . . . . . . . . . . . . . . . . . . . . . . 38

3.10 Frequency response of subtractor circuit . . . . . . . . . . . . . . . . . . . . . . . . . . . . . . . . 39

3.11 THD of subtractor circuit. . . . . . . . . . . . . . . . . 39

3.12 Noise power in the output of the subtractor circuit . . . . . . . . . . . . 40

3.13 Relation between SNR and bias voltage . . . . . . . . . . . . . . . . . 40

4.1 Floating gate and Control gate . . . . . . . . . . . . . . . . 41

$4.2 \mathrm{I}-\mathrm{V}$ relation with charge present on the floating gate . . . . . . . . . . 42

4.3 Drain current relation with floating-gate charge . . . . . . . . . . . . . . 43

4.4 Electron injection . . . . . . . . . . . . . . . . . . . . . . . . . . . . . . . . . . . .

4.5 Electron tunneling . . . . . . . . . . . . . . . . . . . 45

4.6 Comparison of tunneling times for MOScap and Varactor tunneling junctions 46

4.7 Layout of MOSCAP based tunneling junction . . . . . . . . . . . . . . . . 47

4.8 Layout of varactor based tunneling junction . . . . . . . . . . . . . . . 47

$5.1 C_{4}$ frequency response. . . . . . . . . . . . . . . . . . . . 49

5.2 Response of the magnitude detector circuit (simulated) . . . . . . . . . . . 50

5.3 Transient response of differentiator circuit . . . . . . . . . . . . . . . . . 50

5.4 Transient response of subtractor circuit (simulated) . . . . . . . . . . . . . . . . 51

5.5 Single column of the analog front-end . . . . . . . . . . . . . . . . 51

5.6 Layout of the $0.18 \mu m$ auditory front-end . . . . . . . . . . . . . . . 52

6.1 Idea for hardware detection of sound events. . . . . . . . . . . . . . . . 54

6.2 Peak detector prototype with discrete components . . . . . . . . . . . . 55

6.3 Power consumption comparison in analog and digital systems . . . . . . . . . 56

6.4 Block diagram for the fabricated event detection system. . . . . . . . . . . . 57

6.5 Die shot of the event detection chip . . . . . . . . . . . . . . 58

B.1 Hysteretic differentiator circuit . . . . . . . . . . . . . . . . . 60

B.2 Different versions of hysteretic differentiator circuit . . . . . . . . . . . 62

B.3 The RL-type differentiator . . . . . . . . . . . . . . . . . 63 


\section{Chapter 1}

\section{Introduction}

Most circuits in contemporary VLSI systems are designed with digital technology as their basis. But computations, such as addition, subtraction, multiplication, differentiation and integration, are more suitable for analog circuits at a macroscopic level [1]. For example, a high quality microphone deployed to pick up sound from a musical concert generates a signal whose amplitude may vary from few microvolts to hundreds of microvolts. For extensive sound processing, all such signals go through significant processing using a digital signal processor (DSP). But these signals almost always are either too small for digitization or have unwanted out-of-band signals and noise associated with them. Due to this, the introduction of a modified analog front-end could enhance the signal quality. Also, these front-end operations can be performed using only a few transistors [2]. Along with the advantage of low-power consumption of circuits that operate in sub-threshold or weak inversion regions, analog systems can also describe naturally occurring systems more closely than the digital systems.

With progress in semiconductor research leading to smaller chip sizes and the increased use of small and mobile electronic devices, low-power systems have gained importance. Most modern electronic devices require significant processing capability to support the various applications they are used for, hence, leading to higher power consumption. Using analog systems operating in weak inversion region as a front-end for tasks like speech-recognition or environment sensing would greatly reduce the power needs of the devices. Mainly, these kind of devices would be useful in hearing aids, audio analysis and situations like remote 
monitoring where human presence needs to be avoided or would not be feasible. The main idea behind this kind of speech processing system is to closely replicate the signals produced by a human brain while processing auditory information it receives through the cochlea. So for the device to perform like human to perceive the environment, designing systems that mimic the human biology would be logical. Moreover, biology provides examples of very efficient perception systems, so trying to replicate, or at least come close, would present us with very efficient systems [3].

The objective of this overreaching project is to develop a speech-processing front-end, which can process auditory information, extract certain application specific features, and reduce the burden on the next level of digital processing systems.

\section{$1.1 \quad$ Analog Front-ends}

In a typical real-time system, the transducer produces current or voltage signals depending on the changes in its environment. These signals are mostly in varying time intervals and varying magnitude depending on the input the transducer receives. The output signals from the transducers are later processed by digital signal processors (DSPs) after being converted to digital signals using an analog to digital converter (ADC). A lot of computational power would be required to process all the data that the transducers dump onto the DSP. Using a few custom-built circuits to properly decompose the raw data from the transducer would greatly reduce the burden on the power-hungry DSPs (Fig. 1.1).

The focus of this project is to design such custom-built circuits for band-pass filtering of speech signals while adding specific computational capability to the circuits. The main aim here is to produce a few compact circuits with low-power consumption, allowing highlyparallel systems used in real-time data collection and processing. For this particular project, a large number of computations are done on raw data normally by the digital end. This could easily be done by an analog system (Fig. 1.3). Also, this analog system will have continous-time circuits for real-time operation. This new model could lead to lower power consumption as the transistors are biased in sub-threshold and also they could be more efficient [3]. 


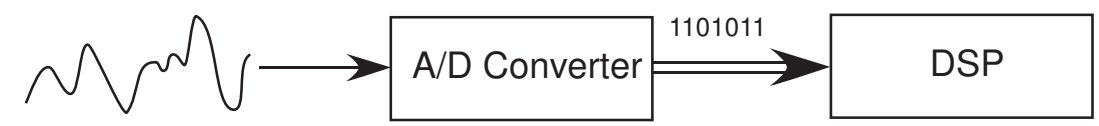

(a)

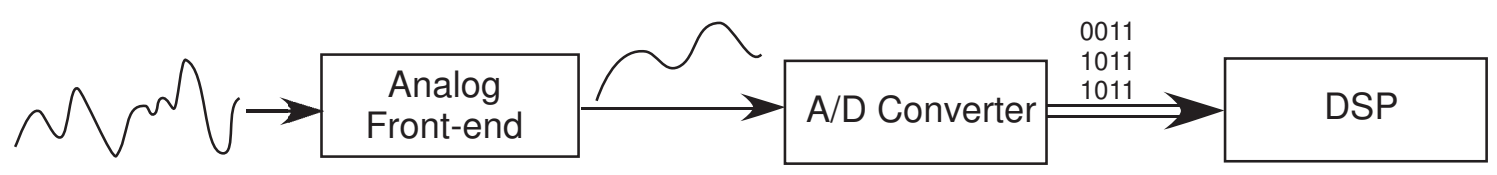

(b)

Figure 1.1: (a) More data needs to be processed when raw information is sent to the DSP (b) Adding analog front-end does initial processing and reduces burden on the DSP

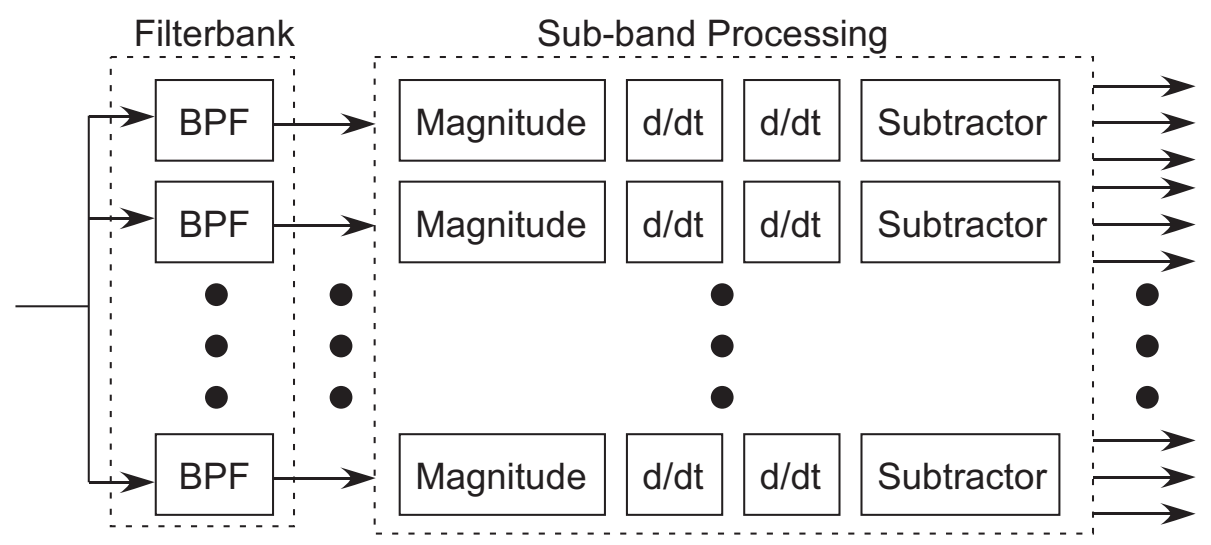

Figure 1.2: Block diagram of the analog front-end

The design of the complete system was done in two parts as illustrated in Figure 1.2. The first part of the design deals with filtering the input signals into sub-bands and performing magnitude detection on the sub-banded signals. The second part, which is the core of the research work described in this thesis, deals with the design of analog derivative and subtractor circuits. These blocks are interconnected using multiplexing circuits. Therefore they can be connected in various ways to cater to the needs of different algorithms. These functional blocks are designed to accommodate post-fabrication programmability, which can be done by using the floating-gate structures. The next few sub-sections give an overview of the functional blocks designed asa part of this project for sub-band processing in the analog front-end. 


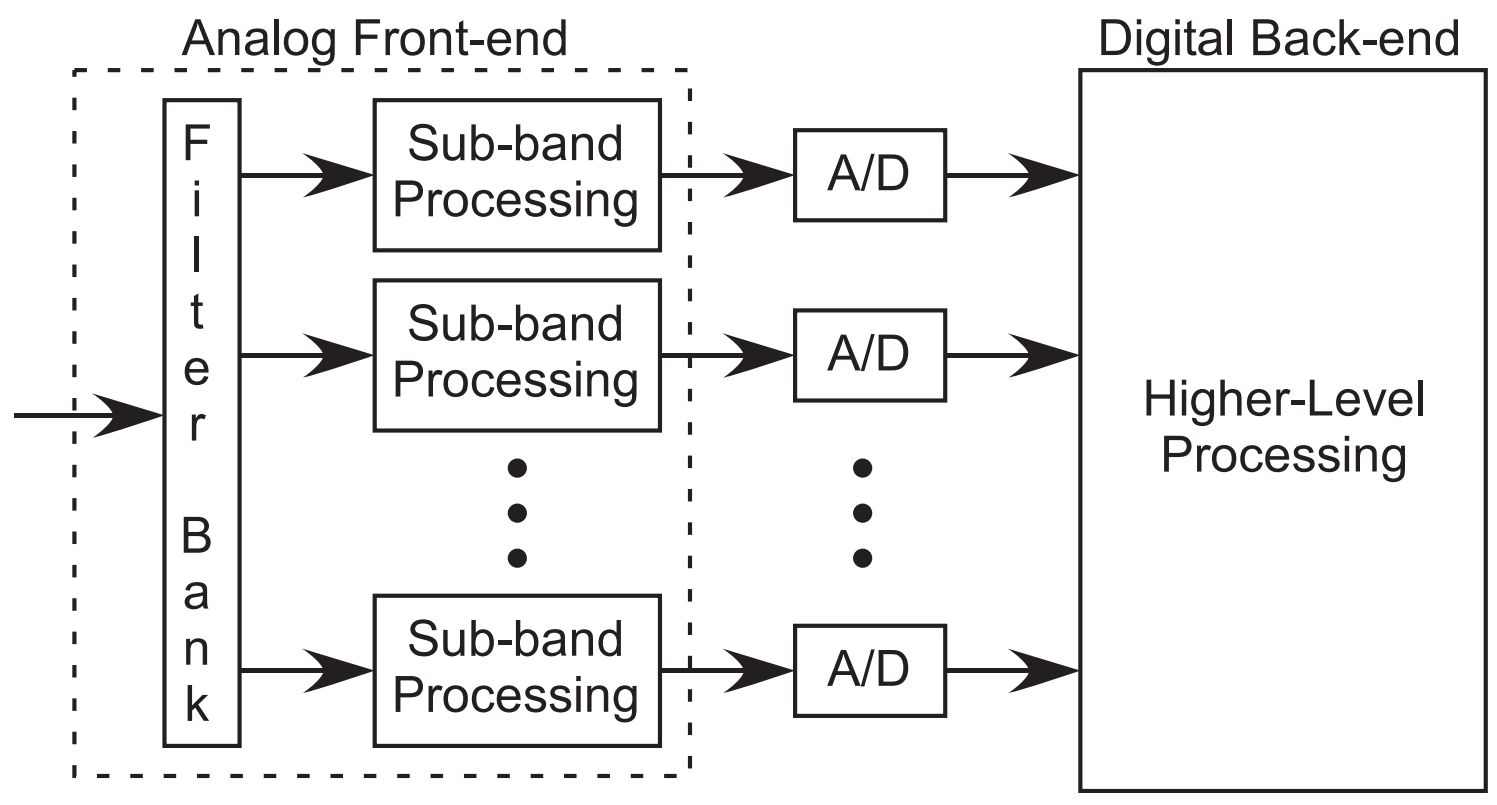

Figure 1.3: A real-time system with analog front-end.

\subsubsection{Differentiator}

A differentiator circuit has been built as part of the analog front-end, to aid in signal processing after spectral decomposition of the input. This block is required to implement algorithms which require information about temporal changes in the input signals. The circuit is based on the clamped-capacitor approach to obtain the derivative output. It uses only MOSFETs (metal-oxide-semiconductor field-effect transistors) in different combinations to emulate a capacitor, a resistor and a high gain amplifier, resulting in a very compact circuit.

The circuit is designed with post-fabrication tunability and can either work in low-power, low signal-quality mode or high-power and high signal-quality mode. This can perform differentiation of signals over the entire audio range, from $20 \mathrm{~Hz}$ to $20 \mathrm{KHz}$. Also, the gain of the circuit is adjustable and thus can work at various signal levels. This consumes just $\mu W s$ of power and also can be programmed to have different gain levels. 


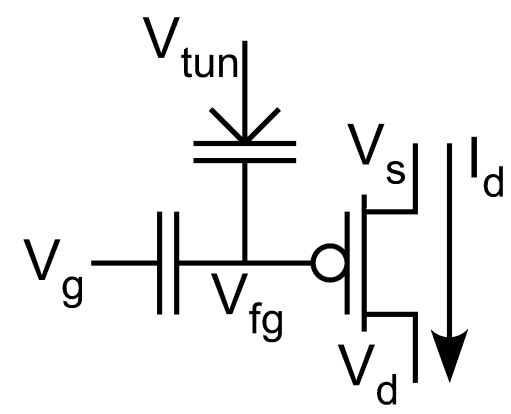

Figure 1.4: Schematic of a floating-gate transistor

\subsubsection{Subtractor}

The subtractor block is used in post-processing of the band-pass filtered signals. This block is connected to other blocks of the analog front-end system using multiplexing circuits to suit various post-processing combinations. The main function of this block is to perform voltage-mode subtraction using very low-power and produce high quality signals. It is designed based on the circuit presented in [4]. It works in the entire audio range, from $20 \mathrm{~Hz}$ to $20 \mathrm{KHz}$ and has a dynamic range of $64 \mathrm{~dB}$ and power consumption is in $\mu W s$. Like the differentiator circuit, even this uses only MOSFETs, leading to a very compact structure. Moreover, the basic subtractor circuit requires just a single constant bias for its operation over the entire audio range, making it very easy to use. Although designed to be used for subtraction of sub-banded audio signals, this can also be used for addition also.

\subsubsection{Floating-gate Transistors}

To easily program various functional blocks of the analog front-end after fabrication, floating-gate MOSFET arrays are used. Most non-volatile solid-state memory devices use Floating-gate MOSFETs (Fig. 1.4) as the basic building blocks [5]. In these kind of MOSFETs, the gate is electrically isolated as it is surrounded by insulator ( $\mathrm{SiO} 2)$ on all sides. This helps in maintaining the charge on the gate as a constant for long periods of time. Methods like Hot-Electron Injection [6] or Tunneling [7] are normally used to alter the charge on the floating-gates. 
As the gate of these transistors does not have any physical contact with other conductors on the chip, the capacitively induced charge can be stored and used to set the bias for other devices. The floating-gate MOSFETs can be used to precisely set the bias for operating the systems reliably and efficiently. This gives the ability to program various functional blocks, depending on the application. By suitably designing a matrix of these basic floating-gate MOSFETs, the total analog front-end can be programed easily and efficiently. Also, this helps to offset any mismatches during fabrication of the chips and leads to better quality systems.

\subsubsection{Filter-Bank with Sub-band Processing}

The complete analog front-end comprises the functional blocks for spectral decomposition, magnitude detection, differentiation and subtraction. The complete system includes an array of 16 channels of each of the analog front-ends. Each channel is used for sub-banding and processing the audio signals of a particular frequency range within the audio range. The total system was fabricated on a $0.18 \mu \mathrm{m}$ CMOS (complementary MOS) process and was designed to have high flexibility of connections between each block post-fabrication. This would allow to perform various applications depending on the requirement. Along with this, one more filter-bank with reduced functionality and only 8 channels was used to design an intelligent wireless analog event-detection system. This was done on a $0.5 \mu \mathrm{m}$ CMOS process. These front-ends could be used in the future for applications like speech-recognition, audio analysis or hardware-based event detection.

\subsection{Basic Building Blocks}

The main focus of this project was to build a low-power system, while having good performance and signal quality. This system is intended to compliment the DSP, and help reduce the burden on it, in turn, leading to lower power consumption. CMOS linear devices and digital devices use the same fabrication process so the analog and digital systems can be integrated onto a single chip, making the production easier and cheaper. Also, as the range of applications performed by a chip increases, it would require both analog and digital 


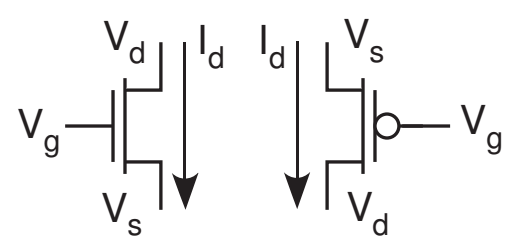

Figure 1.5: Symbols for an n-channel (left) and p-channel (right) MOSFETs

circuits. This calls for using the same process (CMOS) for designing both types of circuits leading to cost-effective fabrication and packaging. Therefore in the future, to accommodate both digital and analog systems on a single chip, CMOS transistors (Fig. 1.5) were used as the basic building blocks of the analog front-end in this thesis. The use of analog ciruits also helps to reduce power consumption because CMOS devices dissipate power only during the switching of the gates [1]. For analog purposes, most of the transistors are biased in the sub-threshold region leading to a low overall-power consumption.

The current through an nFET transistor in this region is given by:

$$
I=I_{0} e^{\frac{\kappa V_{g}}{U_{T}}}\left(e^{-\frac{V_{s}}{U_{T}}}-e^{-\frac{V_{d}}{U_{T}}}\right)
$$

where $\kappa$ represents sub-threshold slope factor, $U_{T}$ represents thermal voltage and $I_{0}$ is the current which varies with the size and fabrication process of the transistor $[3,8]$. The operating current is normally less than $1 \mu A$. This helps in designing low-power systems which consume far less power compared to the digital systems.

\subsection{Organization}

This document is divided into 6 chapters. Chapter 2 details the various models considered for the derivative circuit and the design procedure of the final derivative circuit. This also includes design considerations and the final post-fabrication results from the circuits built using $0.18 \mu m$ CMOS process. Chapter 3 describes the Subtractor block of the analog frontend. The design procedure of the Subtractor circuit, the techniques to bias it and the results obtained from the post-fabrication testing of the design in $0.18 \mu \mathrm{m}$ CMOS process are shown. Chapter 4 explains about the floating-gate transistors and the methods to program 
and erase charge from the floating-gate. Chapter 5 deals with the overall function of the analog front-end as a whole. The flow of signals between various blocks of the analog frontend is discussed and explains how each block would fit in. It also gives the details of how the floating gate structures were used to program various blocks of the analog front-end. Chapter 6 discusses the application of the analog systems in wireless sensor networks to reduce power conusmption and future scope of this work.

All the data presented in this thesis is from circuits fabricated in either $0.5 \mu \mathrm{m}$ or $0.18 \mu \mathrm{m}$ CMOS process unless mentioned as simulated. 


\section{Chapter 2}

\section{Derivative Circuit}

The derivative circuit designed as part of this project is used to implement various speech processing algorithms on the audio signals after they have been spectrally decomposed. That is, only the sub-banded signals would be fed as input to the derivative circuit. The circuit helps in extracting the temporal change information from various band-passed signals in the audio frequency range. To obtain the derivative of a sub-banded signal, the gain must be unity at the particular frequency of operation and the phase shift must be $90^{\circ}$. For example, if a sine wave with $100 \mathrm{mV}$ amplitude and $1 \mathrm{kHz}$ frequency is the input, ideally the output should be a cosine with the same amplitude and frequency (Fig. 2.1). To have such a response, the ideal frequency response should have a "derivative slope" of $20 \mathrm{~dB} / \mathrm{dec}$ and a "derivative phase shift" of $90^{\circ}$ at the frequency of operation as shown in Figure 2.2. It is important that the two conditions are satisfied at the operating frequency for differentiation to happen. Also, as the phase shift starts to drop as we go closer to the corner frequency, the operating frequency must be $\leq 0.1$ times the corner frequency to obtain a derivative response.

Ideally the derivative circuit should have a transfer function as

$$
f(t)=A \frac{d(x(t))}{d t}
$$

where $\mathrm{A}$ is the gain of the circuit.

In this project, the main requirements were to have low-power consumption (in the $\mu W$ range) and moderate signal to noise ratio. As the input is always a spectrally decomposed 


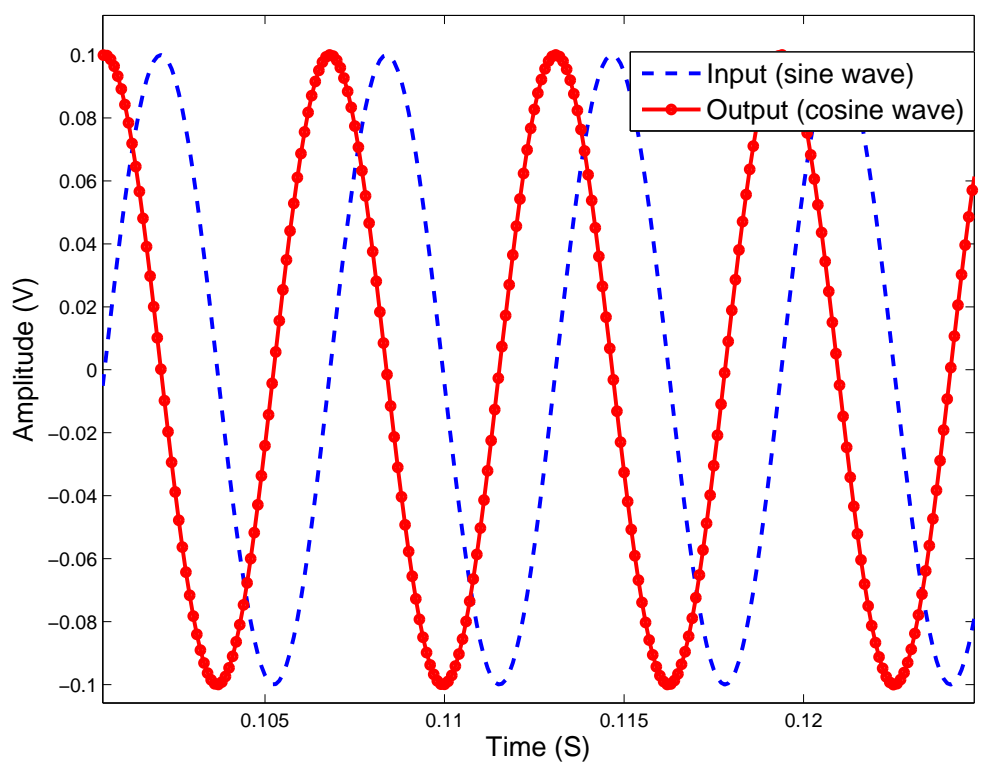

Figure 2.1: Differentiation of a sine wave (simulated)

signal, the circuit should be designed so that the frequency at which the unity gain occurs can be tuned post-fabrication. Also, the operating frequency (within the audio range of $20 \mathrm{~Hz}$ to $20 \mathrm{kHz}$ ) should be adjustable based on the frequency of the input signal. Moreover, we would also like to compress really large changes in the input, so that the output does not get clamped at the supply rails. With these specifications and goals many models were considered to perform this function and to work within the specified power and performance range. This chapter describes the design and operation of the final differentiator circuit.

\subsection{Different Kinds of Derivative Models}

The following sub-sections describe the various kinds of derivative circuits that were considered for building the required derivative functional block for this project.

\subsubsection{First-Order High-Pass Filter}

The frequency response from a first-order high-pass filter resembles that of a derivative circuit using analog circuits. A simple RC-like high-pass filter is designed using an opera- 


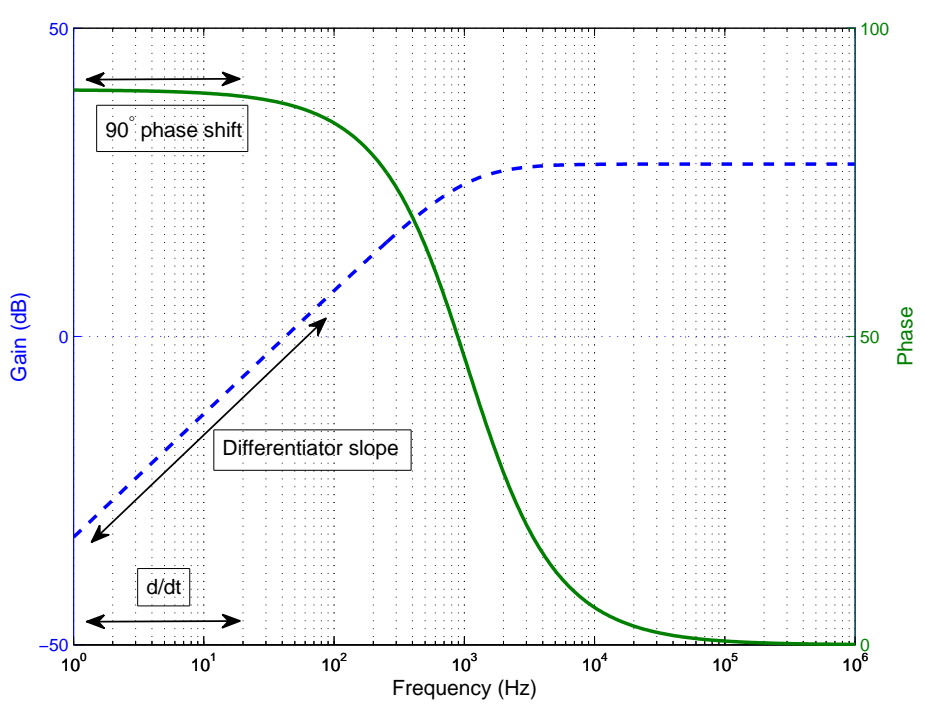

Figure 2.2: Ideal frequency response of a differentiator (simulated)

tional transconductance amplifier as the active resistor (Fig. 2.4). This can be used to tune the circuit for operation at different frequencies. In theory, it would give frequency response as shown in Figure 2.5. This creates the required "differentiator slope"; a $20 \mathrm{~dB} /$ decade slope and $90^{\circ}$ phase shift at frequencies approximately 0.1 times the corner frequency, $f_{c}$. The corner frequency can be tuned easily by changing the transconductance of the Operational Transconductance Amplifier (OTA). In theory, the gain at frequencies above the corner frequency would be close to 1 . But in practice, for gain to be 1, a large capacitor is needed (about $10 \mathrm{pF)} \mathrm{(Fig.} \mathrm{2.3),} \mathrm{which} \mathrm{would} \mathrm{lead} \mathrm{to} \mathrm{real} \mathrm{estate} \mathrm{problems} \mathrm{on} \mathrm{the} \mathrm{chip.} \mathrm{This} \mathrm{is} \mathrm{due}$ to the higher coupling provided by large capacitors, leading to higher gain. As a test case for this circuit, a sinusoidal signal is given as the input and for perfect differentiation, the output would also have to be a sinusoidal signal but $90^{\circ}$ out of phase compared to input, that is,

$$
\frac{d}{d t}(\sin (t))=\cos (t)
$$

As can be deduced from Figure 2.5, the input signal should have a frequency less than 0.1 times the corner frequency, for obtaining a differentiated output. The positive features of this model are that it is a simple and compact circuit, provides a differentiator slope and differentiator phase. But on the other side, the negative features about this circuit are that 


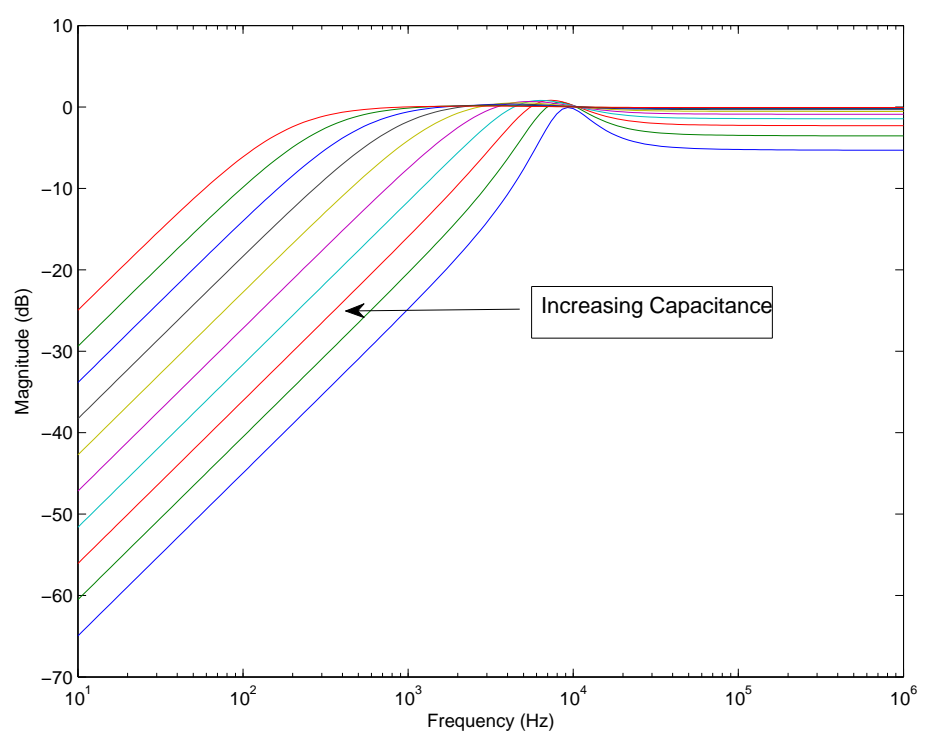

Figure 2.3: Variation of gain with size of the capacitor. Increasing the capacitance moves the frequency respone laterally thereby increasing the gain at the required frequency (simulated)

there is a significant attenuation of the derivative signals and the SNR is extremely low due to very small signal at the output. A gain stage (a simple common-source amplifier) was used to increase the gain, but the Signal-to-Noise Ratio (SNR) was not improved considerably and it also introduced high Total Harmonic Distortion (THD). Even increasing the size of the capacitor ( $C 1$ in Fig. 2.4) has also not sufficiently improved the performance of the circuit. Therefore, this would not be a perfect fit for the desired specifications.

\subsubsection{Capacitor for Derivative}

This model is based on the relation between current and voltage applied on a capacitor. The transfer function of a capacitor is,

$$
I_{\text {out }}=C \frac{d}{d t}\left(V_{\text {in }}(t)-V_{\text {out }}(t)\right)
$$

The current through a capacitor represents the derivative of the voltage across it. Therefore, by measuring the current, the derivative of the input voltage signal can be found. There are two major methods of creating a derivative circuit based on this approach, the grounded capacitor approach and the clamped capacitor approach as shown in Figure 2.6. By using 


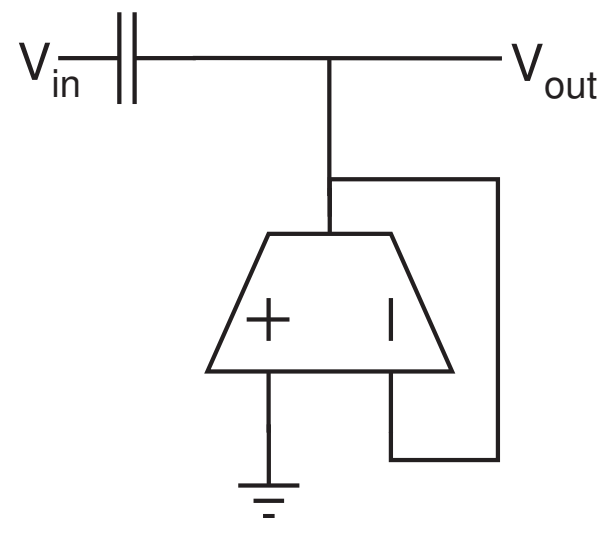

Figure 2.4: Differentiator using RC-like filter

the transfer function of a capacitor, it can be deduced that the derivative can be obtained by taking the difference of a signal from a slightly delayed version of itself. A close approximation of this time domain derivative can be obtained by comparing the input signal with its timeaveraged version. One way of achieving this is subtracting the low-pass version of the signal from the original one.

An effective way of implementing this in VLSI would be to use a follower-integrator (Fig. 2.7) and subtract the original version of the signal from the output of the follower-integrator. The subtraction can be easily done by a trasnconductance amplifier which has the transfer function as, $V_{\text {out }}=A\left(V_{\text {in+ }}-V_{\text {in- }}\right)$. This implementation is illustrated in 2.8 and is known as Diff1 circuit [3]. But the problem with this circuit is that it has an open loop configuration and even a small change in the offset voltage can lead to very high gain and clamp the output at the supply rails.

A different model of the circuit based on the same concept is considered, which has a negative feedback configuration. Also, this limits the huge changes in the output due to mismatched offsets or mismatches caused during fabrication. This alternative circuit is known as Diff2 (Fig. 2.9) [3]. In this circuit, the amplifier $A 2$ (Fig. 2.9) is in a follower configuration and its output is given to the negative input of the amplifier $A 1$ (Fig. 2.9). This performs the subtraction of the the time-averaged signal from the original version. The 


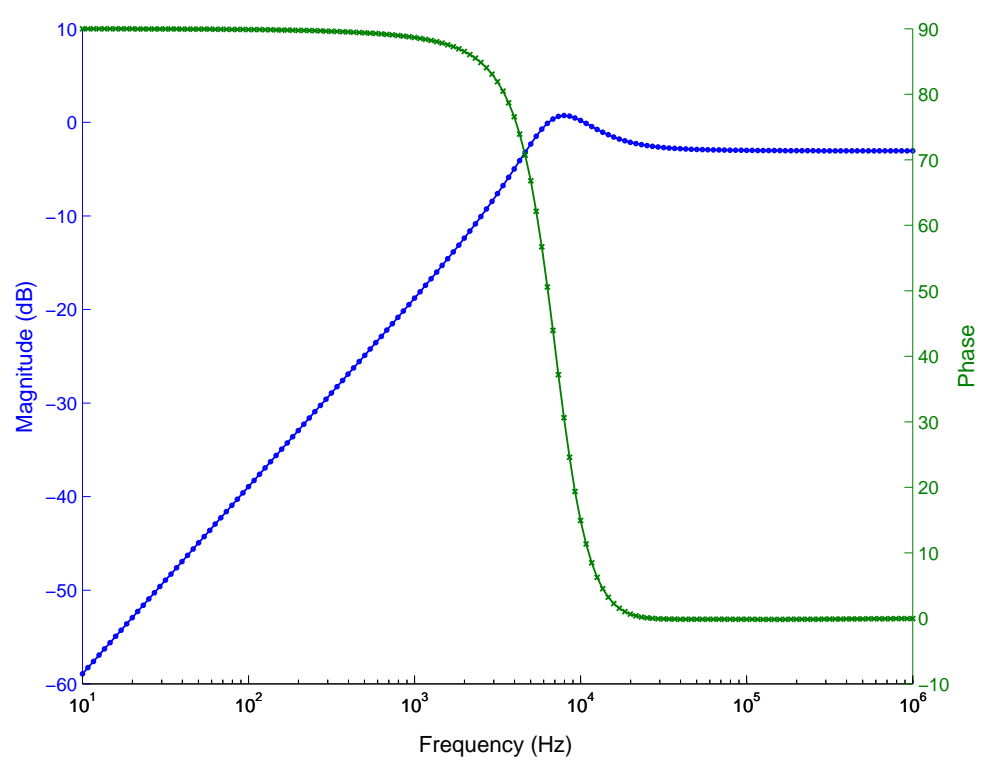

Figure 2.5: Frequency response of a differentiator using RC-like filter

diff2 can be characterized by the following equations:

$$
\begin{gathered}
V_{\text {out }}=A\left(V_{\text {in }}-V_{c}\right) \\
V_{c}=\frac{1}{1+\tau s} V_{\text {out }}
\end{gathered}
$$

where $\mathrm{A}$ is the open circuit voltage gain of $A 1$ and $\tau=C / G$, with $\mathrm{G}$ being the transconductance of A2. With these equations, we can obtain the transfer function of Diff2 as,

$$
\frac{V_{\text {out }}}{V_{\text {in }}}=\frac{A}{(A+1)} \frac{1+\tau s}{(1+(\tau /(A+1)) s)}
$$

It can be deduced from the transfer function that, at low frequencies, this circuit acts as a unity-gain follower. At intermediate frequencies, the circuit behaves like a differentiator, and at high-frequencies it acts as an amplifier. But this transfer function does not take into account, the effect of the parasitic capacitances. These issues surface in the physical implementation of the circuit. Therefore, when implemented in a physical setup, the frequency response of the circuit does not correspond to the theoretically derived conclusion. Instead, the response is as shown in Figure 2.10 and 2.11. The output peaks at high frequencies, caused by the parasitic capacitance at the output node, $V_{\text {out }}$. 


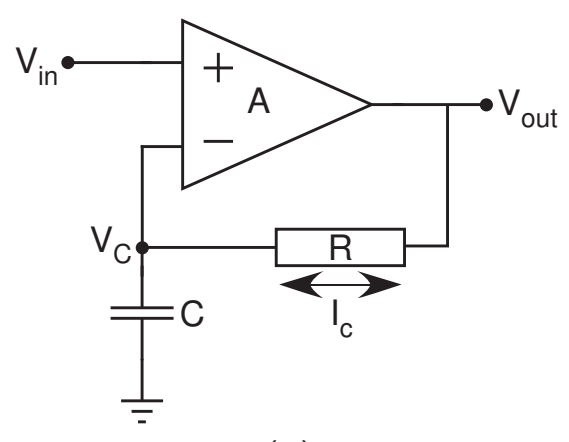

(a)

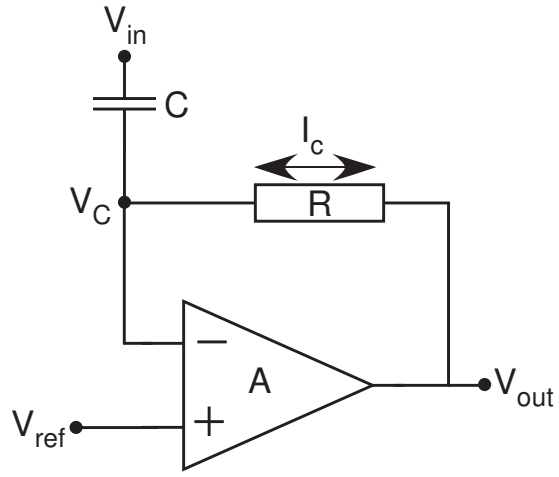

(b)

Figure 2.6: Grounded Capacitor and Clamped capacitor approaches for differentiation

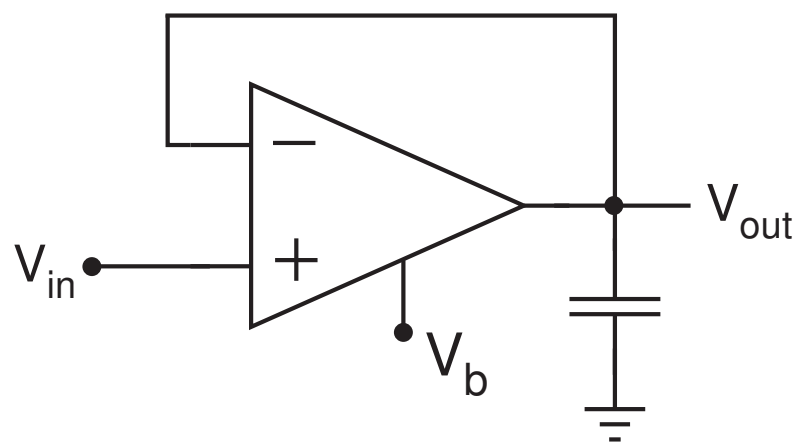

Figure 2.7: Follower integrator circuit. The bias voltage of the transconductance amplifier can be used to modify the bias integrator's time constant.

When this model is considered in the perspective of the requirements for the current project, it does not fit in perfectly. First, it has the "differentiator slope" for a small range of frequencies. It also has very high gain. Second, with a high gain, it would result in higher THD as the higher harmonics would be amplified exponentially. Higher noise levels are also observed in this circuit. Also, it causes clipping and distortion at high frequencies as the high gain can cause the output to go rail-to-rail. Apart from all these factors, the output might even oscillate due to high-frequency Q. Therefore, this model also is not suitable for the required application. 


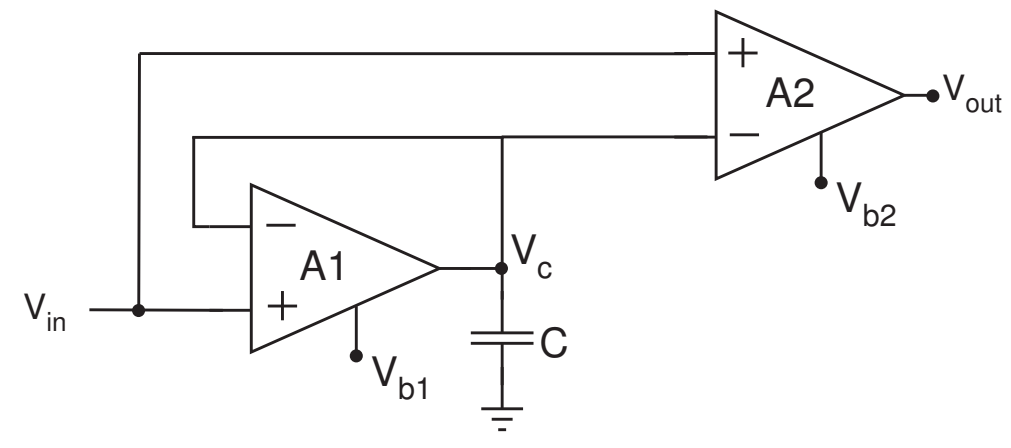

Figure 2.8: The diff1 circuit

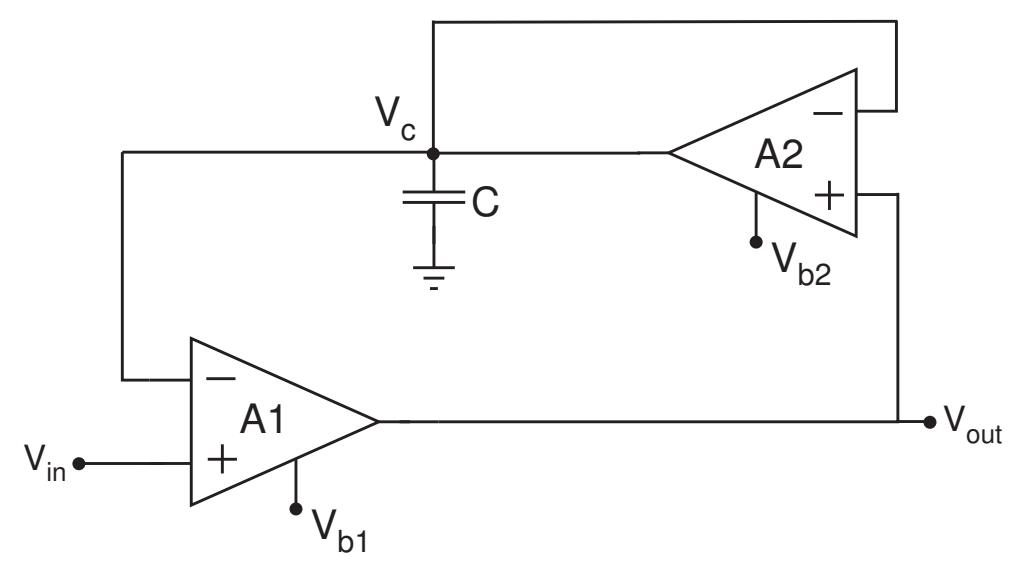

Figure 2.9: The diff2 circuit

\subsubsection{Current-Output Derivative Circuits}

This is yet another class of derivative circuits based on using current-output. This extends the linearity feature used in previous differentiator models described here by using the current outputs (Fig. 2.12). This circuit is essentially a Capacitively-Coupled Current Conveyor $\left(\mathrm{C}^{4}\right)[9]$ circuit along with a symmetric source follower with current outputs. Even when the voltage input signals are distorted, the current is linear. The output currents are typically the rectified values, and this works only for signals with a large amplitude. This is caused by the 'Nulls' in the symmetric source follower circuit. Also, leakage current in this circuit would be a significant issue when operating with very small signals. This circuit is not a suitable fit for this project as the focus here is to design a circuit which could be used in the total audio range and also have a unity gain derivative output. 


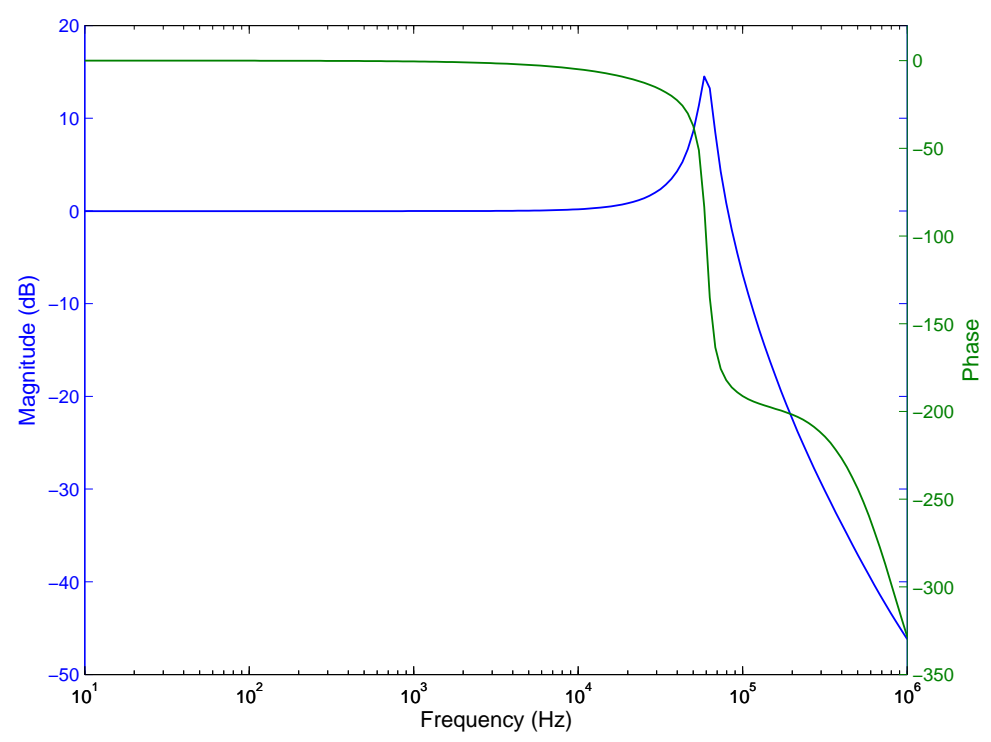

Figure 2.10: Frequency response of diff2 (simulated)

\subsubsection{Differently-Clamped-Capacitor based Differentiator}

The clamped capacitor based approach for the differentiator circuit did not yield the required results for this project. Instead, a variation of the clamped-capacitor based derivative circuit was considered. This circuit has a capacitive input, a high-gain inverter and a follower connected OTA feedback to auto-bias the inverter connected as shown in Figure 2.13 [10]. It also has a charge limiting circuit (built using only two transistors) (Fig. 2.14), in the feedback path to limit the OTA from being saturated. The total setup is essentially a Capacitively-Coupled Current Conveyor $\left(\mathrm{C}^{4}\right)$ with a feedback resistor.

This circuit gives the differentiator output, and to bump up the gain at the output stage, it has an extra high-gain inverter. This results in having a pass band gain of unity, which is an essential feature for this project. Figure 2.15 shows the frequency response and phase response of the circuit. With proper bias conditions, this circuit can give a differentiator output. The total harmonic distortion (THD) is comparatively lower but the SNR is only $20-30 \mathrm{~dB}$. Also, the output DC level does not stay put at a particular level, but changes with the bias conditions. That is, when the circuit bias is changed based on input signal frequency, even the output DC level shifts. Therefore an extra level-shifting circuit is needed 


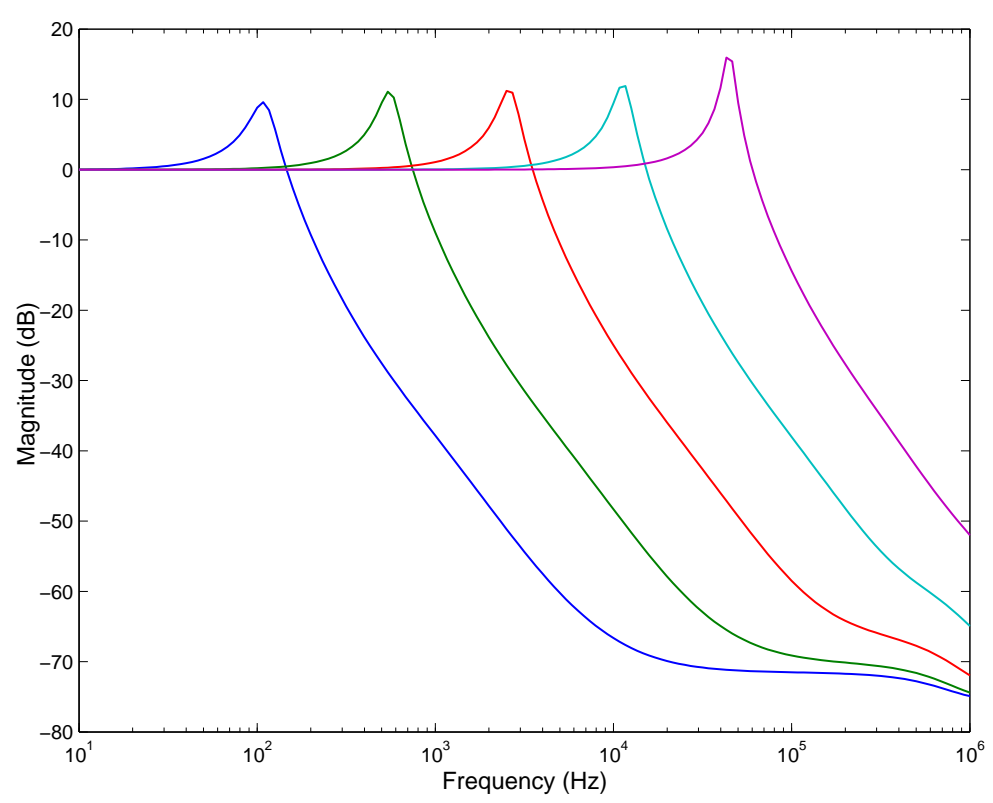

Figure 2.11: Frequency response of diff2 for varying bias conditions. None of these provide the "differentiator slope" (simulated).

to control the DC level of the output. With slight modifications, this circuit can be used for the application in this project.

\subsection{Design of Differentiator Circuit}

A simple RC-like high-pass filter can be used for obtaining the derivative slope and the $90^{\circ}$ phase shift. A circuit designed using an operational transconductance amplifier as the active resistor (Fig. 2.4) has the frequency response as shown in Figure 2.5. The problem with this circuit is that the frequencies at which the derivative slope and phase occur have a very low-gain. As stated earlier, the optimum operating frequency should be approximately 0.1 times below corner frequency, and in this case the gain is very-low at such frequencies leading to a very low SNR.

Based on the various circuits for obtaining a differentiator output (described in Section 2.1), it has been observed that none of them completely suit the specifications required for this project. Of all the circuits, the circuit using the high-gain inverter and capacitively coupled input (as described in Section 2.1.4) is the one which comes close to fulfilling the 


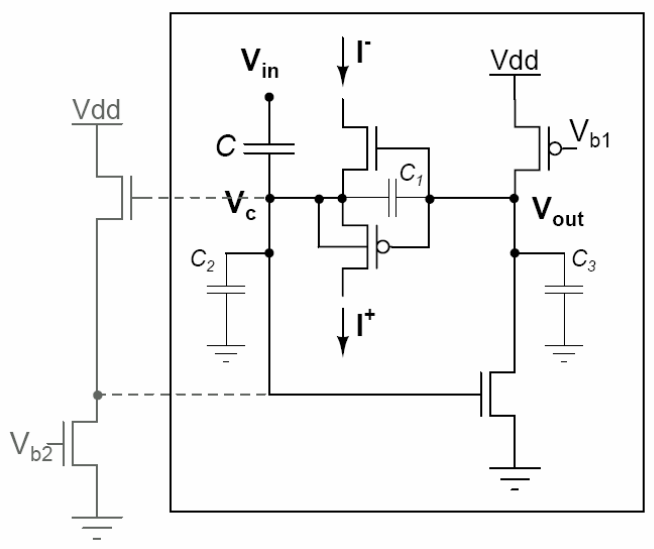

Figure 2.12: Current output based differentiator

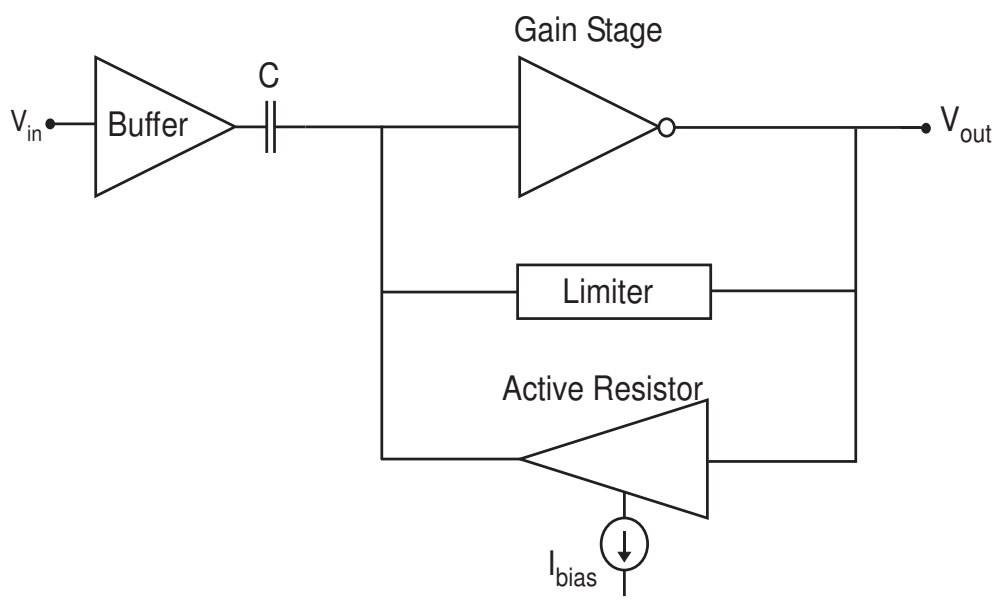

Figure 2.13: Basic idea for differentiation

specifications for this project. There have been two major issues with the circuits described in Section 2.1. If the output has low-gain for the desired frequency, then the power consumption is low and THD is decent but SNR is very low. And when the gain is high for desired frequency, more power is consumed, THD is very high and SNR is within tolerance levels.

To overcome these problems, the differently clamped-capacitor circuit has been modified to suit the specifications of this project. With this approach, there are two choices: either a feed-forward design or a feedback design. Feedback methods are more suitable in this case, as they decrease the requirement of matching the elements precisely. Also, this being 


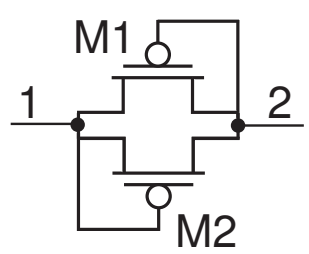

Figure 2.14: Charge limiter circuit using only two transistors

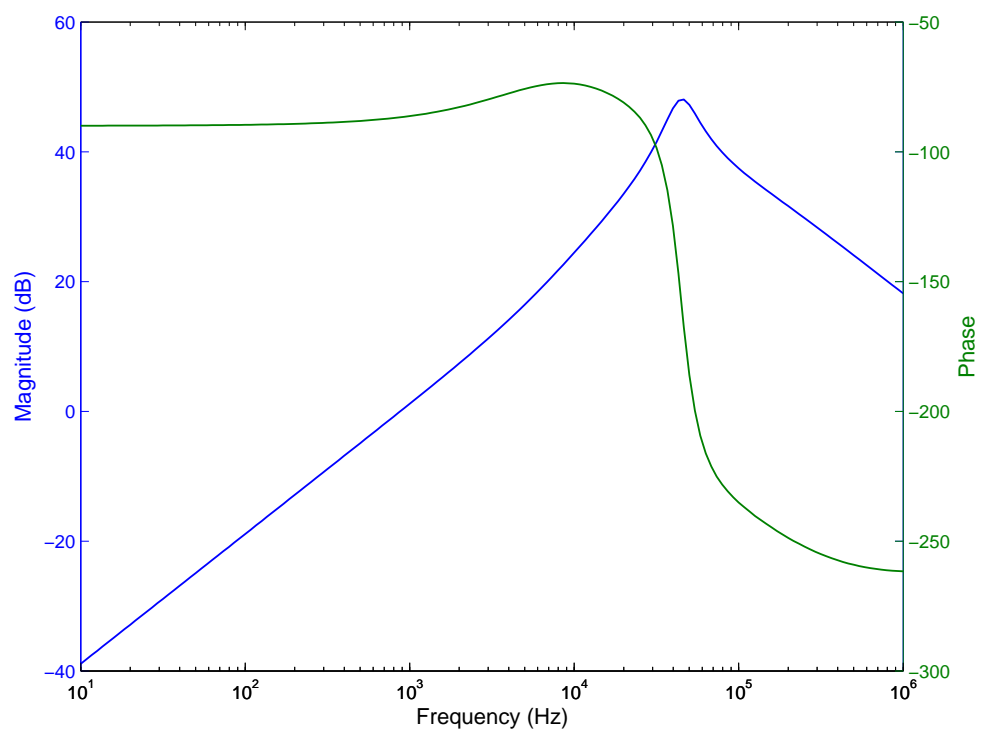

Figure 2.15: Frequency response of clamped capacitor based differentiator (simulated)

a relatively new CMOS process for design of analog circuits, it would be useful to eliminate such cases of mismatch wherever possible. There are three feasible architectures for obtaining the differentiated output. The configuration in Figure 2.16(a) is a feed-forward differentiator whose transfer function is

$$
H(s)=\frac{A R C s}{1+R C s} .
$$

Here, $R C$ gives the time constant of the circuit and $\mathrm{A}$ is the gain. If, $R C s \ll 1$, then from Eqn. 2.7,

$$
H(s) \approx R C s .
$$

Similarly, for the configuration in Figure 2.16(b), the transfer function is

$$
H(s)=\frac{-A R C s}{1+A+R C s} .
$$



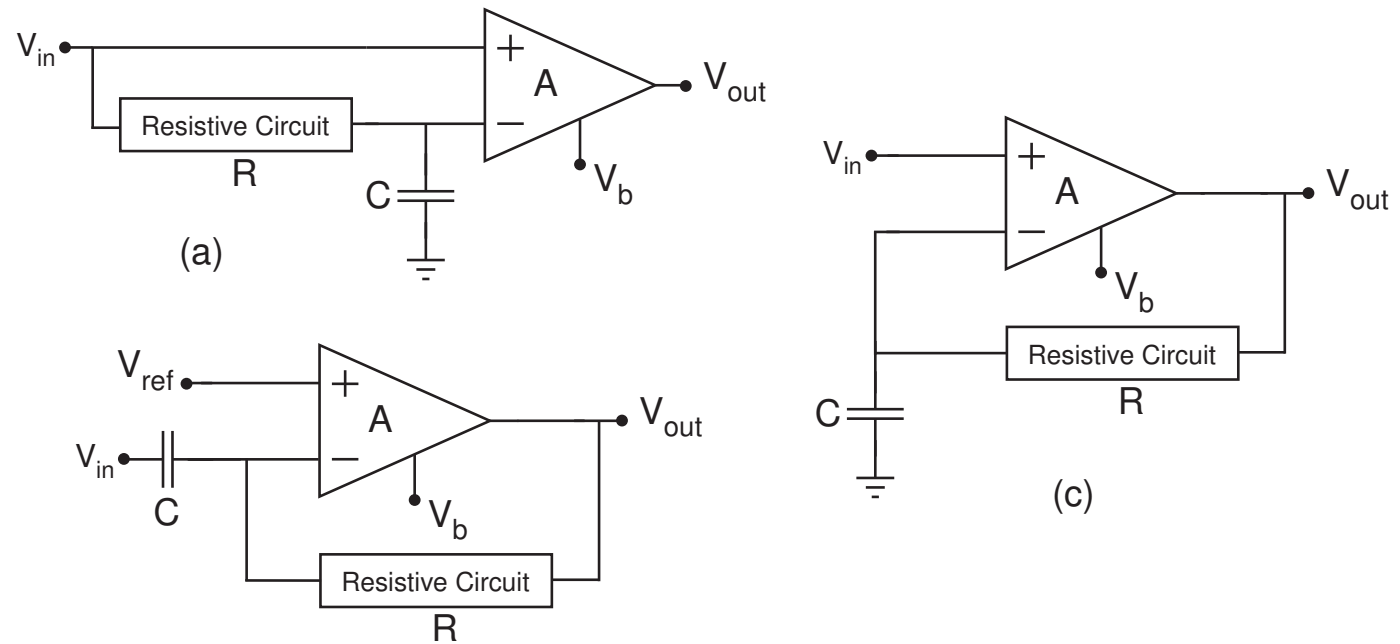

(b)

Figure 2.16: (a) Model for differentiator circuit with feed-forward and RC delay line. (b) Feed-back differentiator with inverter as a high-gain element.(c) Another feed-back model with an RC-integrator in the feed-back loop

If the gain $A \gg 1$, then from Eqn. 2.9,

$$
H(s) \approx-R C s
$$

and for the configuration in Figure 2.16(c), the transfer function is

$$
H(s)=\frac{A(1+R C s)}{1+A+R C s} .
$$

If the gain $A \gg 1$, then from Eqn 2.11,

$$
H(s) \approx 1+R C s
$$

The use of the third configuration Figure 2.16(c) would make it complicated to bias the circuit and put limitations on the inputs as the output steady-state voltage is dependent on the input current level.

In the configuration shown in Figure 2.16(b), the output steady state voltage is dependent on the DC characteristics of the inverting amplifier. The inverting amplifier can be automatically biased by an active resistor circuit in the feedback loop. The resistive circuit limits the lowest frequency at which the differentiator would function normally. In standard processes, passive resistors have low resistivity in the context of the requirements of this 


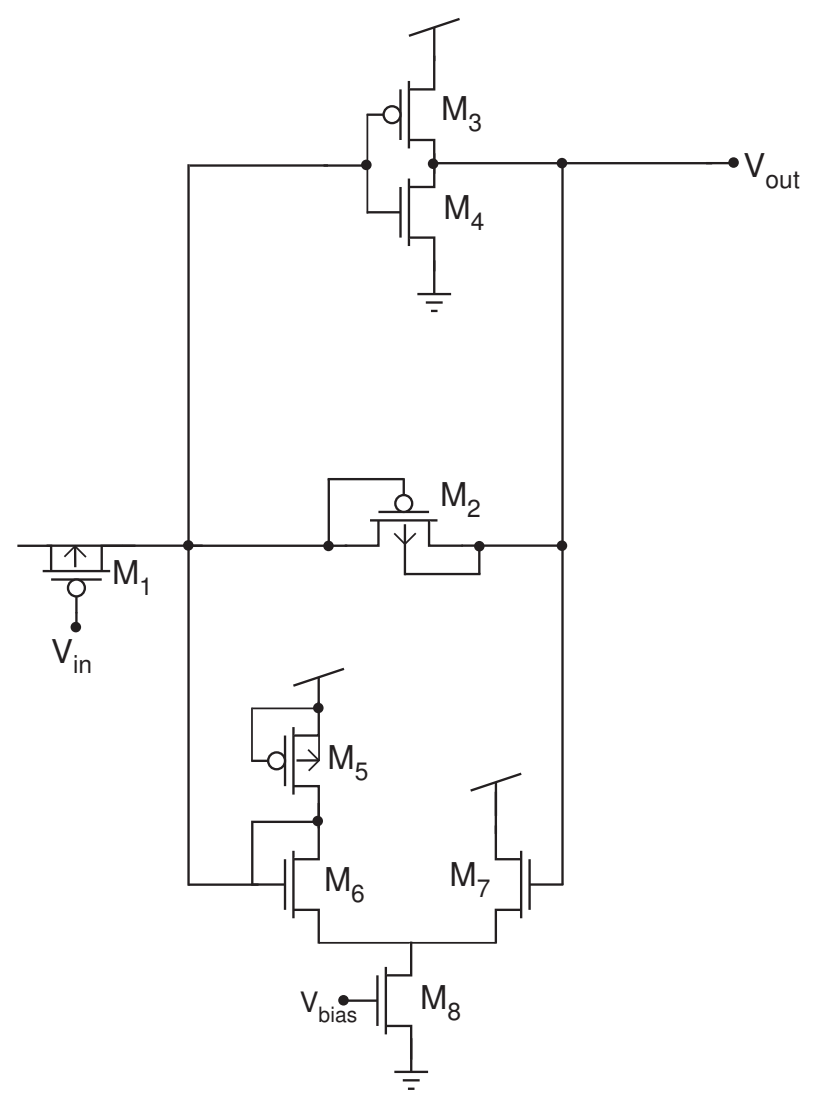

Figure 2.17: The basic differentiator circuit

circuit. The active resistors can be designed using a modified version of an Operational Transconductance Amplifier (OTA). The active resistor may go into saturation if the charge is too high and result in non-ideal output. Therefore, a limiting circuit is used to limit the charge on the active resistor and preventing it from saturating. The limiting circuit can inject a large amount of charge onto the output node of the active resistor. The amount of charge injected depends on the limiting circuit. This way, a differentiator circuit can be designed to meet the specific requirements of this project. The following sub-section describes each block of this circuit.

\subsubsection{Various Blocks of the Differentiator Circuit}

The differentiator circuit designed here is shown in Figure 2.17. As can be seen from the functional block diagram of the differentiator circuit, the input is AC coupled. Here, a 


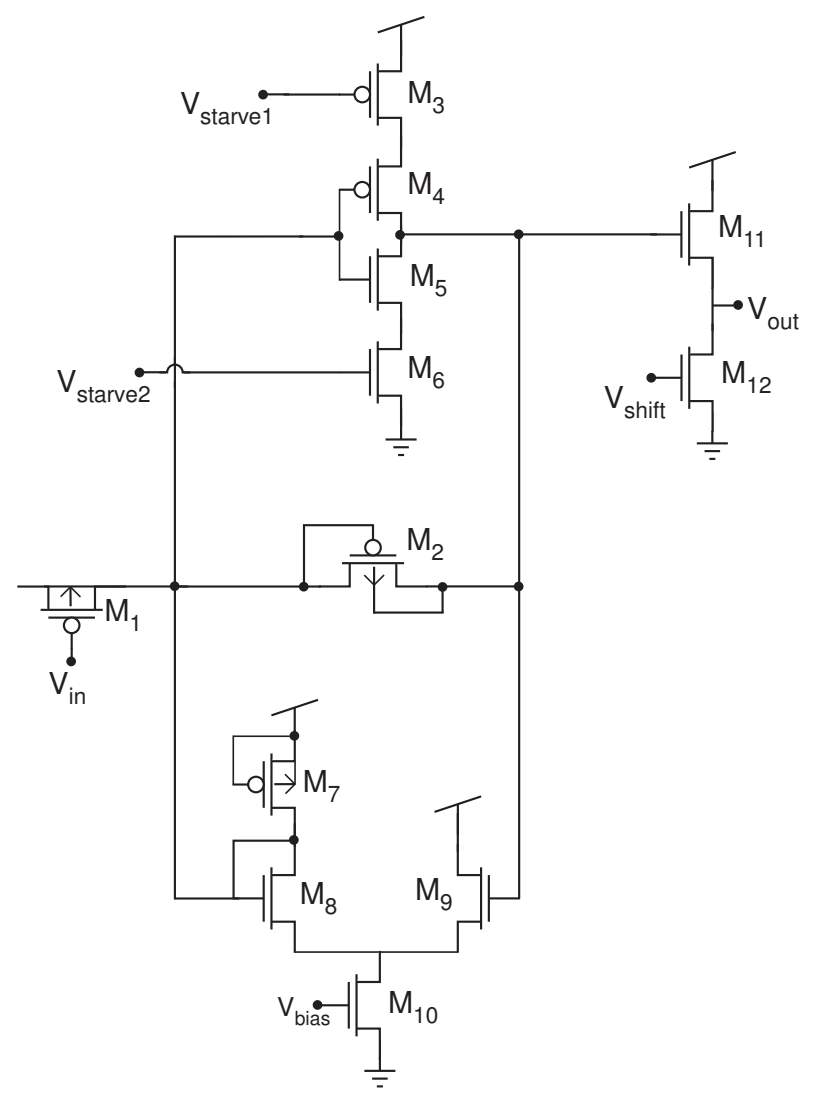

Figure 2.18: The differentiator circuit with current starving circuit and output level shifter

pMOS transistor with the source, drain and gate tied together has been used as the capacitor element in place of a poly-poly or a metal-insulator-metal (MIM) capacitor. By using the MOSFET as capacitor, the real estate required for the circuit is reduced and makes it very compact. The switch of the capacitor from traditional two layer design to the pMOS based design has not affected the performance of the circuit. The amount of capacitance can be controlled by altering the total gate area of the MOSFET.

The limiting circuit used to keep the active resistor from reaching saturation, is built using a very simple element with a sinh-like I-V characteristic (Fig. 2.19) called a Tobi Element [11]. It basically can be viewed as diode-connected well transistor from one direction and parasitic bipolar transistor from the other. When the well voltage is higher than the gate voltage, it behaves like a diode-connected MOS transistor. On the other hand, when the gate is at a higher potential than the well, the p-n junction is forward biased and it acts like a 


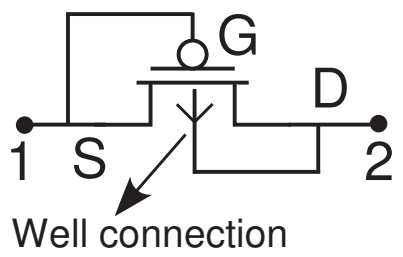

Figure 2.19: The Tobi element

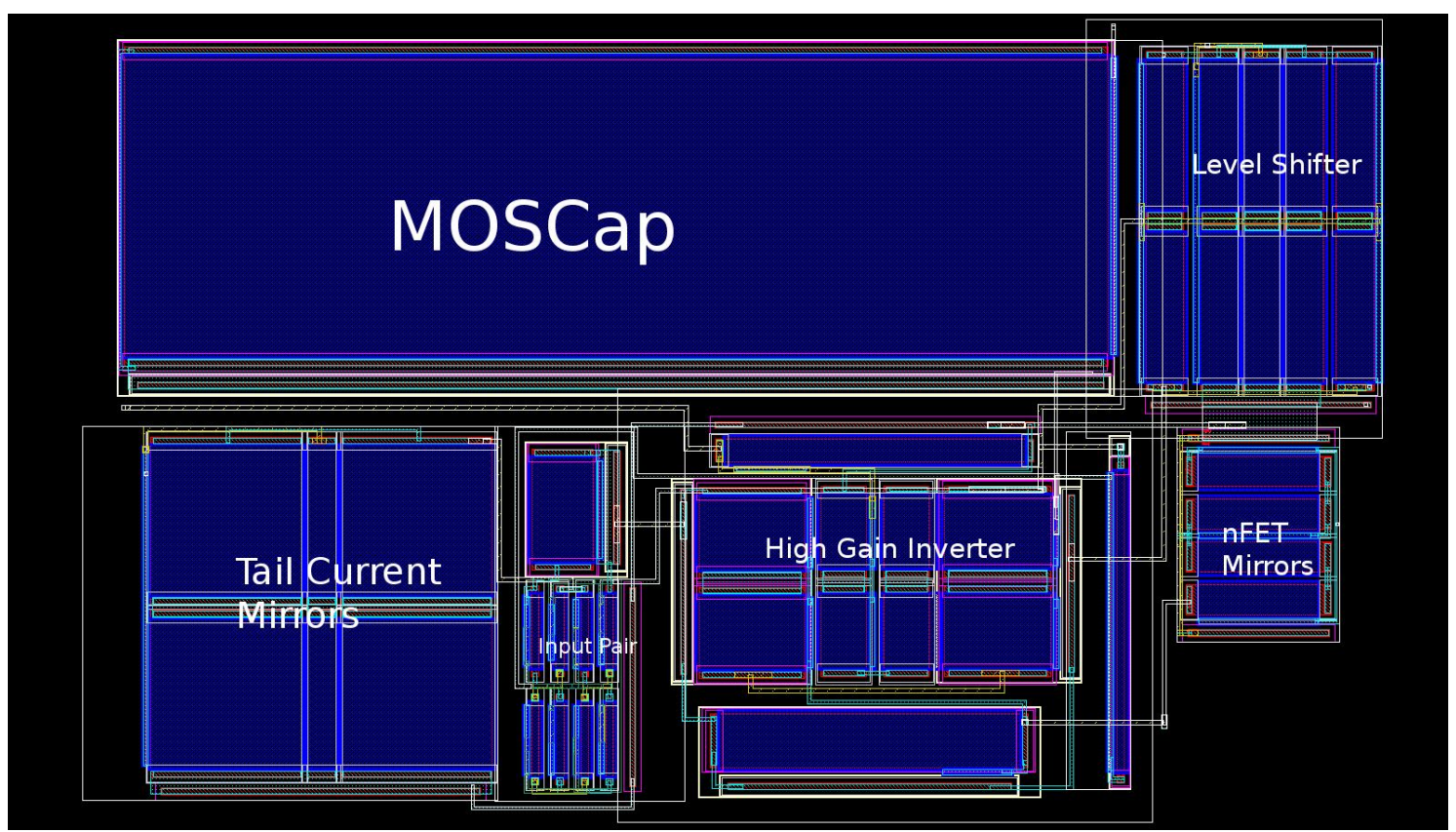

Figure 2.20: Layout of differentiator block

bipolar transistor. The current increases exponentially with voltage on either side of origin, but at the origin, the resistance is very high resulting in a very compact and efficient way to produce a highly resistive element. If an even higher resistance is required, these elements can be arranged in series to increase the resistance additively. This simple and compact circuit is used to limit the charge on the active resistor and thereby limiting it from going into the zone where it loses its resistor like functionality and the current flowing through it becomes constant.

The high-gain inverting amplifier is biased using an active resistor. The high gain is required to bump the output such that the gain in the pass band is unity and also, the 
"differentiation phase" of $90^{\circ}$ is achieved. Also, the current to the inverter is limited using a current-starving configuration as can be seen in (Fig. 2.18). This is done to have control on the amount of current to the inverter, thereby providing control over the total power consumption of the inverter. In general, the inverter is a comparatively high-power-consumption circuit. In this way, the circuit could be tuned to function in low-power consumption mode and at normal performance. This performance trade-off is due to the lower gain on the output resulting in the total shift of frequency response, resulting in a lower gain than unity for the output. This in turn results in loss of the perfect $90^{\circ}$ phase difference between the input and output. In a few applications which require only the temporal change patterns, such small loss can be tolerated for lowering power consumption. And for situations where the phase and gain have to be precise, a high power mode can be used. By tuning the circuit such that the inverter can operate freely without limiting the current through it, the total power consumption increases but the desired performance can be achieved. The active resistor is designed based on a diff-pair. This active resistor sets the operating bias conditions for the inverting amplifier. The bias on this circuit is used to tune the differentiator for operation in different pass-bands.

For differentiating signals of different frequencies the bias conditions need to be varied. The output steady-state voltage of the derivative circuit varies with bias conditions. This change in the steady-state output voltage is due to varying bias conditions of the inverting amplifier, and thus, resulting in a different DC level of the output for each frequency range. To tie the output DC level at a constant level, an extra level-shifter circuit is added at the output stage (Fig. 2.18) of the basic differentiator circuit. This circuit has its own bias and can be tuned to shift the steady state value of the output as per the requirements of a particular application. The layout of the differentiator block is shown in Figure 2.20 and it measures $51 \mu m$ by $84 \mu m$.

\subsubsection{Results from the Differentiator Circuit}

Figure 2.21 shows the basic operation of the differentiator circuit for a sinusoidal input: a sinusoidal input is shifted in phase by $90^{\circ}$ to get its differentiated output in time do- 


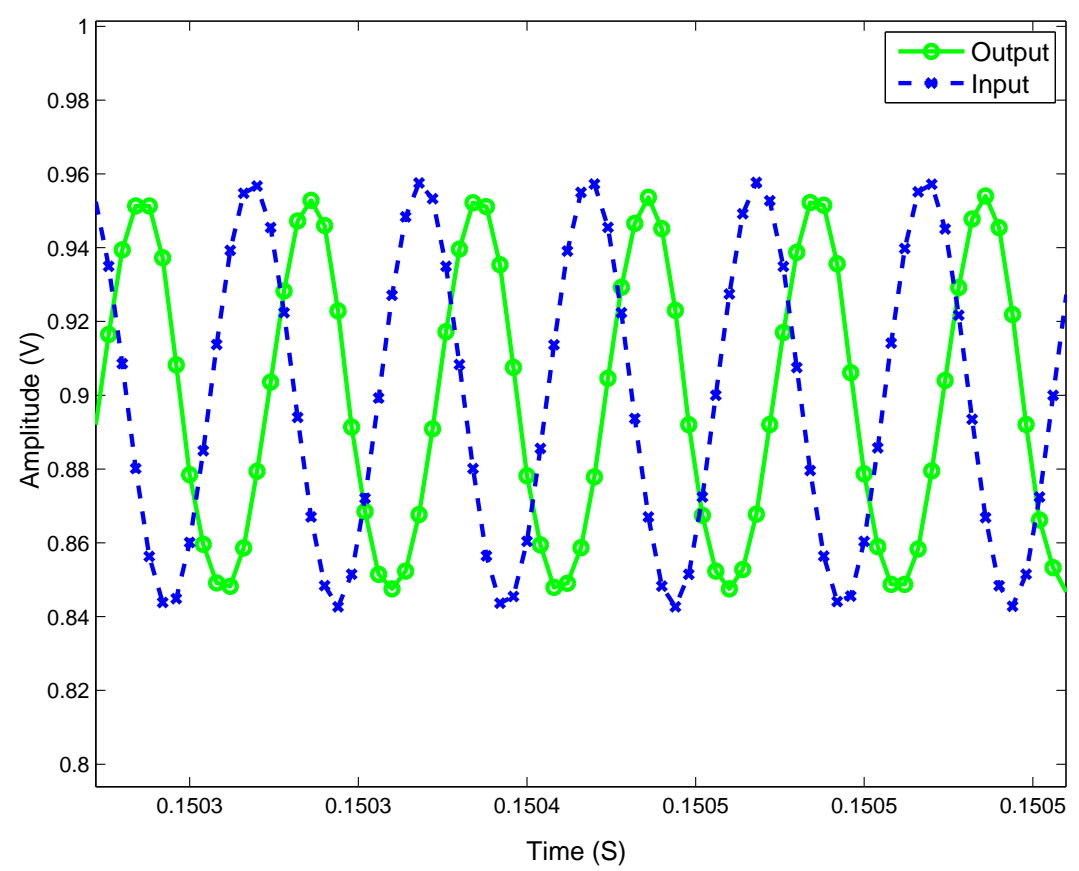

Figure 2.21: Demonstration of sine to cosine conversion by the differentiator circuit

main. Figure 2.22 shows the differentiator frequency response at $20,200,2 k, 5 k$ and $20 k H z$ frequency taps. It can be observed from Figure 2.22 that the gain is set to unity at the corresponding frequencies so that the phase shift remains $90^{\circ}$ for all the frequencies below the corner frequency. Figure 2.23 shows the signal-to-noise ratio of this circuit at different frequency taps. The noise in this circuit is comparatively higher, due to the high gain required to bump up the signals to have a unity gain at the output. It can be observed from Figure 2.23 that the noise is lower as the frequency increases. This is due to the fact that the gain is very low for most of the testing frequency range, resulting in lower noise levels. But in view of the applications of this particular circuit, the noise levels are acceptable as the circuit is used mainly to find any temporal changes in only band-passed signals and it should not affect the overall performance of the system.

Figure 2.24 shows the total harmonic distortion (THD) for the output of differentiator circuit, as a function of the input amplitude. Total harmonic distortion (THD) of a signal is the ratio of the sum of the powers of the second and higher harmonic components of a 


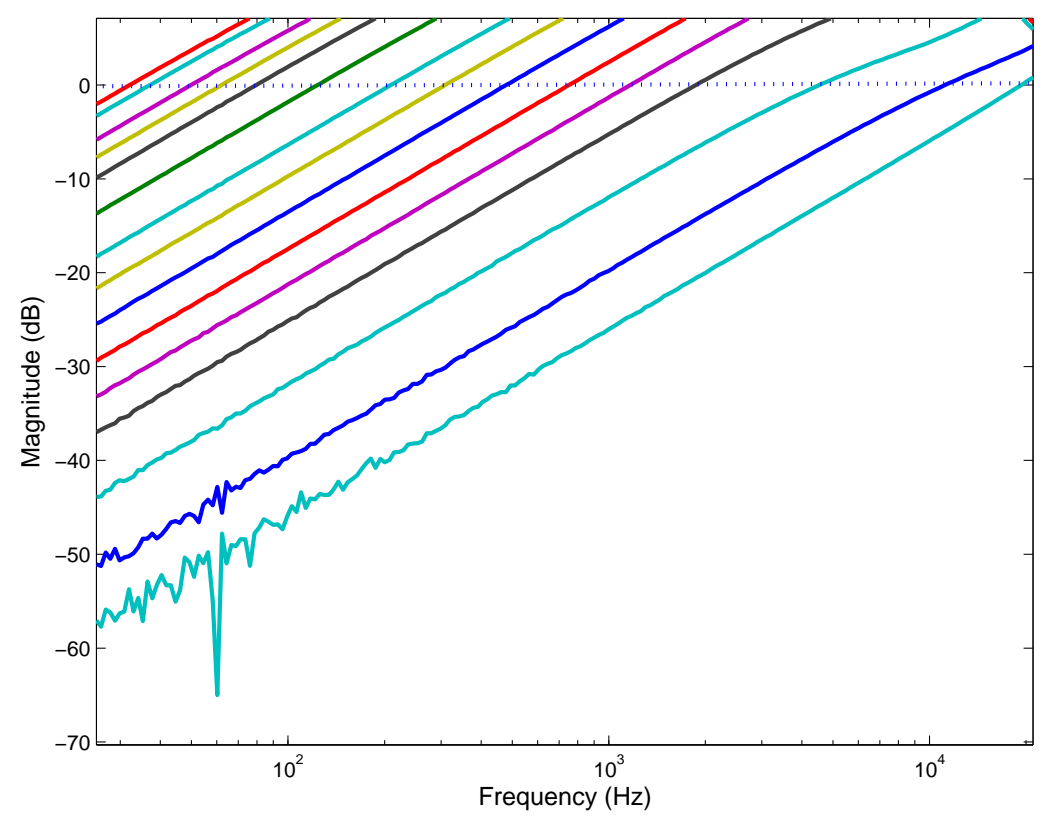

Figure 2.22: Frequency response of differentiator circuit

signal to the power of the fundamental for that signal. It can be expressed as,

$$
T H D=10 \log \frac{V_{h 2}^{2}+V_{h 3}^{2}+V_{h 4}^{2}+\ldots}{V_{f}^{2}} d B,
$$

where $V_{f}$ is the fundamental of the signal and $V_{h 2}, V_{h 3}, V_{h 4}$, etc. are the higher harmonic components. In terms of percentage,

$$
T H D=\frac{\sqrt{V_{h 2}^{2}+V_{h 3}^{2}+V_{h 4}^{2}+\ldots}}{V_{f}^{2}} \times 100
$$

Now, in case of the differentiator circuit, the harmonics of a particular signal, would always have a higher gain, compared to the fundamental. For instance, the differentiator circuit tuned to operate at $1 \mathrm{kHz}$ has a unity gain at that frequency, but the harmonics, at $2 \mathrm{kHz}$, $3 k H z, 4 k H z, 5 k H z, \ldots$ This circuit would have a very high gain, increasing exponentially. This results in the harmonics having higher power than normally they would, thereby resulting in higher THD. Also, this circuit is mainly used to only extract the information about temporal changes at various frequencies of an input signal and apply this knowledge in extracting other useful information. Therefore, this circuit is a very good solution for the application required in this project as it gives a very good approximation of the derivative of an analog input. 


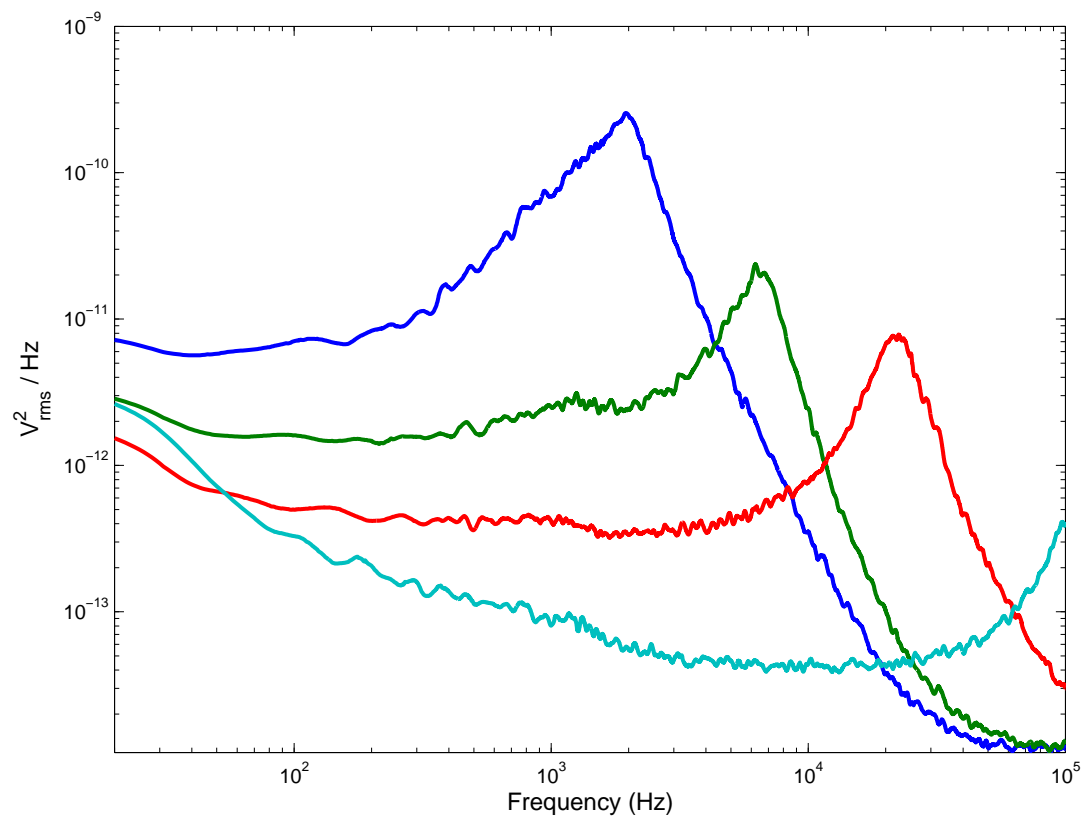

Figure 2.23: Noise power in the output of differentiator circuit

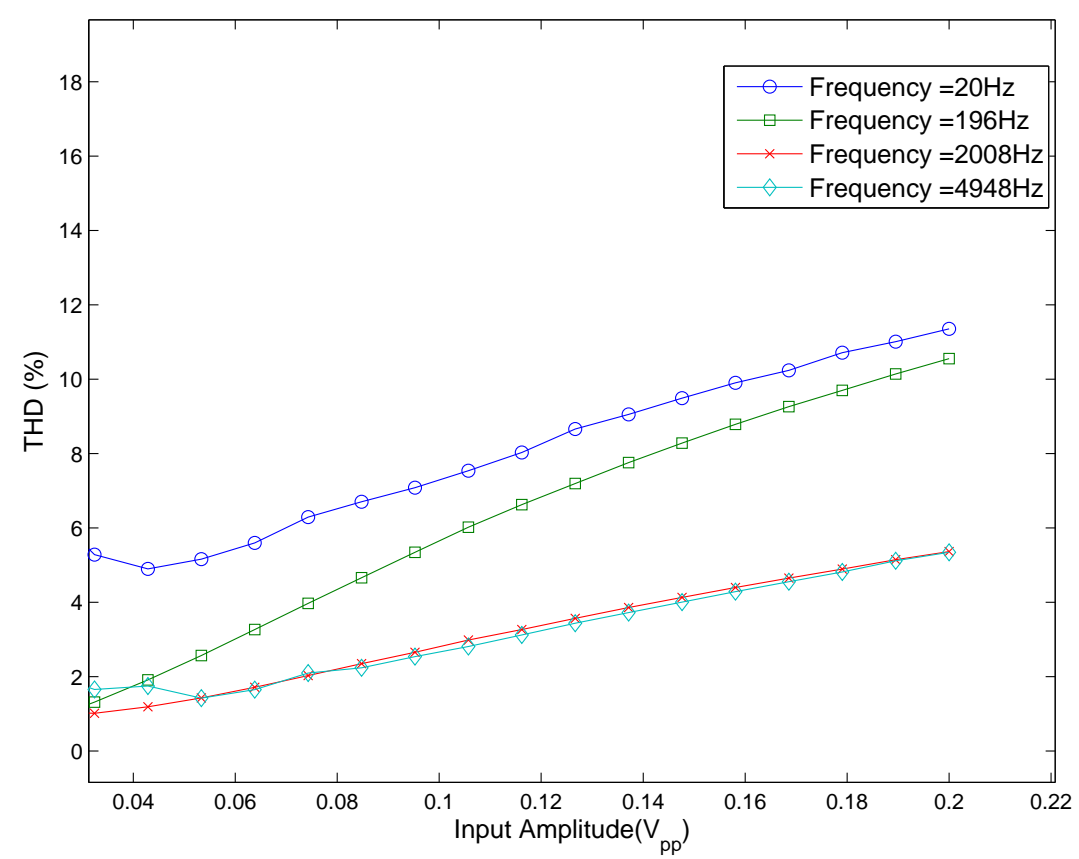

Figure 2.24: THD of the differentiator circuit 


\section{Chapter 3}

\section{Subtractor Circuit}

In general, voltage-mode adders and subtractors form a very essential part of collective analog computational systems [12]. To perform a few application specific tasks on the subbanded signals, a voltage-mode subtractor circuit is required. In this project, analog signals are first spectrally decomposed, and then various signal processing algorithms are applied on them to extract the required information. These processed signals are then sent to the analog-to-digital converter and then to the digital signal processor for further processing. The first phase of processing is done using the analog front-end designed in this project. As part of this front-end, a voltage-mode subtractor is designed.

The subtractor circuit designed here is for low-power consumption and high-dynamic range. The power consumption is in $\mu W s$ and the dynamic range is $64 d B$. Also, the circuit is very easily tuned as it works over the whole audio range using only one bias value; that is, it does not need a seperate bias for each single tap in the larger system. This chapter describes the design and operation of the subtractor circuit.

\subsection{Basic Principle}

As current can be easily added or subtracted based on Kirchoff's current law, a voltagemode subtractor circuit could be designed by first converting voltage signals into current and then converting them back to voltage after the required operation has been done. This conversion can be done using an operational transconductance amplifier (OTA) or current 


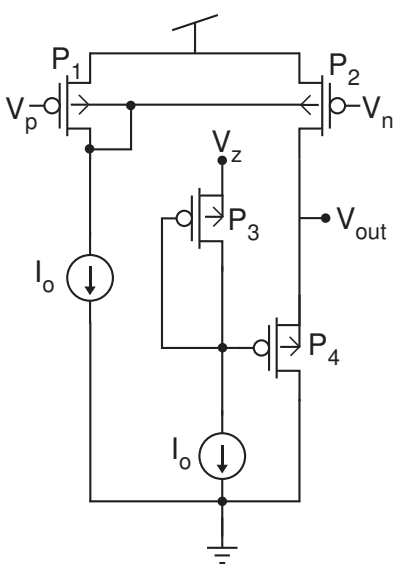

Figure 3.1: Basic idea for voltage-mode subtractor circuit

conveyors [13]. The circuit used here was first reported by R. Fried and C. Enz in Electronics Letters, 1997 [4]. An alternative approach is presented in [14]. In this alternate method, there are limitations on the input voltage levels to prevent the transistors in the circuit from getting cut off or getting saturated. But the main limiting factor is that, the ciruit presented in [14] operates in strong-inversion, making it unsuitable for low-power applications. Only a single MOSFET is used to convert the voltage to current for each input and even the output current is transformed to voltage using only a single such MOSFET. The basic requirement here is to make use of all four terminals of a MOSFET. The p-FETs give us the freedom to access and alter the voltage of its backgate, or the well terminal, without varying any other voltages of the system. Therefore, this circuit is p-FET based.

\subsection{Design of Subtractor Circuit}

Figure 3.1 shows the basic working priciple of the subtractor. In this circuit, all of the transistors are biased in weak inversion (also in saturation) and have the same gate areas. For a p-FET in weak inversion and saturation, the drain current is given by [15],

$$
I_{D}=I_{d O} e^{\left(\frac{\kappa V_{g}-V_{s}}{U_{T}}\right)}
$$

where $I_{d O}$ is the leakage current, $U_{T}=k T / q$ and $\kappa$ is the slope factor. Since all the tranistors have the same gate area (same aspect ratio), $I_{d O}$ can be safely assumed to be the same for 
all the transistors. This gives the relation between the bias current and output as,

$$
\frac{I_{\text {out }}}{I_{\text {bias }}}=e^{\left(\frac{\kappa\left(V_{p}-V_{n}\right)}{U_{T}}\right)}
$$

From equations 3.1 and 3.2, the voltage at the output transistor, $P_{4}$ (Fig. 3.1) is given by,

$$
V_{\text {out }}=V_{p}-V_{n}+\left(\frac{\kappa}{U_{T}}\right) \ln \left(\frac{I_{\text {bias }}}{I_{d O}}\right)+V_{g},
$$

where $V_{p}$ and $V_{n}$ are the input voltages and $V_{g}$ is the gate voltage of $P_{3}$ (Fig. 3.1). The equation 3.3 clearly shows that the output voltage is the difference of the two input voltages along with a small offset voltage $V_{\text {off-set }}$, which is given by,

$$
V_{o f f-s e t}=\left(\frac{\kappa}{U_{T}}\right) \ln \left(\frac{I_{b i a s}}{I_{d O}}\right)+V_{g}
$$

The gate voltage of the transistor $P_{3}$ (Fig. 3.1) is given by,

$$
V_{g}=V_{z}-\left(\frac{\kappa}{U_{T}}\right) \ln \left(\frac{I_{\text {bias }}}{I_{d O}}\right) \text {. }
$$

Now, from equations 3.3, 3.4 and 3.5,

$$
V_{\text {out }}=V_{p}-V_{n}+V_{z}
$$

This gives us the required subtractor operation. The extra offset voltage, $V_{z}$ can be used to set a constant offset voltage for the output or can even be used as additional input voltage. Therefore, this circuit has the potential to be used as either a subtractor or as an adder.

\subsubsection{Working of Subtractor Circuit}

The main requirement for the application of Equation 3.1 is that the transistors $M_{1}$ and $M_{2}$ remain in saturation. To ensure this, an extra transistor, $M_{6}$, in source follower configuration is used as shown in Figure 3.2. The bulk of $M_{6}$ must be tied to a high voltage $\left(V_{d d}\right)$ which results in the bulks of $M_{1}$ and $M_{2}$ rising higher than $V_{d d}$, increasing the operating range for the input voltage. As the current flowing through $M_{1}$ is constant, the changes in its bulk voltage are twice that of the changes in the gate voltage as can be observed from equation 3.1. Also, the source-bulk junction has to be at a voltage less than the total swing of any of the input voltages (in this case $\approx 400 \mathrm{mV}$ ). Therefore, the bulk voltage $V_{\text {bulk }}$ 


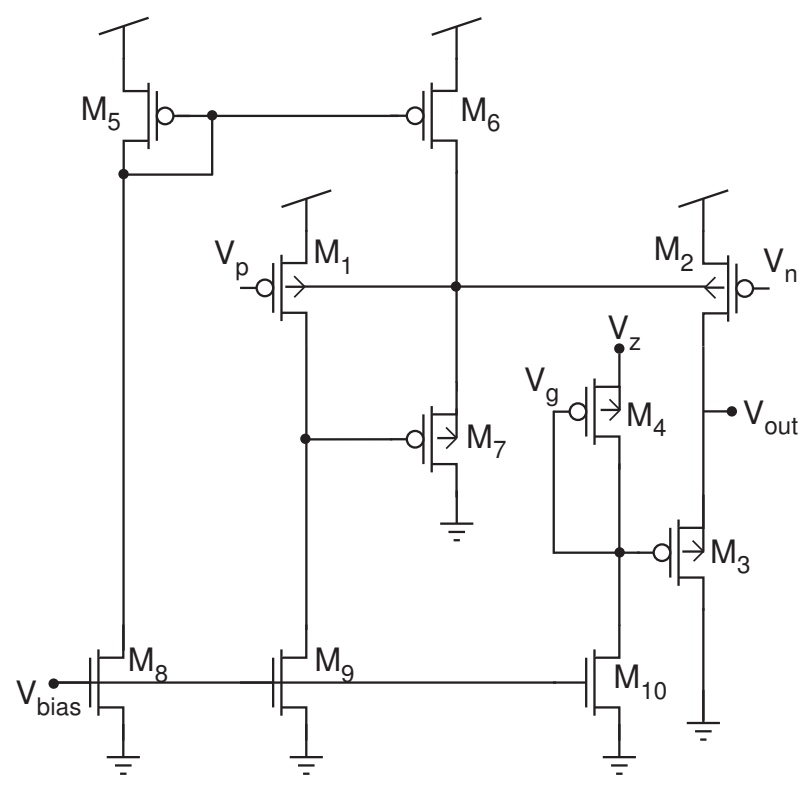

Figure 3.2: Subtractor circuit

needs to be greater than the sum of $V_{\text {Threshold, }}$, the maximum input swing and $V_{\text {Dsat }}$ (that is, $V_{\text {bulk }} \geq V_{\text {Threshold }}+V_{\text {Dsat }}+400 \mathrm{mV}$ ) . For this reason, the bulk can be tied safely to $V_{d d}$. This also eliminates the need of an extra biasing input.

For the subtractor to work with input signal amplitudes ranging roughly from 10 to $200 \mathrm{mV}$ with a $\mathrm{DC}$ value of $0.9 \mathrm{~V}$, the inputs must be level-shifted to a higher DC level than the $0.9 \mathrm{~V}$ input $\mathrm{DC}$ level for this project. This is required as the transistors need to be in saturation. The DC level at which this subtraction occurs can be controlled by increasing the bias current, but only to a certain extent. The level-shifting is done using just two transistors for each input terminal. Initially, it was assumed that all the operational transistors are identical and have the same aspect ratio. But, there can be mismatch created during fabrication and it may result in the transistors having a different saturation current. Therefore, using different $I_{d O}$ for the operational transistors and using equations 3.1 and 3.6, we have,

$$
V_{\text {out }}=\left(V_{p}-V_{n}\right)+V_{z}+\left(\frac{\kappa}{U_{T}}\right) \ln \left(\frac{I_{d O 2} I_{d O 4}}{I_{d O 1} I_{d O 3}}\right)
$$

From 3.7 it can be observed that, the mismatches only add a constant off-set to the output. This can be easily eliminated by using a suitable voltage for $V_{z}$, for compensating the offset. 
Moreover, this circuit requires only one bias voltage for its operation. With a single bias value, it can perform the subtraction over the whole audio range without any requirement of tuning for different frequencies. Also, as the operational transistors are identical, any changes in circuit conditions due to temperature are automatically offset due to the symmetrical nature of the circuit. The noise is level is lower in this circuit as most of it gets cancelled out during the subtraction.

The layout of the subtractor block is shown in Figure 3.4 and it measures $115 \mu \mathrm{m}$ by $147 \mu \mathrm{m}$. As can be observed from Figure 3.4, the core subtractor circuit is very small and the maximum area is used up by the level shifters.

\subsubsection{Output Limitations}

The slope factor of the transistors changes with the change in gate-to-bulk voltage. The upper and lower limit of the differential input voltage is therefore limited. The uppper limit depends on the lower voltage of the bulks of $M_{1}$ and $M_{2}$ at which the source-bulk junction is forward biased. The lower limit depends on the $M_{1}$ tansistor's gate voltage, beyond which the transistor comes out of the weak inversion region. Even with these limits, the required operational range is not much affected, as most of the singals are within $50-200 \mathrm{mV}$. Also, its operating frequency is limited due to the use of MOSFETs in weak inversion. However, it works reliably in the region below the upper end of the audio frequency range.

\subsection{Results}

The Figure 3.3 shows the complete implementation of the subtractor circuit. To test the basic operation of the circuit, a sine wave with an amplitude of $100 \mathrm{mV}$ and $1 \mathrm{kHz}$ frequency is given as the input to the positive terminal. To the negative terminal, a similar input with a phase shift of $180^{\circ}$ (relative to the input at positive terminal) is given. Figure 3.5 illustrates the output of the circuit in this scenario. As can be observed in Figure 3.5, the input DC level is shifted to $1.5 \mathrm{~V}$ (from the $0.9 \mathrm{~V}$ input DC level for this project) for the saturation of the operational transistors and thereby facilitating subtraction. The output DC level is set by the voltage $V_{z}$, which acts like an additional input to the circuit. This can 


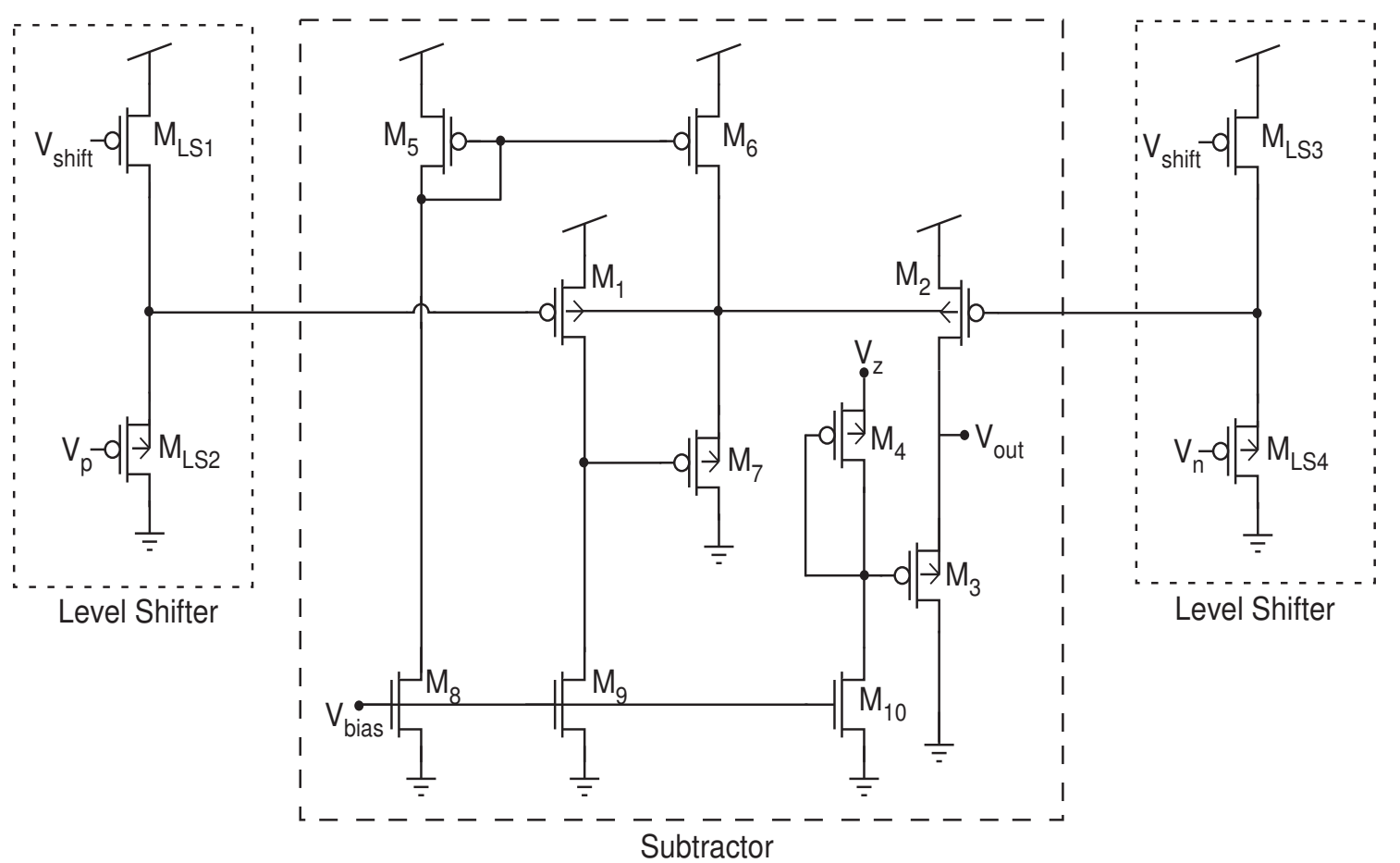

Figure 3.3: Subtractor circuit with level shifted inputs. The two level shifters move the DC level of the inputs to a higher leve to enable subtraction

be used to correct any off-sets in the output DC level. As another illustration of subtraction, an amplitude modulated sine wave is subtracted from a sine wave with $1 k H z$ frequency, and Figure 3.6 illustrates the response of the circuit for such input.

As discussed earlier in Section 3.2.2, the output has limitations in its amplitude range. Figure 3.7 illustrates the amplitude versus error relation for the subtractor for sinusoidal inputs. The error was calculated by comparing the ideally expected output with the real output. The percentage of error also depends on the input and output DC levels of the signal. The higher the DC offset for the input, the lower is the error (Fig. 3.8). The optimum level for this is at $1.5 \mathrm{~V}$, when operating with a supply voltage of $1.8 \mathrm{~V}$. On the output DC level, there is not as much dependence but the performance is slightly better for higher DC levels (Fig. 3.9). With the optimum bias and DC levels, the normalized error is less than $2 \%$ for amplitudes upto $200 m V$ (peak-peak).

The frequency response of the subtractor circuit is illustrated in Figure 3.10. The gain starts to drop below unity for higher frequencies possibly due to the transistors coming out 


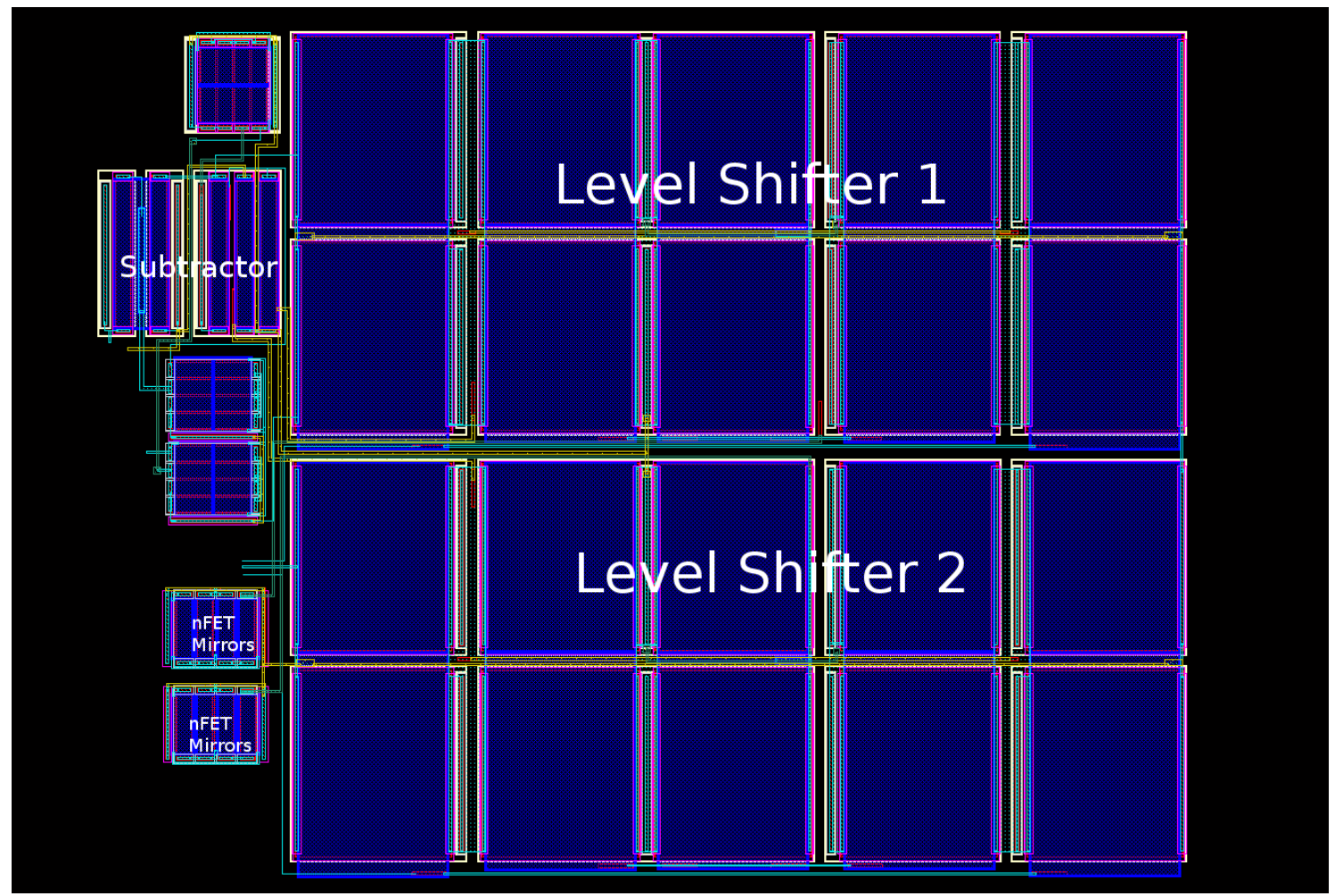

Figure 3.4: Layout of subtractor block

of the weak inversion region. Also, the buffer used at the output of the subtractor block (for testing of this circuit) has limited performance. These reasons limit the performance at higher frequencies but the circuit works perfectly for all other lower frequencies.

The total harmonic distortion (THD) of the circuit is also well below $1 \%$ for even the amplitudes of up to $200 \mathrm{mV}$ (peak-peak). The THD also varies with the frequency of operation. As the frequency response starts to go into non-ideal zone for higher frequencies, THD also suffers for the signals at these frequencies. Figure 3.11 shows the THD for the circuit for varying amplitude at various frequencies in the audio range. The THD also depends on the input DC level, as it affects the maximum frequency at which the transistors go out of weak inversion region. The signal-to-noise (SNR) ratio of the circuit varies with the operating frequency. But the variation is within $0.5 d B$, with the mean of $60 d B$. The Figures 3.12 and 3.13 illustrate the SNR at various frequencies and the variation of SNR with the bias voltage of the subtractor. Slight variations in bias voltage do not affect the SNR level appreciably. 


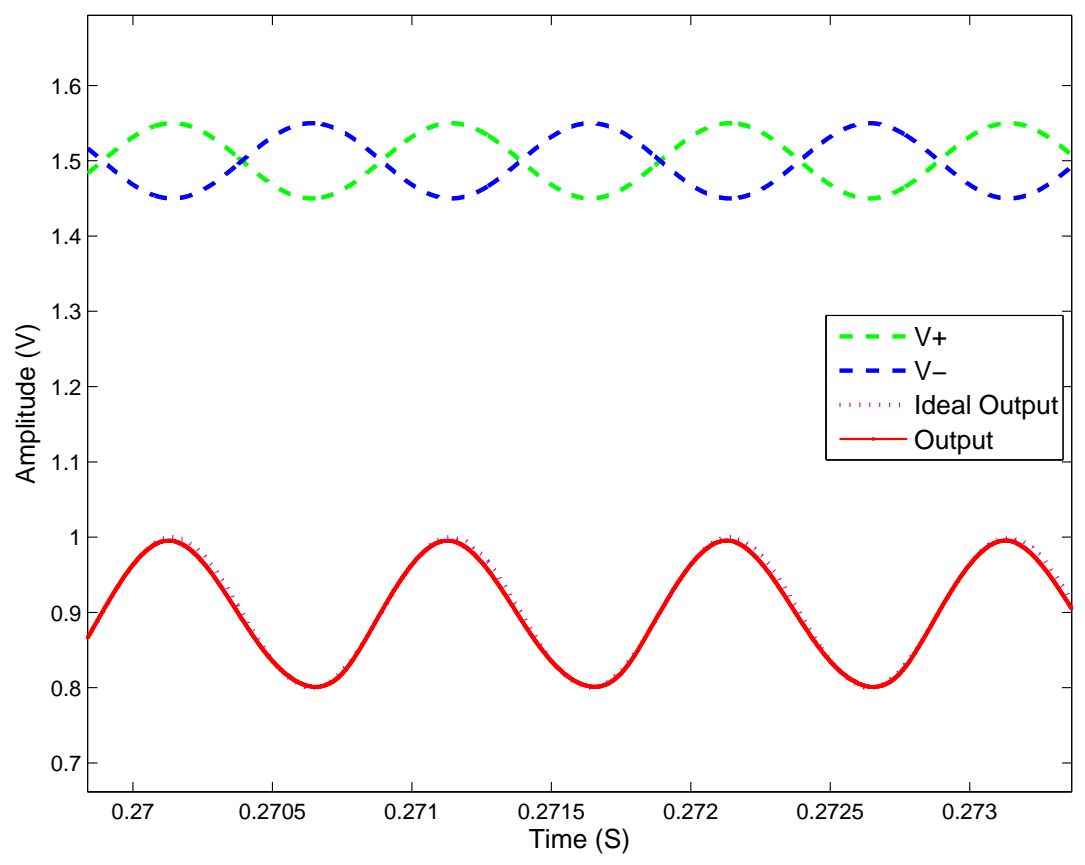

Figure 3.5: Voltage-mode subtraction of two sinusoidal signals

The next figure of merit considered to measure the performance of this circuit is the dynamic range. It has been calculated by measuring the highest peak voltage input for which the THD is less than $1 \%$ and dividing it by the noise floor of the circuit. The total dynamic range in $\mathrm{dB}$ is given by,

$$
\text { DynamicRange }=10 \log \left(\frac{\text { Maximum output }}{\text { Noise floor }}\right)
$$

The dynamic range for this circuit is $63.94 \mathrm{~dB}$. The overall power consumption of the circuit is also within $2 \mu W$ for any frequency tap, as the bias voltage is essentially the same for all frequencies. 


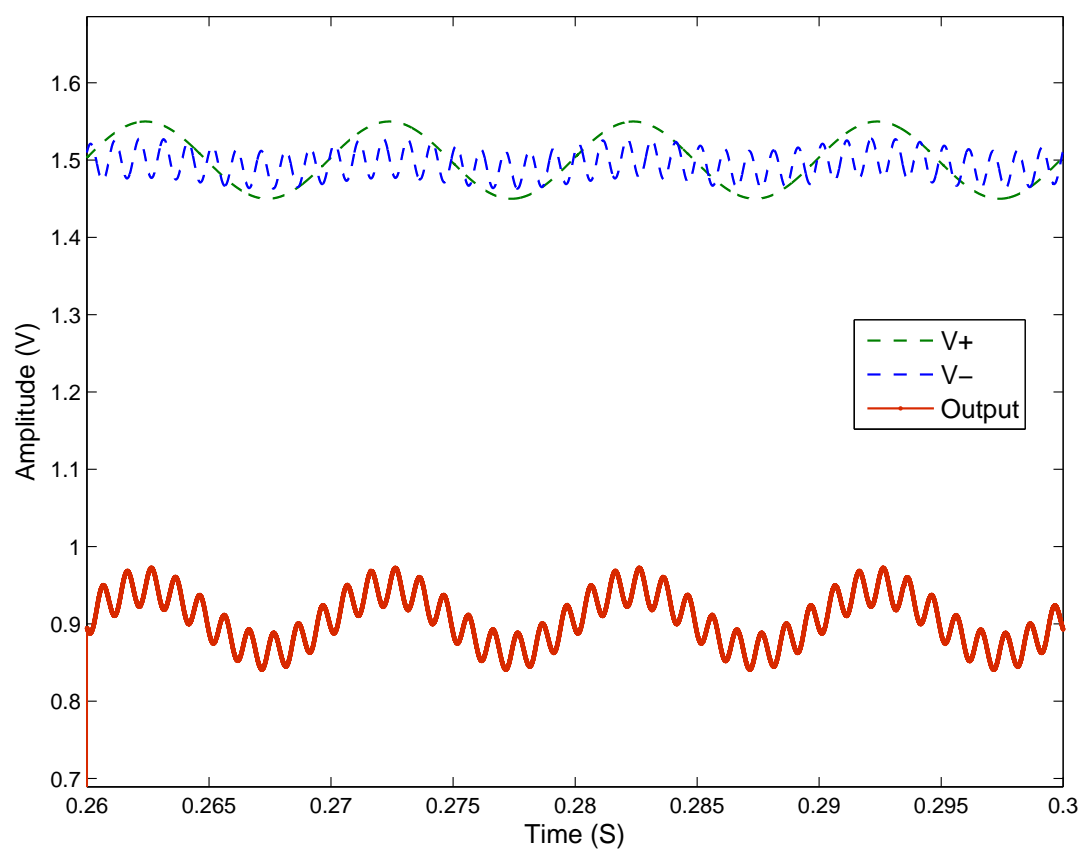

Figure 3.6: Demonstration of Voltage-mode subtraction with amplitude modulated signal as input.

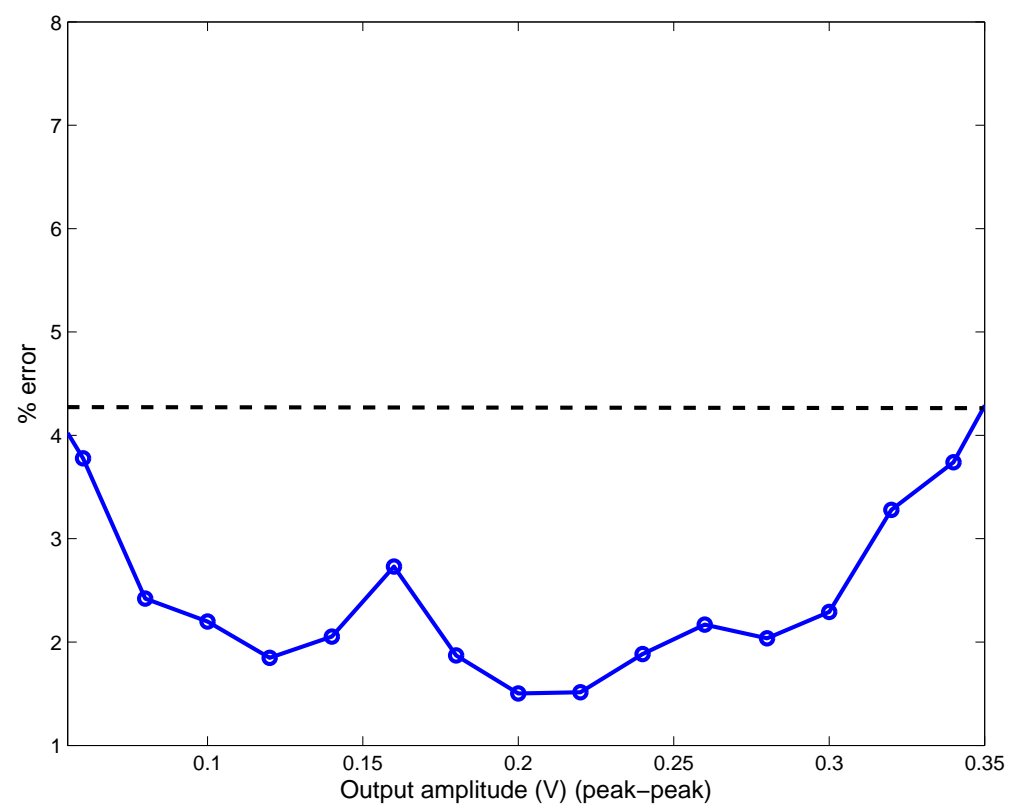

Figure 3.7: Error versus input amplitude for the subtractor circuit 

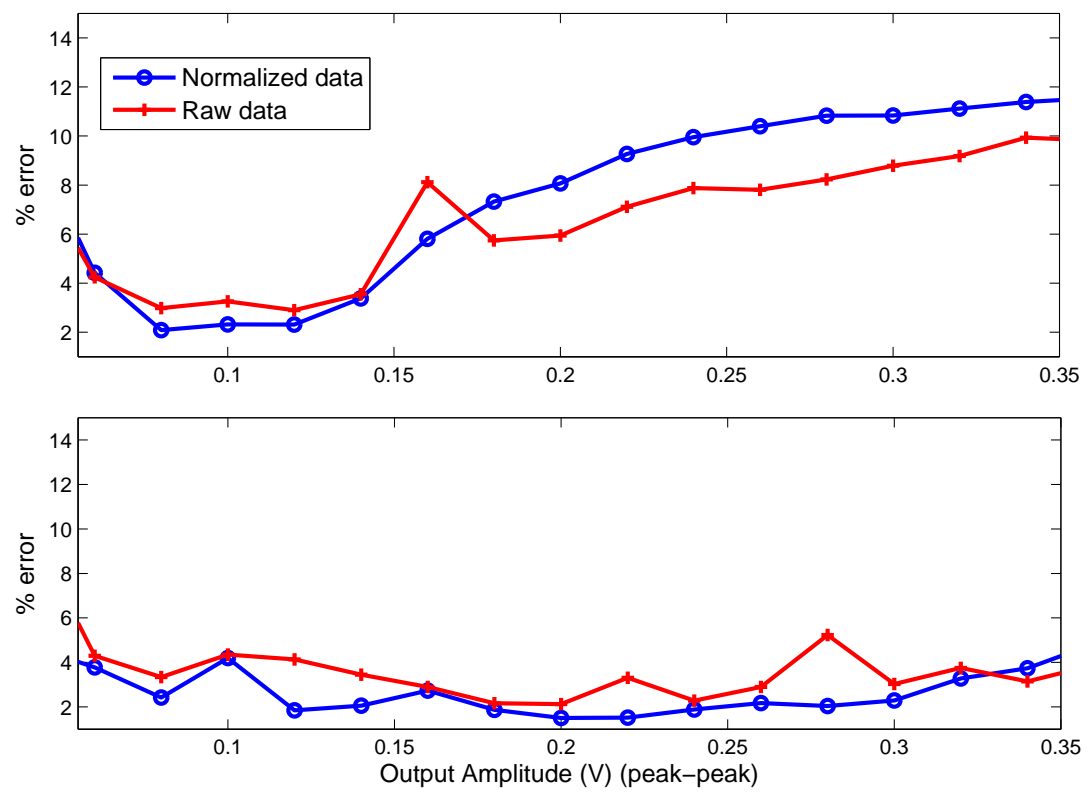

Figure 3.8: Higher input DC level gives lower \% error, (a) has a lower input DC compared to (b).
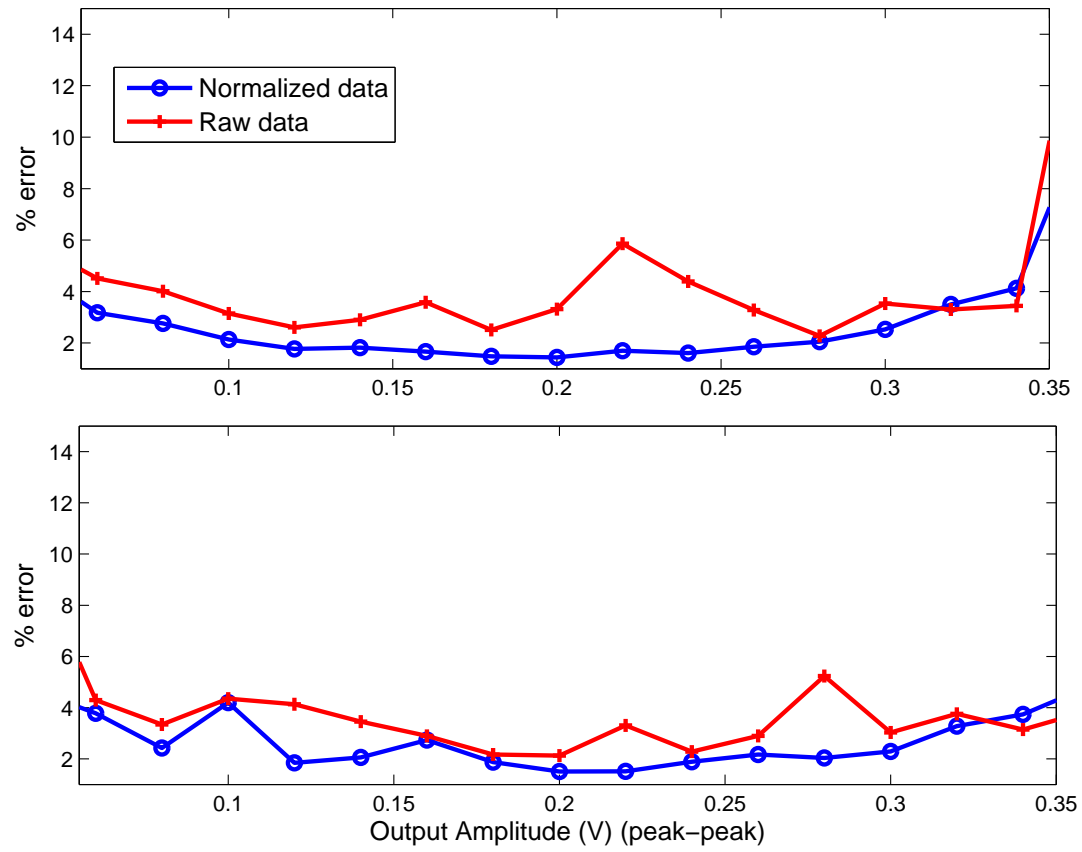

Figure 3.9: Higher output DC level gives slightly lower \% error, (a) has a lower output DC compared to (b). 


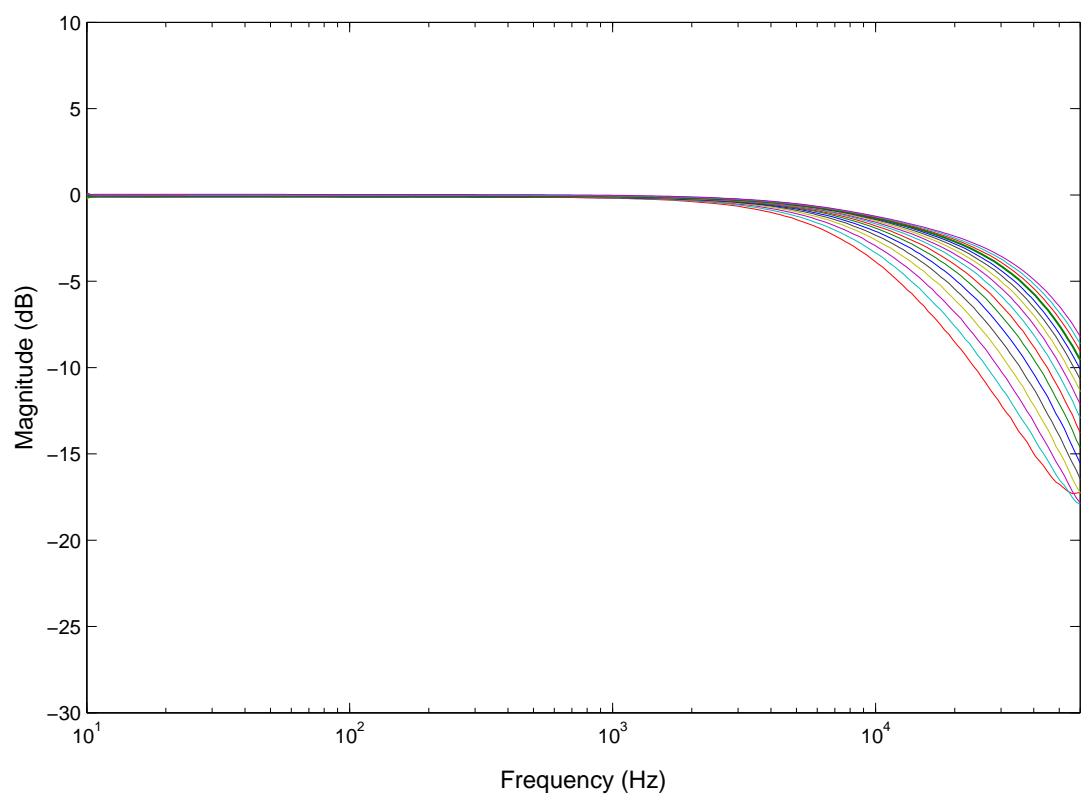

Figure 3.10: Frequency response of subtractor circuit. The gain is unity for all the frequencies in the audio range. It only starts to drop slightly at higher frequencies.

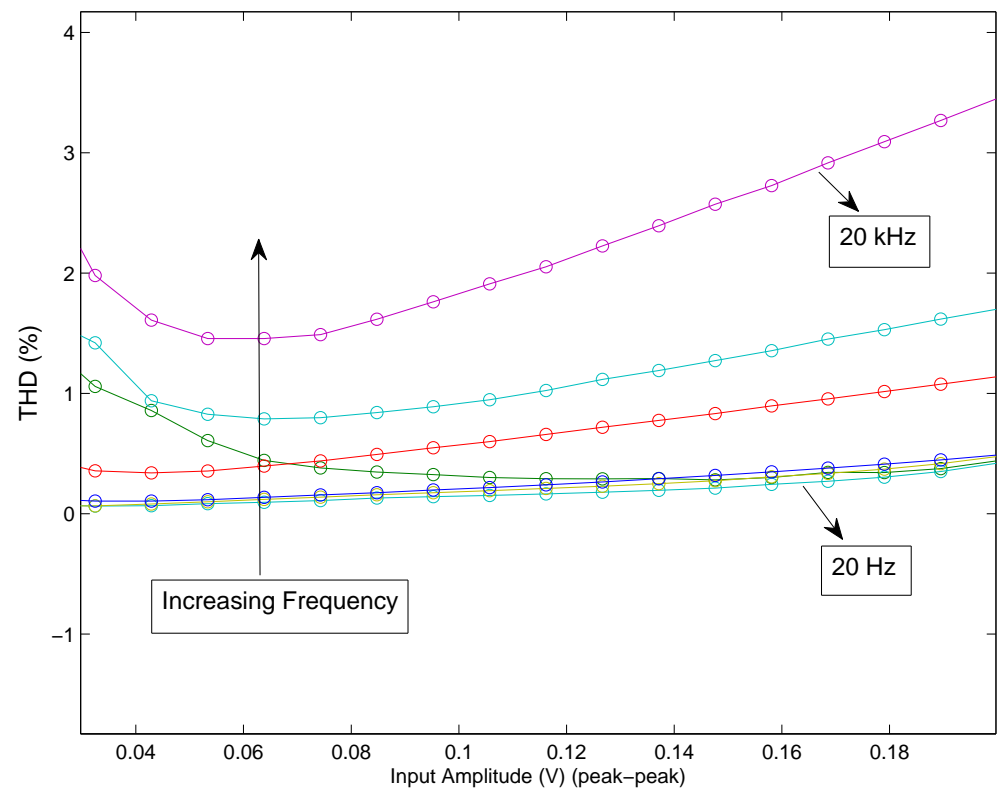

Figure 3.11: THD of subtractor circuit. 


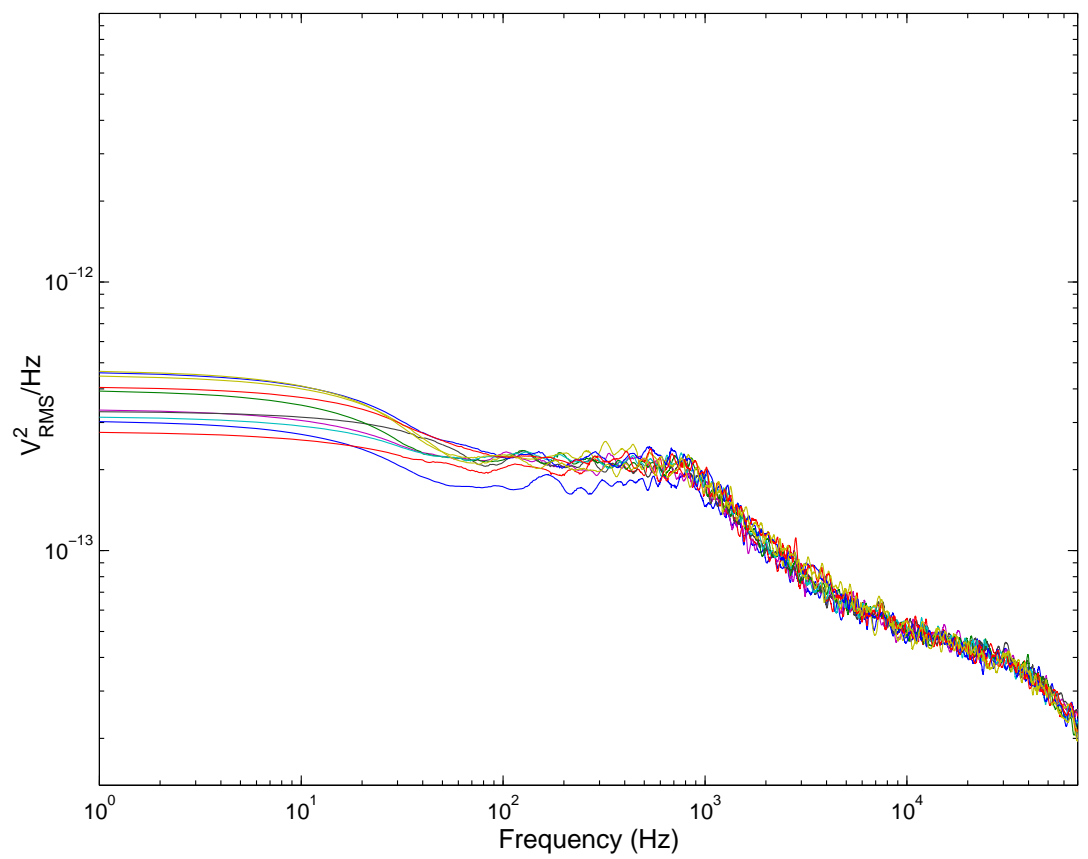

Figure 3.12: Noise power in the output of the subtractor circuit

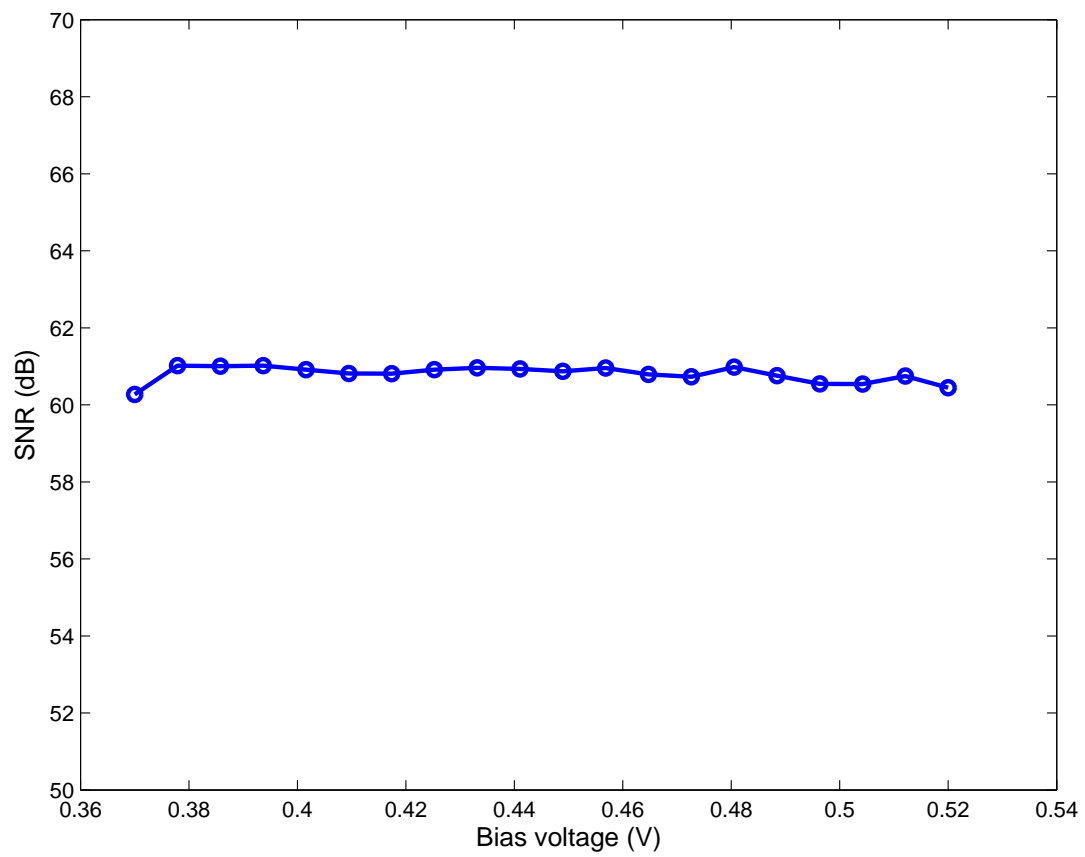

Figure 3.13: Variation of SNR with the bias voltage of the circuit 


\section{Chapter 4}

\section{Floating-Gate Tranistors}

\subsection{Floating-Gate Transistors}

To program the circuits dynamically and to allow post-fabrcation reconfigurability we need non-volatile memory elements which can store the charge to preserve the biasing conditions and the inter-block connection configuration. For this, the floating-gate transistors (Fig. 4.1) are used. A transistor in which the gate is electrically isolated can be defined as a floating-gate transistor. The flow of electrons to and from the gate is restricted due to this isolation. The secondary control-gate (Fig. 4.1) capcitively couples charge onto the isolated (floating) gate and this results in the shift of the MOSFET's tranfer function as illustrated in Figures 4.2 and 4.3

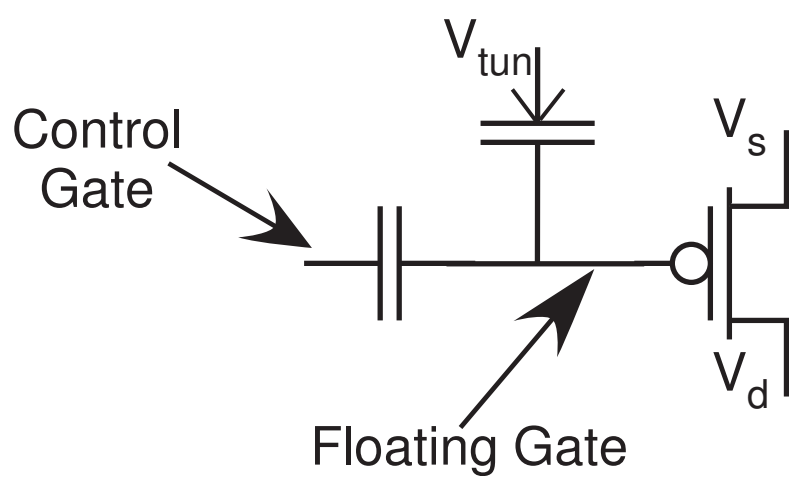

Figure 4.1: Floating gate and Control gate 


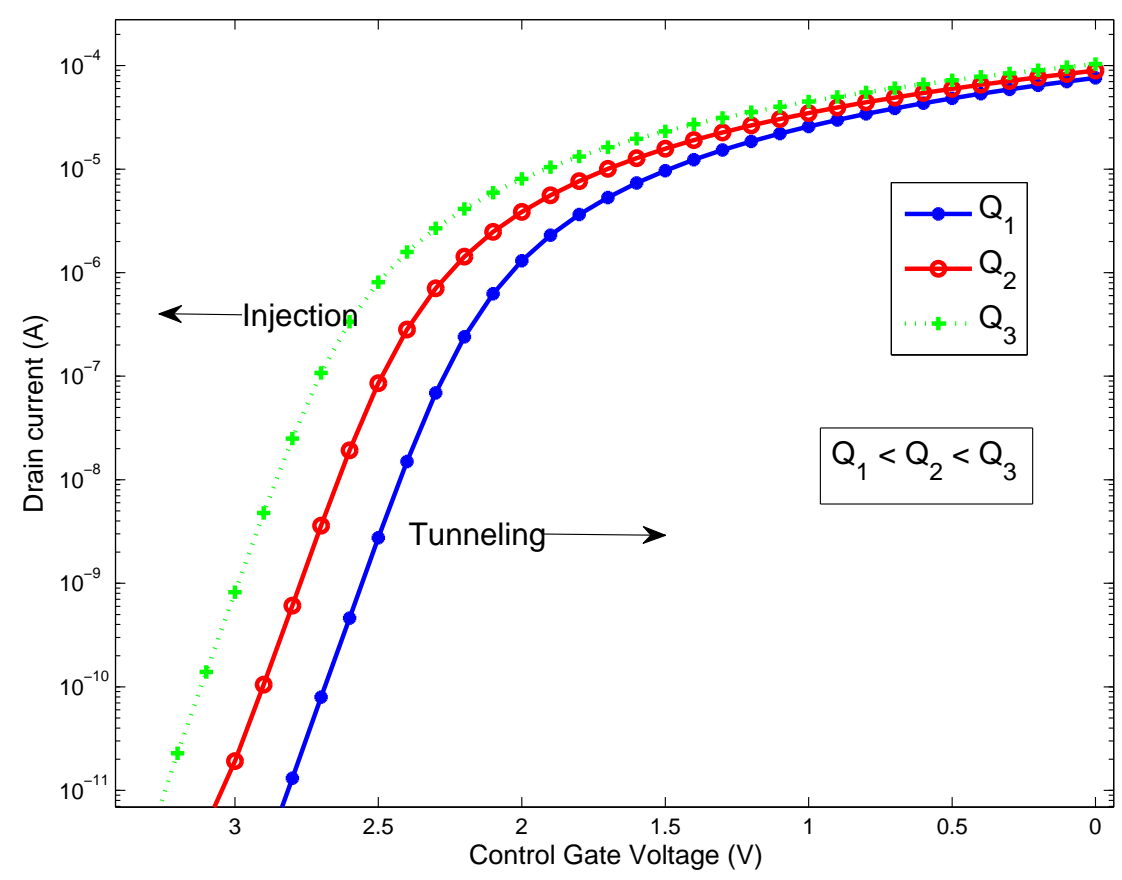

Figure 4.2: I-V relation with charge present on the floating gate

Mostly to modify the floating-gate charge, two basic mechanisms are used: Hot-Electron Injection and Electron Tunneling (A different approch to this is using UV light [16], but this is not feasible for our application). Tunneling is used to completely remove the charge from the gate and make it electrically fresh to be programmed with a new gate voltage. HotElectron Injection is used to add charge onto the gate, thereby controlling the gate voltage which results in regulation of current flowing though the transistor. These two procedures are breifly explained in the following sub-sections.

\subsubsection{Electron Injection}

To move the elctrons over the Silicon Dioxide $\left(\mathrm{SiO}_{2}\right)$ barrier, the electron affinity difference between the gate material (a metal) and the $\mathrm{SiO}_{2}$ has to be overcome. In this process, the electrons are forced to go over the barrier by exciting them or making them hot [6]. This is shown in Figure 4.4. Basically, there are two requirements for the electron injection to take place. First, the elctrons must possess the required potential $(3.1 \mathrm{eV})$ to overcome the 


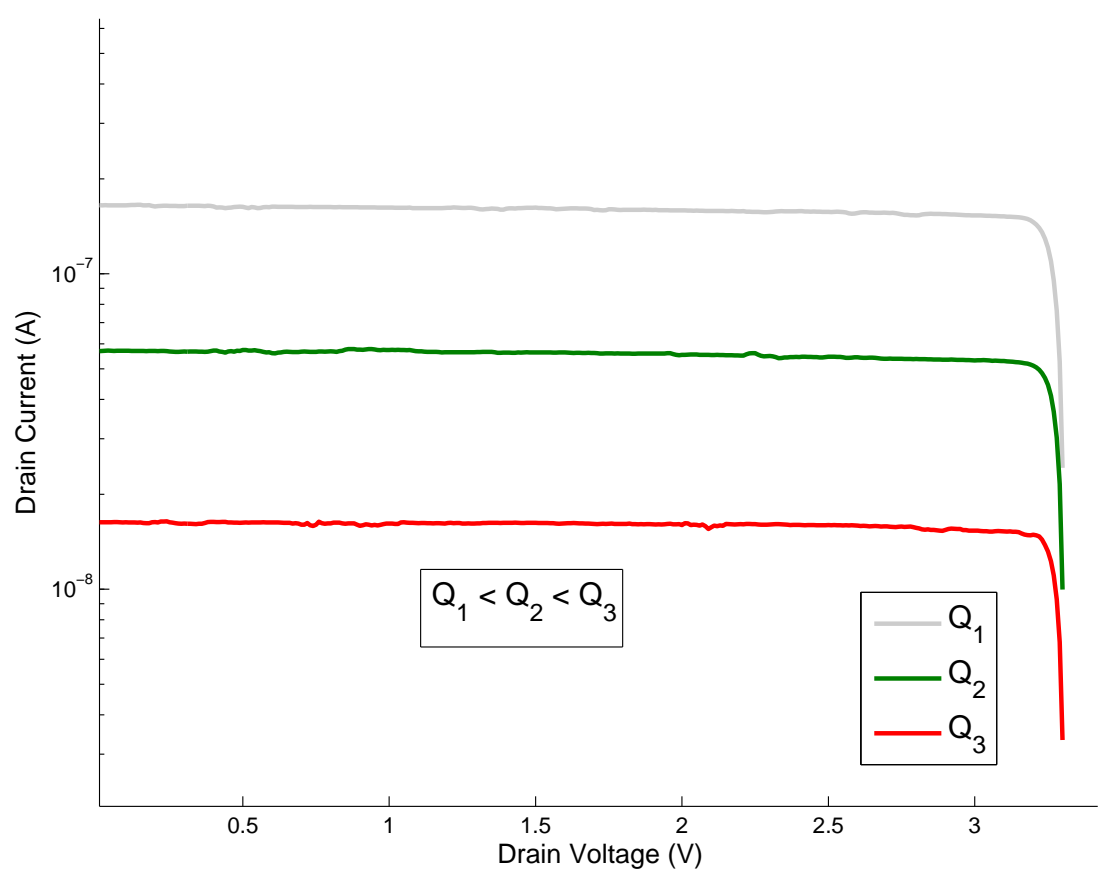

Figure 4.3: The drain current can be varied by changing the floating-gate charge. A constant value can be set and used to set bias different circuits

elctron affinity difference between $\mathrm{SiO}_{2}$ and $\mathrm{Si}$. Second, the oxide electric field should be in the direction required to transport injected electrons to the floating gate.

For injection to happen satisfactorily, there have to be enough hot-electrons created first. In case of conventional nFET based floating-gates, a special bulk p-type implant is required to increase the hot-electron population in the depletion region. Such doping requires more processing and is not possible on monolithic systems. Moreover, in nFETs, to prevent stray electrons from the drain being collected on the gate of the transistor, an extra padding is included on either end of the gate. This extra padding is also known as lighlty doped drain and is useful to collect the stray electrons. Such padding is not present on the pFETs as the electric field direction would pull the electrons onto the gate even in presense of a padding and is useful in electon injection. Therefore, in this project, the pFET based floating-gates are used. In a sub-threshold pFET with high source-to-drain potential, the channel-to-drain voltage is large and this causes holes to collide and create free electronhole pairs. These generated electrons can be collected on the floating gate by satisfying 


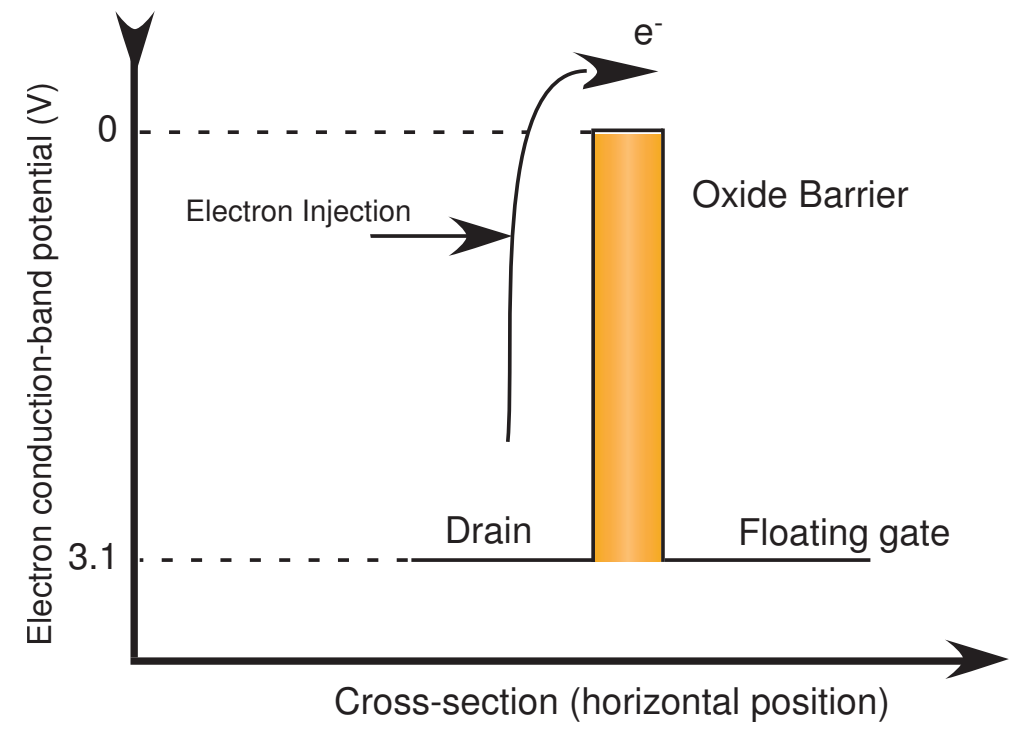

Figure 4.4: Electron injection

the two required conditions mentioned earlier in this section. The first condition is met by using a suitably large source-to-drain voltage. And the second condition is already in place for this configuration as in a subthreshold MOSFET, the source-to-drain potential is a few volts higher than the source-to-gate potential. This leads to the floating-gate being at a higher potential than drain and this electric field naturally transports the electrons to the floating-gate. In this way, the charge on the floating-gate can be controlled by slowly injecting electrons by stepping up the corresponding voltages.

\subsubsection{Electron Tunneling}

The electrons can be made to overcome an $\mathrm{SiO}_{2}$ barrier, by pushing the elctrons through it. This process is called electron tunneling. The particular form of tunneling described here is the Fowler-Nordheim tunneling [7]. In this process, the barrier is the $\mathrm{SiO}_{2}$ gate oxide. On one side of this barrier is the polysilicon gate and on the other side is a heavily doped silicon surface (Fig. 4.5). A high potential difference between these two sides reduces the effective thickness of the silicon barrier and thereby facilitates tunneling. In this way, electrons can be moved from the floating-gate. The electrons can also be moved onto the floating gate using this process. This can be done by applying a large negative voltage to the tunneling 


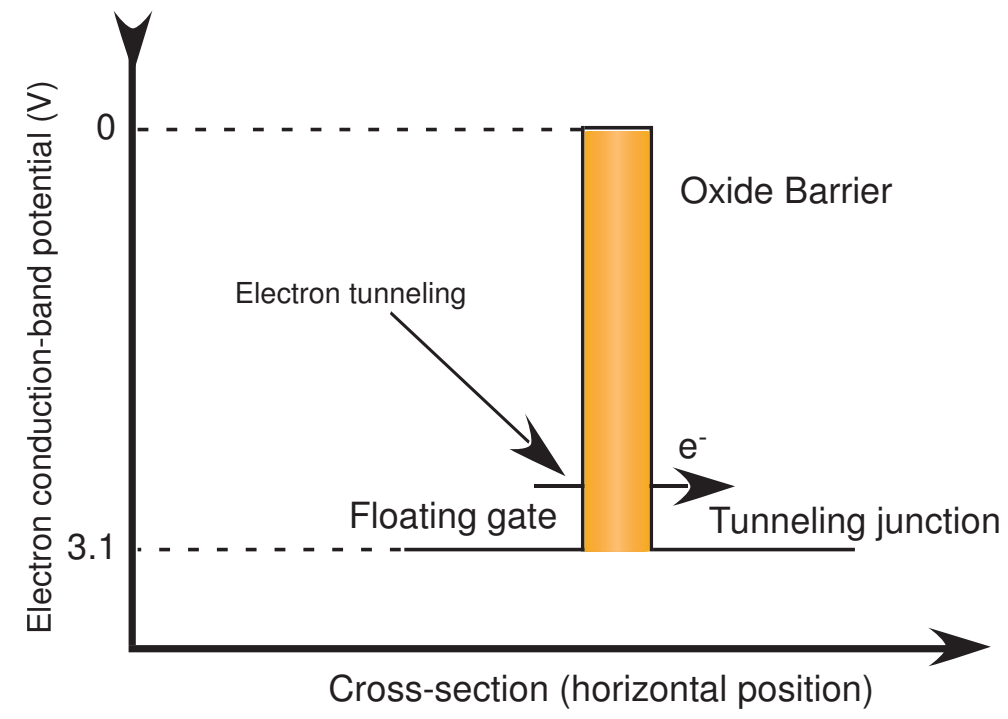

Figure 4.5: Electron tunneling

implant or by pulling the floating-gate to a high voltage. The first method is unsuitable for this as most processes are single-tub n-well and the second method causes large change in gate current. Tunneling can be used either to erase the memory on the floating gate or to program it to hold a particular charge on it. But, to selectively choose only one floatinggate among a matrix of floating-gates, only electron injection can be used. The electron injection process has two control voltages to enable injection and these two voltages can be used to select the row and column of the floating-gate. But, when using the tunneling process, there is no such control and it is generally used to remove the charge from the floating-gate. Therefore, electron tunneling is only used to completely 'erase' the memory (effectively the stored charge on the floating-gate) by removing the electrons from the gate. Electron injection is then used to inject electrons onto the floating-gate and precisely control the drain current. Large arrays of such floating-gates can be used to program the analog front-end or any such systems which require setting of lot of biases and changing them dynamically. 


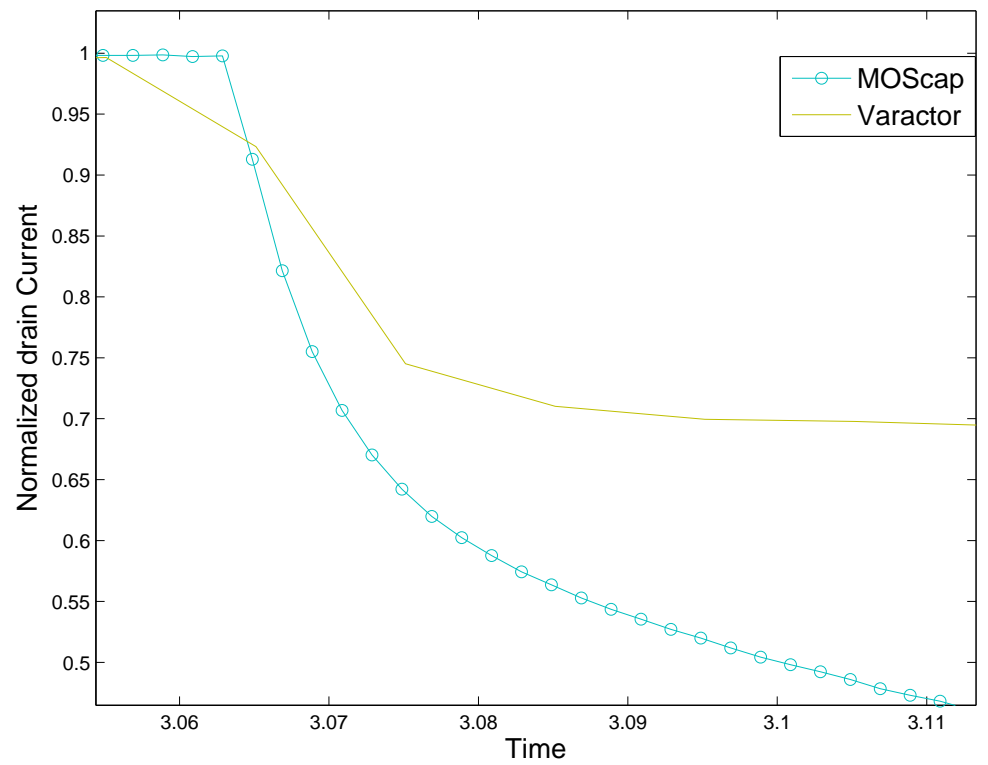

Figure 4.6: Comparison of tunneling times for MOScap and Varactor tunneling junctions

\subsubsection{Tunneling Junction}

A capacitor used to couple the tunneling voltage terminal to the floating gate is called a tunneling junction. This tunneling jucntion is normally built using a varactor in analog systems. But the different ways of building a tunneling junction include polysilicon-polysilicon capacitor, metal-inductor-metal capacitor and MOS capacitor. A MOS capacitor is comparatively better because of the substantially better oxide quality, resulting in improved reliablility. However, using a MOS capacitor as the tunneling capacitor results in faster tunneling. As illustrated in Figure 4.6 the drain current drops at a faster rate using a MOS capacitor than when using varactor as the tunneling junction. Therefore, a floating-gate matrix used to bias the 16 arrays in the analog front-end the tunneling junction can be built using a MOS capacitor. Figures 4.7 and 4.8 show the layout of the two types of tunneling junctions. 


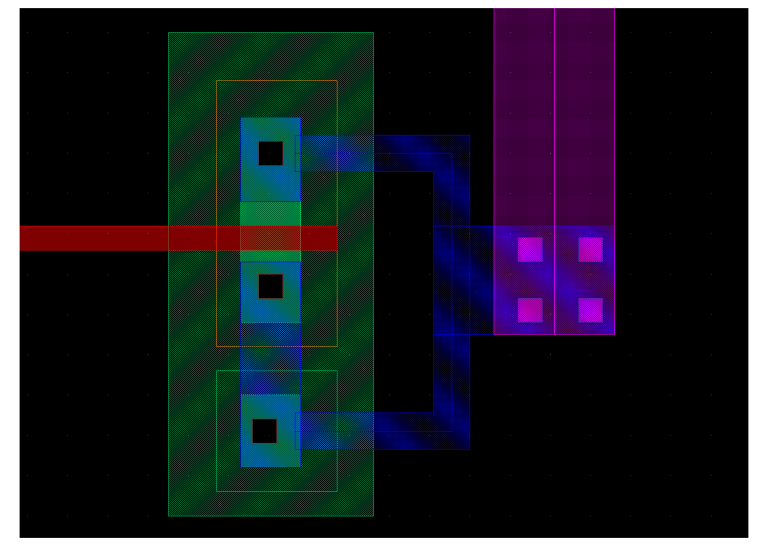

Figure 4.7: Layout of MOSCAP based tunneling junction

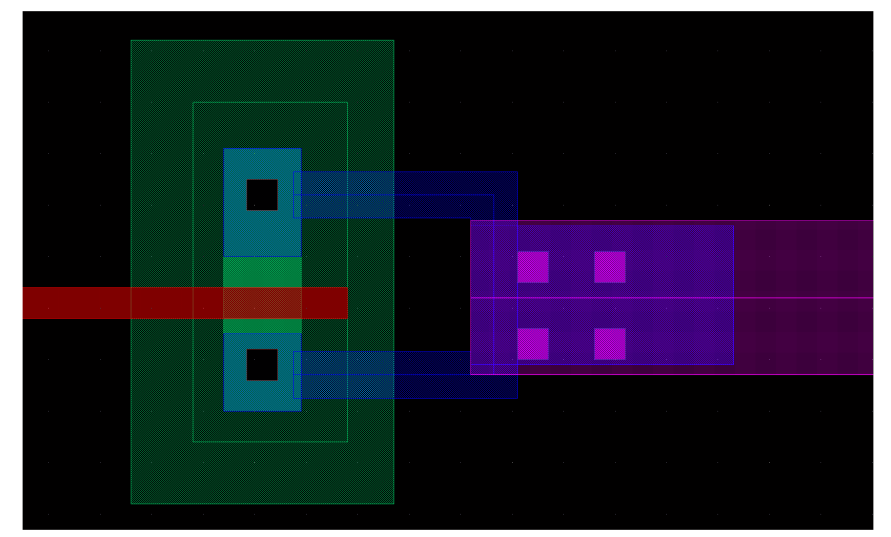

Figure 4.8: Layout of varactor based tunneling junction 


\section{Chapter 5}

\section{Analog Auditory Front-End}

This chapter explains the implementation of the analog front-end system by combining all the individual blocks designed and fabricated. Initially, as shown in Figure 1.2, the bandpass filter spectrally decomposes the signals and these signals are fed into sub-band processing blocks in different frequency taps. The application of these blocks entirely depends on the processing algorithm implemented by the end user. The front-end designed here has a total of 16 channels for real-time parallel processing of signals in different frequency ranges and is fabricted in a $0.18 \mu \mathrm{m}$ CMOS process.

\subsection{Working of Analog Auditory Front-End}

The analog front-end has 16 channels each of a band pass filter, magnitude detector, differentiator and voltage-mode subtractor. The design of the front-end is divided into two parts: the first part consists of the filter-bank and magnitude detector [17] and the second part consists of the rest of the circuits (Fig. 5.5). The filter-bank is used to spectrally decompose the audio signals received and the magnitude detector is used to extract the envelope of the signal. (Fig. 5.1 and 5.2). The spectrally decomposed signals from the band pass filter are sent to either the magnitude detector or the differentiator based on the audio processing algorithm. There is also an extra differentiator block in the system for obtaining the second derivative of the signals. This could be useful in applications where information about acceleration is required. And the voltage-mode subtractor circuit is 

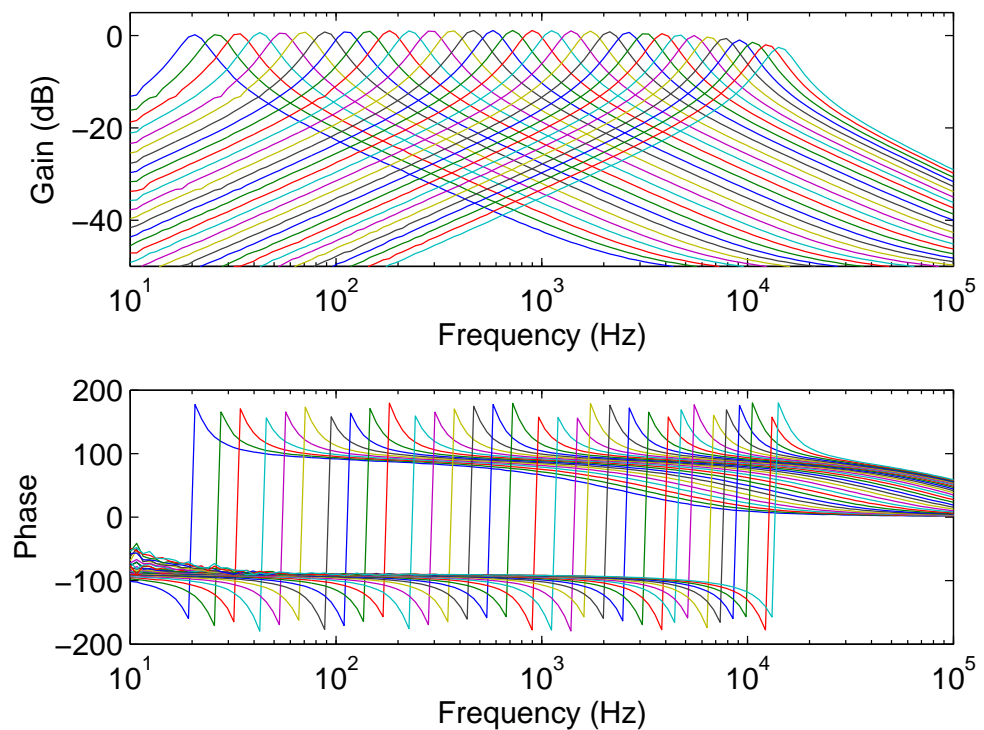

Figure 5.1: $C_{4}$ frequency response. The signals are spectrally decomposed using this circuit (simulated).

used to subtract either the magnitude-detector output or the differentiator output from the filter-bank output, thereby collecting information about the variations in the signals (Fig. 5.3 and 5.4) illustrate this. All the blocks are interconnected with multiplexing circuits to enable post-fabrication reconfigurability. This front-end comprising 16 channels (Fig. 1.2) is programed using floating-gate transistors described in Chapter 4. The layout of the complete system is shown in Figure 5.6. 


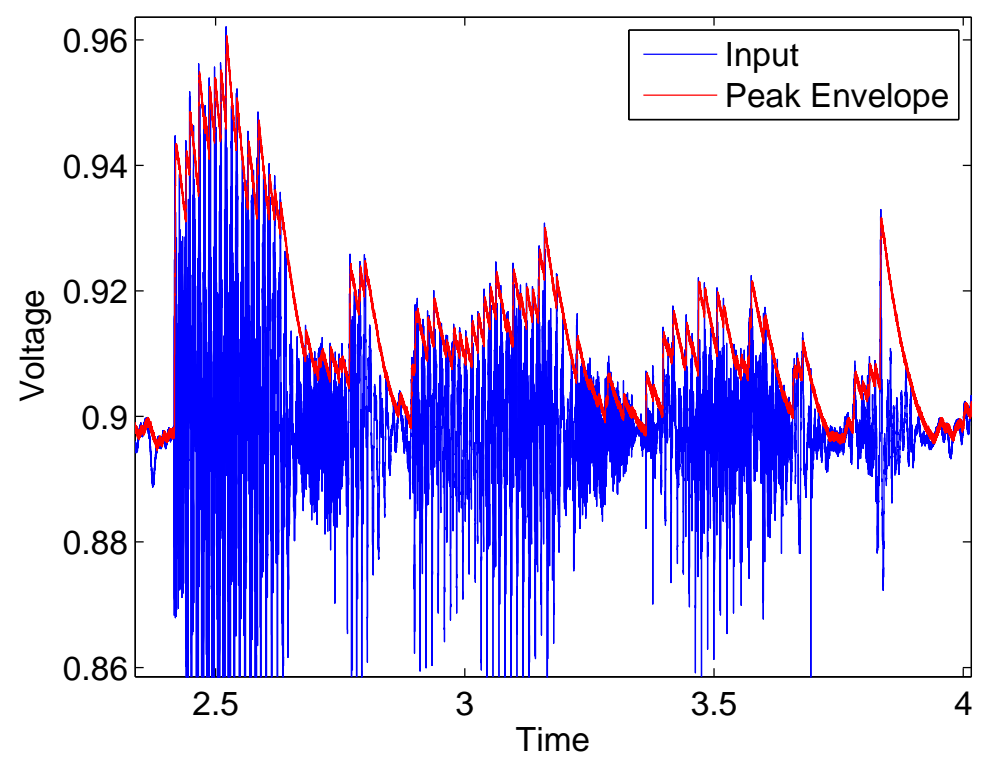

Figure 5.2: Response of the magnitude detector circuit (simulated).

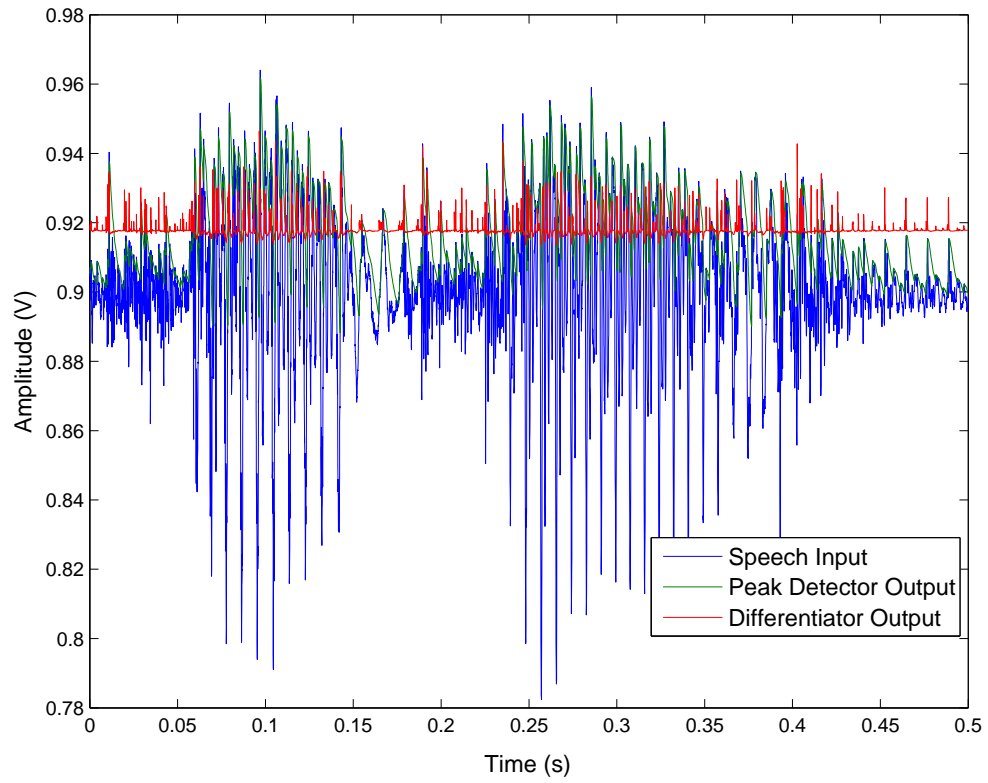

Figure 5.3: Respsonse of differentiator circuit when input is a peak detected signal (simulated). 


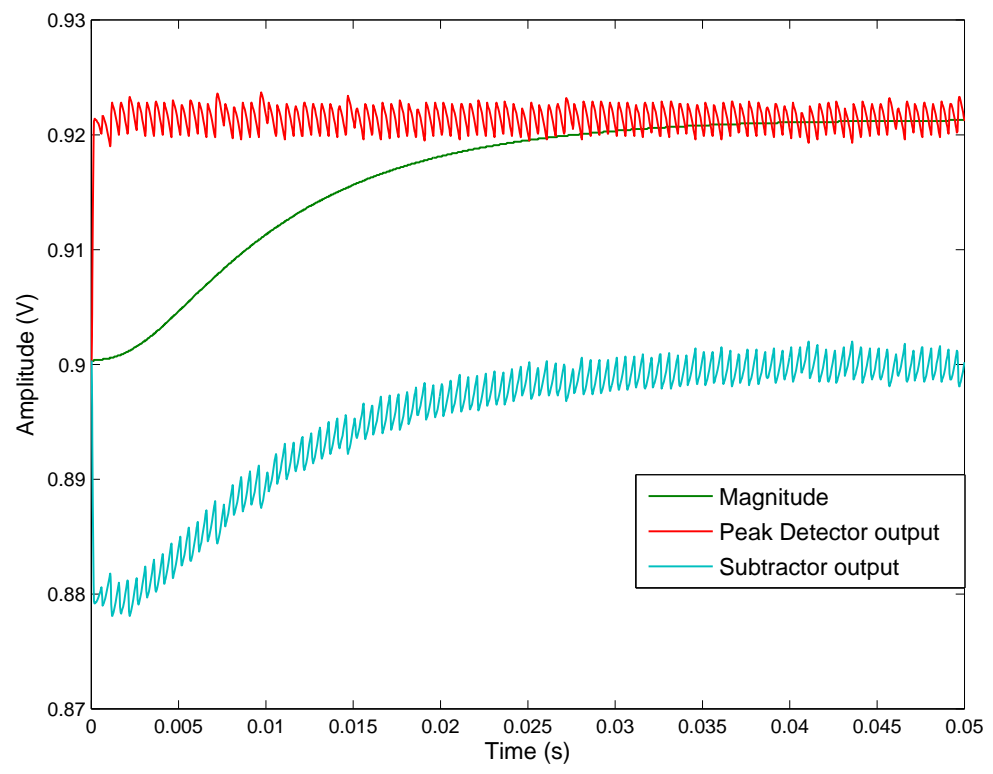

Figure 5.4: Transient response of subtractor circuit (simulated).

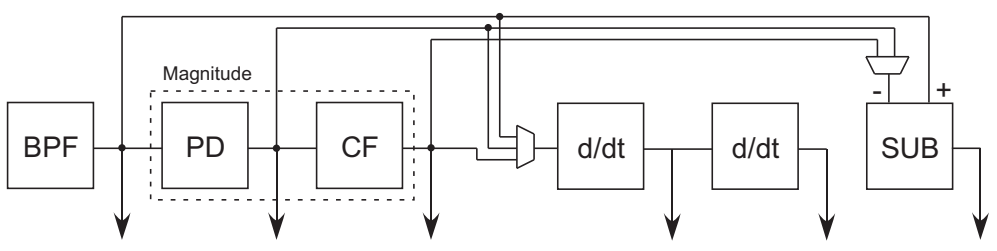

Figure 5.5: Single column of the analog front-end 


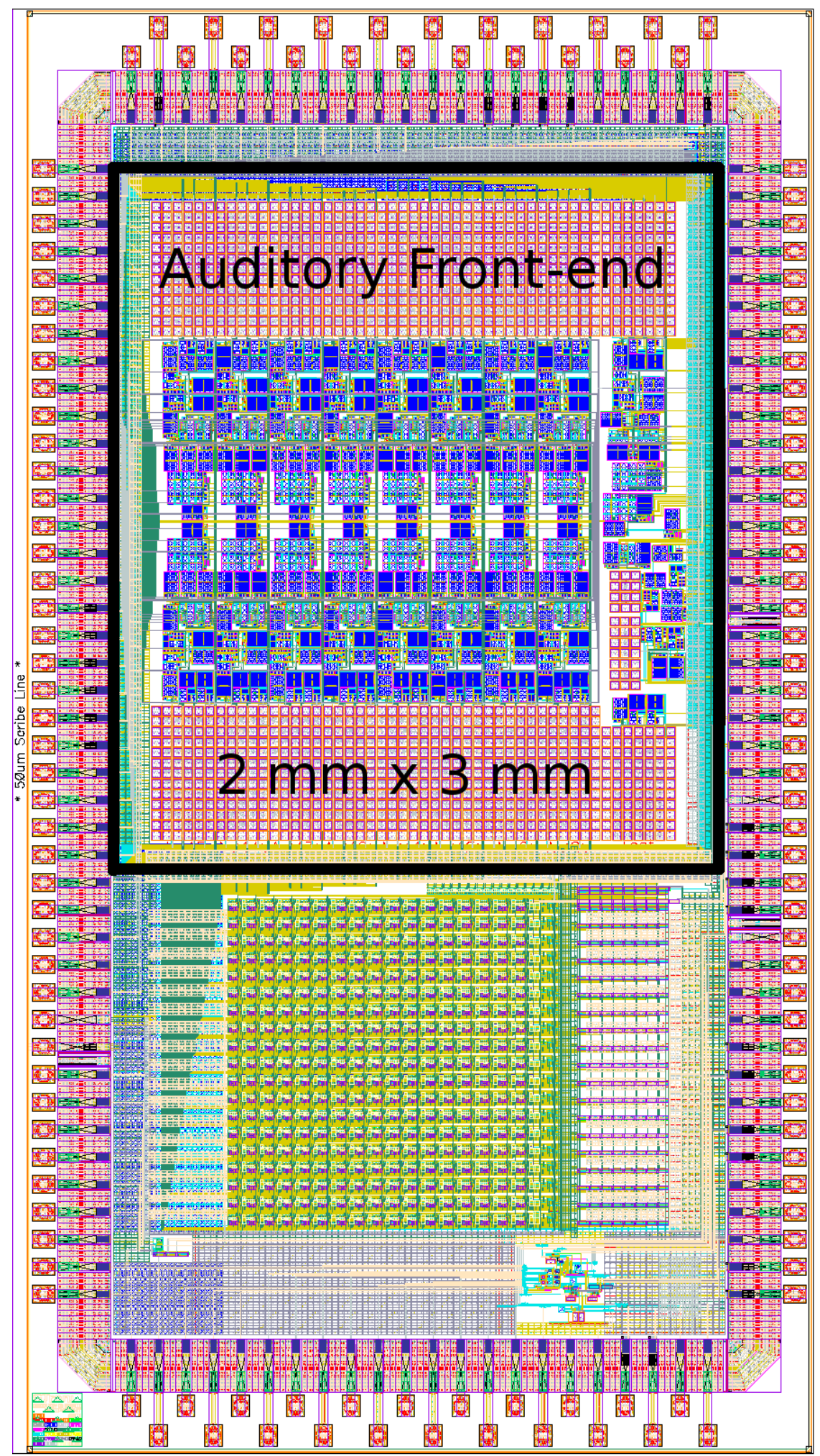

Figure 5.6: Layout of the $0.18 \mu m$ auditory front-end 


\section{Chapter 6}

\section{Applications and Conclusion}

\subsection{Low-Power Hardware-Based Event Detection}

The analog auditory front-end system desinged here finds many applications in speech recognition [18], sound localizing [19], noise supression [20] or hearing prosthesis [21]. One of the many application areas is wireless sensor networks. This is a very suitable field of application as power is at premium in such networks and also the contemporary sensor nodes essentially have low computational abilities [22, 23, 24, 25]. The analog systems here offer ultra-low-power alternatives when compared to digital counterparts for initial signal processing $[26,27]$. In addition to the low-power advantage, these systems also are able to perform some signal analysis and free-up the power hungry digital systems for other higherlevel tasks.

In sensor networks, usually the maximum power consumption is by the radio or the communication system. One good way to reduce this power is communicating only when required and also transmitting only the relevant information rather than everything the sensor detects. To acheive this, the radio is set to a sleep state until an event of interest is detected by the mote sensors [28]. To communicate only the relevant informaiton, some signal processing would be required on the mote itself and this is done by using the low-power analog circuits. This chapter explains the details of this application and its advantages. 


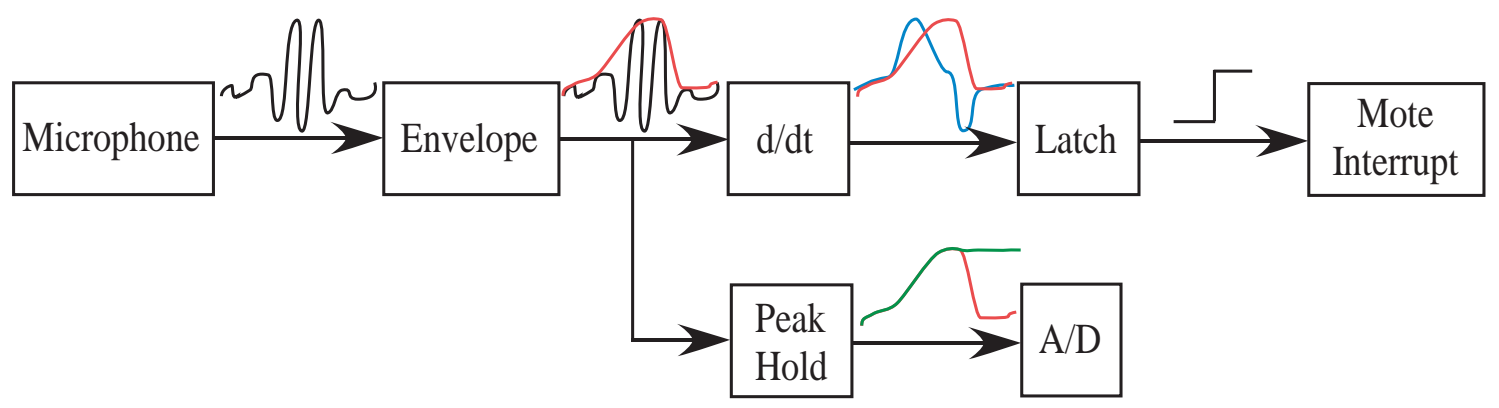

Figure 6.1: Idea for hardware detection of sound events.

\subsection{Design of the Analog System}

The main goal of this design was to use analog systems in the sensor motes to reduce the power consumption. The system here is designed to (1) detect an event based on a threshold value and (2) compare the signal input with a particular signature for classification of sounds [26]. In threshold-based detection, a wake-up signal is generated when the sensor input is higher than a particular threshold. An event is considered to have occured when this happens and the base-station is notified. In the sound classification application, the input is spectrally decomposed and a comparator reference is used for each sub-band to detect and classify events in different frequencies. For example, a foot step can be distinguished from a human voice. Figure 6.1 shows the basic idea for the circuit. The initial idea was to find the derivative of the magnitude to detect large changes, and then trigger a wake-up signal for the mote. But, the magnitude circuit used in the analog front-end described in [17] was not back from foundry, leading to the construction of peak detector using discrete elements. Also, as the output from the peak detector was not smooth and had a sawtooth shape (Fig. 6.1 ), it would result in false event triggering when fed to the differentiator circuit. Therefore, only the comparator was used as the main functional element for the first phase development of this application.

\subsubsection{Single-Dimensional Sound Localization}

To demonstrate this application, two circuits were built using discrete elements. Each of this was connected to IRIS motes via MDA300 sensor board. An event is considered to 


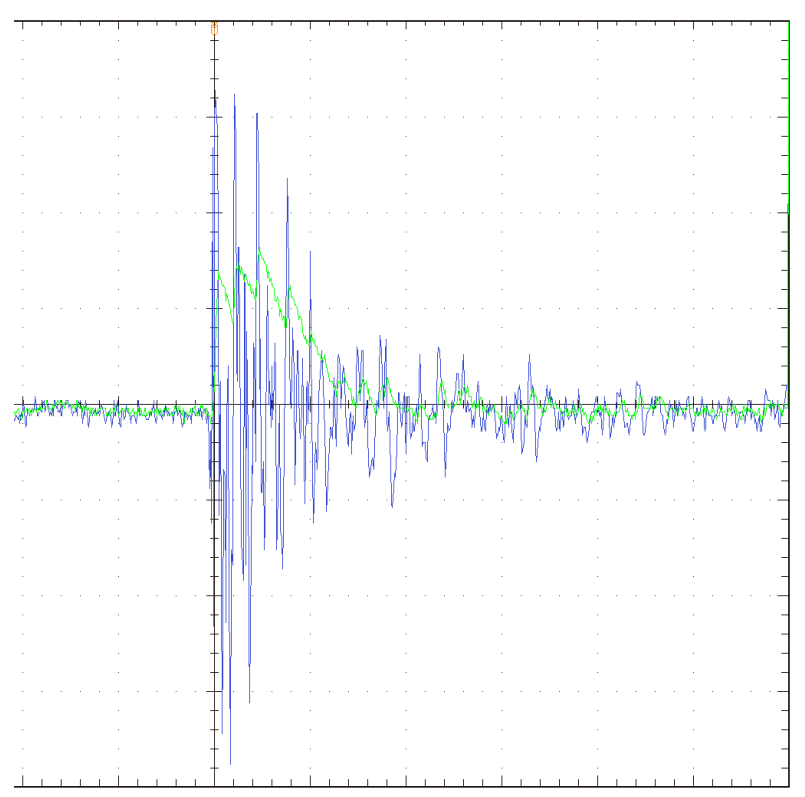

Figure 6.2: Response of the prototype peak detector circuit which was bread-boarded with discrete components.

have occured when the sensor input is above a threhold and the comparator output is used to trigger an interrupt to wake up the mote and transmit. A base station is connected to PC running MATLAB. This setup is used for one-dimensional sound localization. To synchronize the timers on the motes, the base station sends a packet every 10 seconds. When an event is detected, the motes are awakened by the trigger from comparator and transmit a packet containing the time of event to the base station. Based on this event-time from the two nodes, the direction of sound is determined in a single-dimension. This could be extended to multi-dimensional localization with more complex algorithms but as the focus here is to highlight the power savings in analog systems, it has only been done for a single-dimension. The total power consumption was about $3 m W$, for a system built with discrete elements.

\subsubsection{Sound/Event Classification}

For event-classification, a system with 8 channels of band pass filters, envelope detectors and comparators (Fig. 6.4) has been designed and fabricated in $0.5 \mu m$ process (Fig. 6.5). The signals are spectrally decomposed using the filterbanks and these signals are passed 


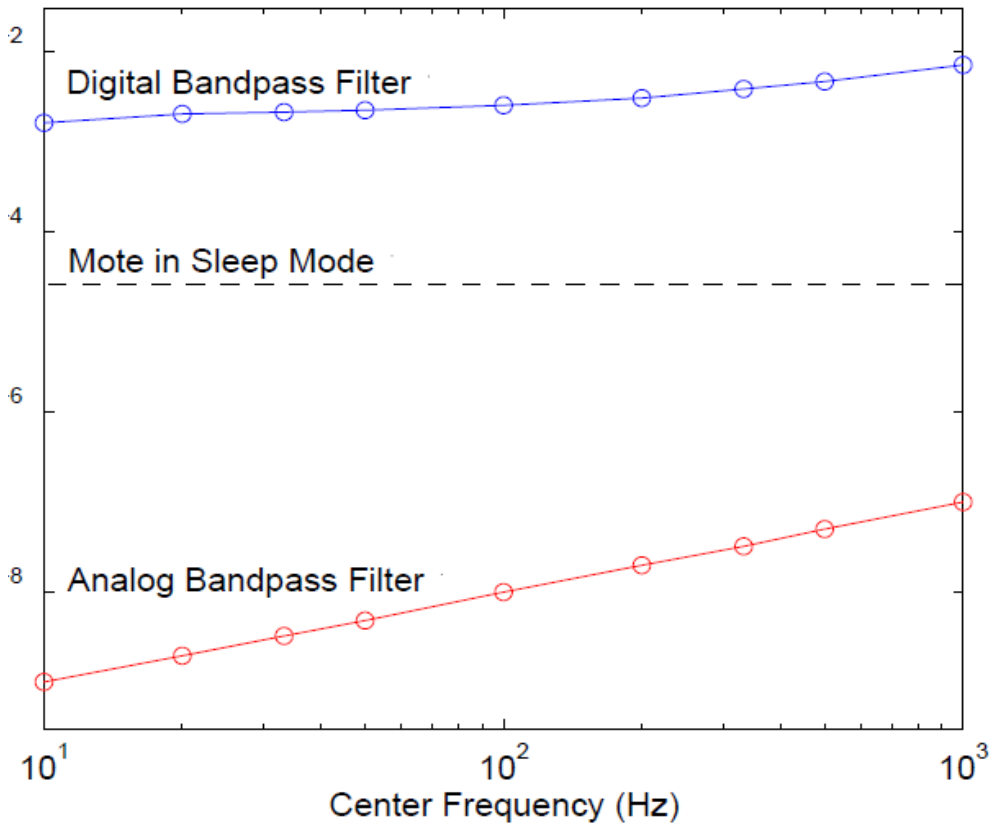

Figure 6.3: Comparison of average power consumed by analog bandpass filter and a digital bandpass filter $[26]$

through the envelope detectors to obtain the envelope of the signal. Each channel has a comparator and by using a suitable reference for this, the signals can be classified into different events. There is a latch for each channel to hold the sampled signal until the mote wakes up for transmission. Using this setup the motes can be used to listen for signals of only a particular frequency or to compare signals in two different frequencies. A few applications using such analog systems are reported in [26]. A similar digital system for such applications consumes $\approx 6 m W[26]$. Whereas, the analog system designed here consumes $\mu W s$ of power, which is thousand times less than its digital counterpart. Even a mote in sleep mode consumes more power than the analog system (Fig. 6.3) as shown in [26].

\subsection{Conclusion}

The design and working of analog circuits, particularly the differentiator and subtractor circuits for sub-band speech processing have been described in this work. These circuits are low-power and tunable for various operating frequencies within the audio range. An array of 16 channels has also been fabricated to allow real-time parallel signal processing at various 


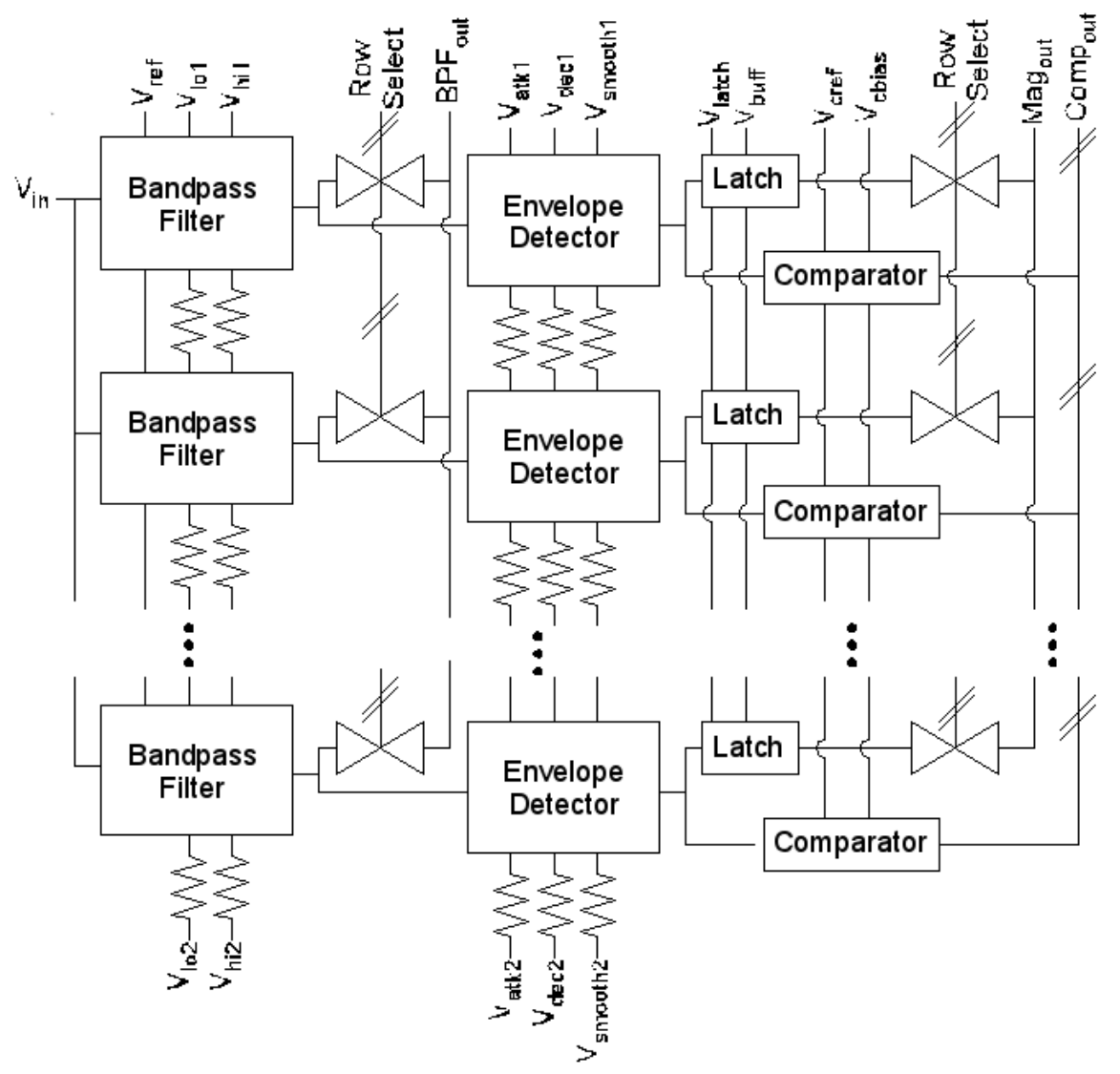

Figure 6.4: Block diagram for the fabricated event detection system.

frequencies. These front-ends when integrated with a digital signal processor could lead to intersting applications like low-power sensors, audio noise supression, voice recognition and event-classification based on the sound signals. Also, as all the blocks in the front-end are connected using switching circuits, they could be reconfigured in different ways to suit a wide range of algorithms for signal processing. In future, these individual blocks could also be integrated onto an Analog Signal Processor (ASP) [29] or a Field Programable Analog Array (FPAA) [30] as basic building elements or into configurable analog blocks (CABs). Even complex functions can be synthesized by using such CABs. The next step would be to design more such basic biulding blocks for sub-band processing and integrating them with ASPs or CABs. 


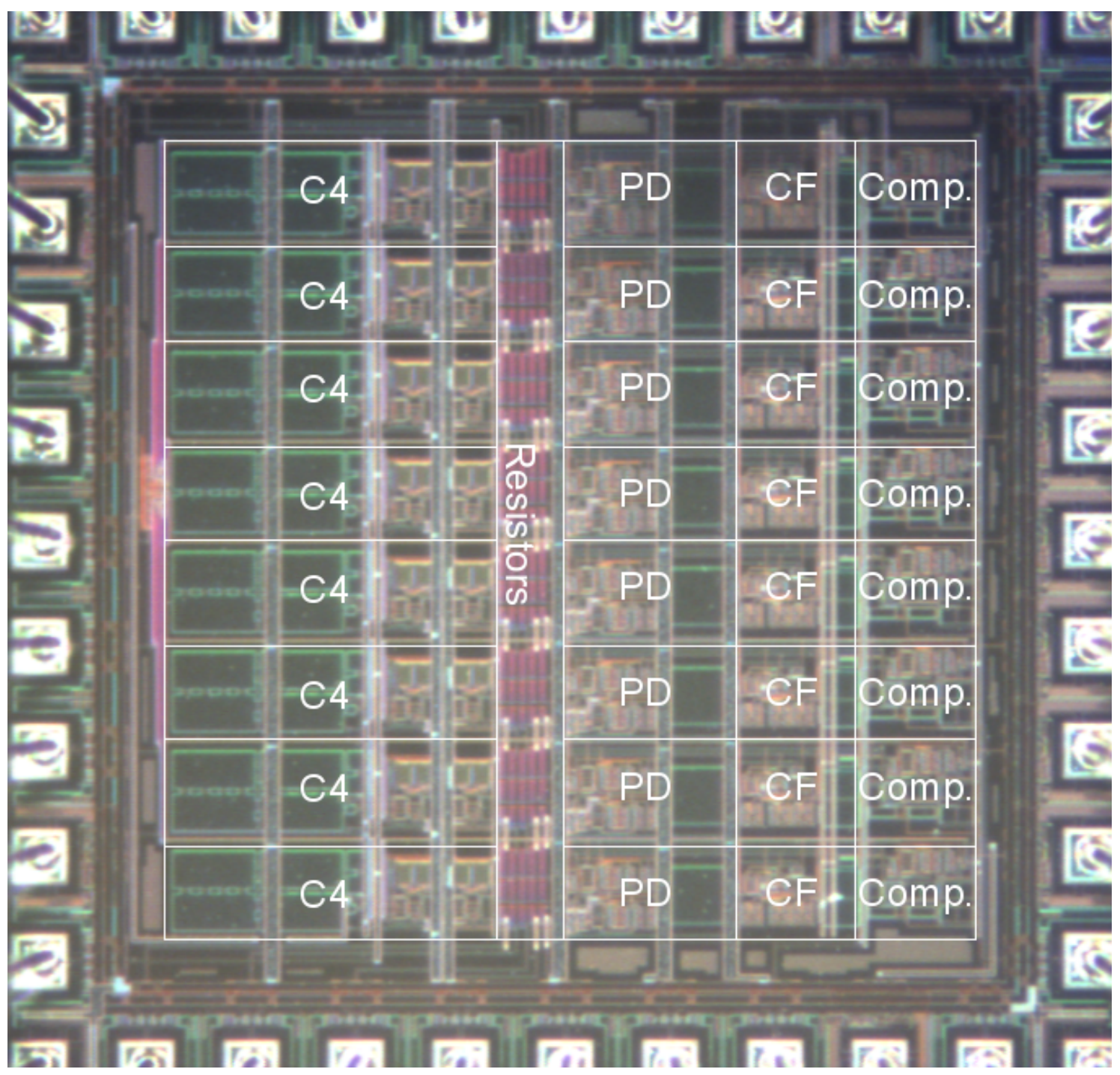

Figure 6.5: Die shot of the event detection chip. The area inside the pad frame measures $1.5 \mathrm{~mm} \times 1.5 \mathrm{~mm}$ 


\section{Appendix A}

\section{Cadence setup}

Majority of the work in this thesis was done on the software suite from Cadence Inc. It was used to run simulaitons, do the layout of circuits in various CMOS processes and also build PCBs to test the fabricated ICs. This software suite was setup on systems with Ubuntu (an open souorce version of Linux operating system), which is not officially supported by Cadence. To do this, various customizations had to be done to make it work on this particular version of Linux operating system. The final goal was to setup this software such that it could be accessed from all the departmental laboratories. Initially a CentOS based system was used as the license server but eventually, all the applications in the suite and the license server were migrated to Ubuntu based operating system at West Virginia University, known as LOUD (LCSEE Optimized Ubuntu Distribution). Presently this software suite can be accessed from any of the departmental laboratories. 


\section{Appendix B}

\section{More Derivative Circuit Models}

\section{B.1 Hysteretic Differentiators}

A few more circuit models considered for derivative circuit are the Hysteretic Differentiators. While the models described in Chapter 2 were all based on linear systems, this set of circuits are based on non-linear systems for obtaining the differentiator output. This works on the basis of the basic property of differentiation, in which a steady value or small changes in the input are suppressed while large changes are amplified.

The hysteretic circuits use only one amplifier and a non-linear element, like a diodeconnected MOSFET in the feedback path, to perform differentiation (Fig. B.1) [3, 5]. With this setup, the circuit will show expansive non-linearity. That is, the small signals have larger resistance and larger signals have lower resistance due to the diode connected MOSFET in the feedback path. If the feedback path has two diode-connected MOSFETs in its feedback

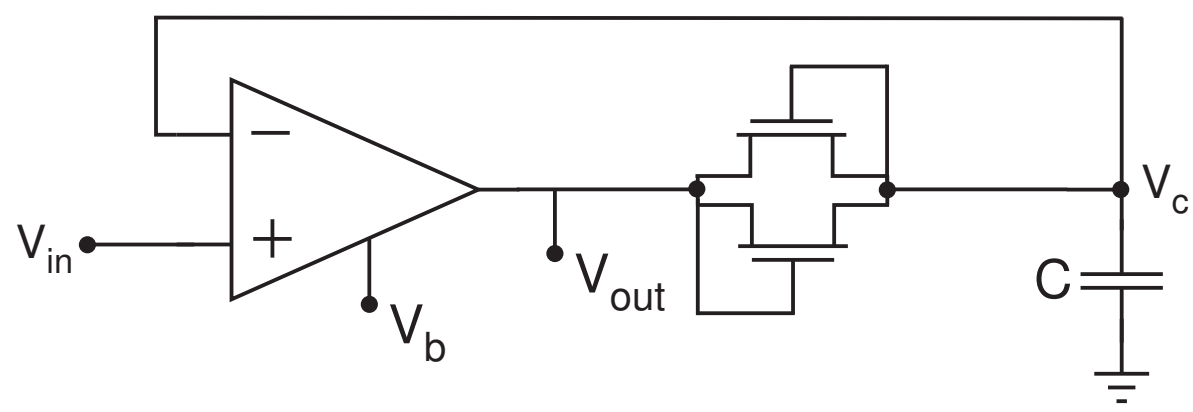

Figure B.1: Hysteretic differentiator circuit 
path to support the positive and negative swings of input signals, this can be used as a differentiator. Theoretically, this setup works as a differentiator but it is not suitable for the particular application for this project. It differentiates large signals but not the small signals. In effect, it only shows changes in sign of the input signal. Any small changes would be attenuated. Various other hysteretic differentiator circuits, as shown in Figure B.2, have been considered for this project. Each of these circuits uses the same basic principle of attenuating small changes and amplifying large changes. Therefore, none of the circuits meet the basic requirement of differentiating signals of various amplitudes over audio range from $20 \mathrm{~Hz}$ to $20 \mathrm{kHz}$. Also it does not satisfy the requirement of having a unity gain for the output irrespective of the input signal amplitude. Thus, even this circuit model would not be useful for building a differentiator circuit for this project.

\section{B.2 RL-Type Differentiators}

The differentiator circuit can also be modeled based on a Resistor-Inductor type highpass filter. To model this, the basic Tow-Thomas biquad form can be used. This RL-type circuit requires four Operational Transconductance Amplifiers (OTAs). The resistor can be modeled using a "resistor-connected" OTA and the inductor can be modeled by impedance inverter as shown in Figure B.3. There are two options for the input-stage of this circuit: follower-connected OTA or an open-loop OTA. The follower connected OTA gives low gain at the frequency of interest, but the THD is decent. The open-loop OTA (either inverting or non-inverting) gives a good gain at the frequency of interest but the THD values are very high. Also, as the input stage is in open-loop configuration, any shift in input DC voltage can cause stability problems. Due to the low-gain or high THD problems this circuit can not be used for the application in this project. 

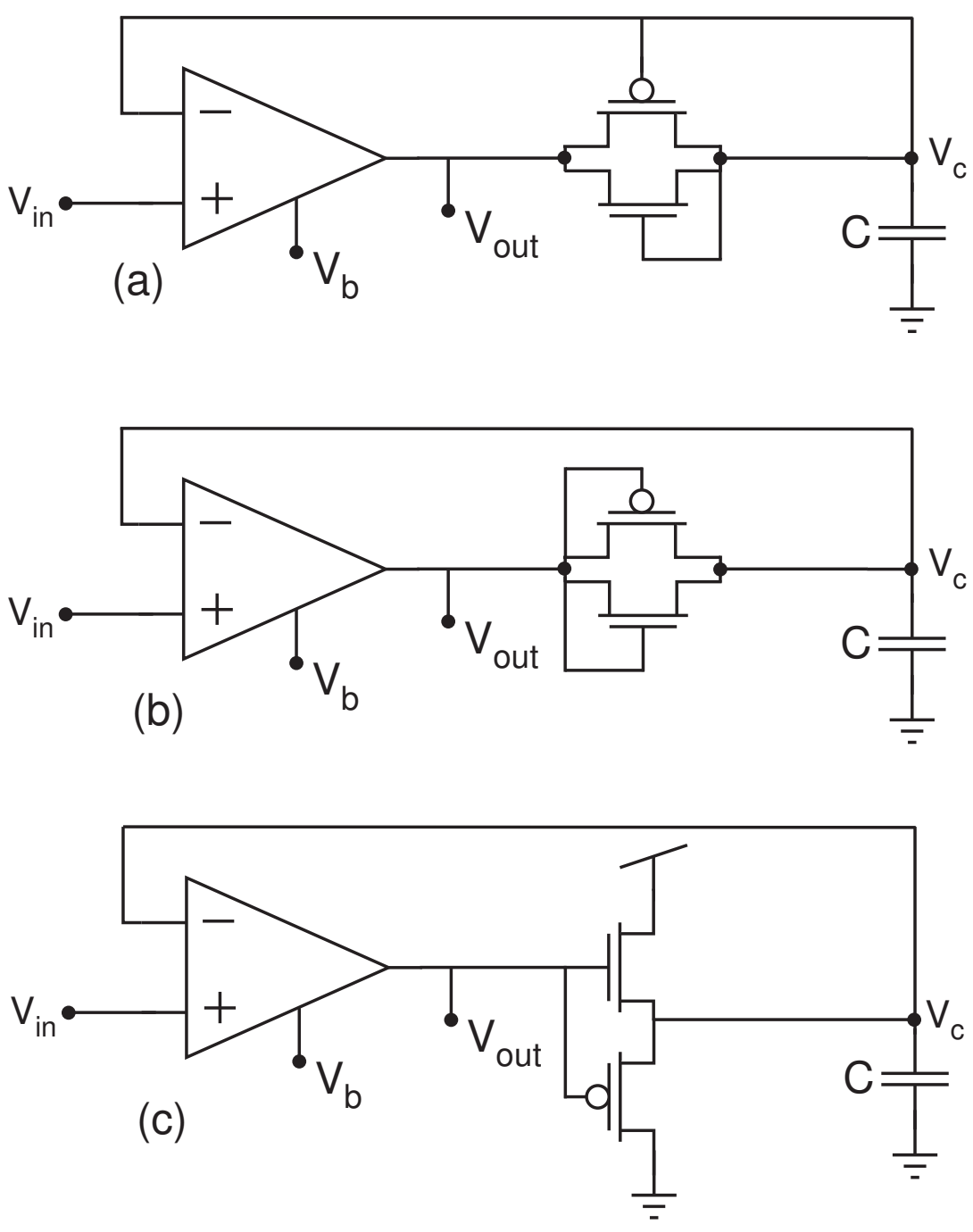

Figure B.2: Different versions of the hysteretic differentiator circuit. (a) The capacitor charges through the pFET and discharges through nFET. (b) It works the same as circuit in (a) but the has a higher peak-to-peak amplitude than (a) for the same variation in input. (c) Another variation for charging and discharging the capacitor but it does not satisfy the requirements for this project 


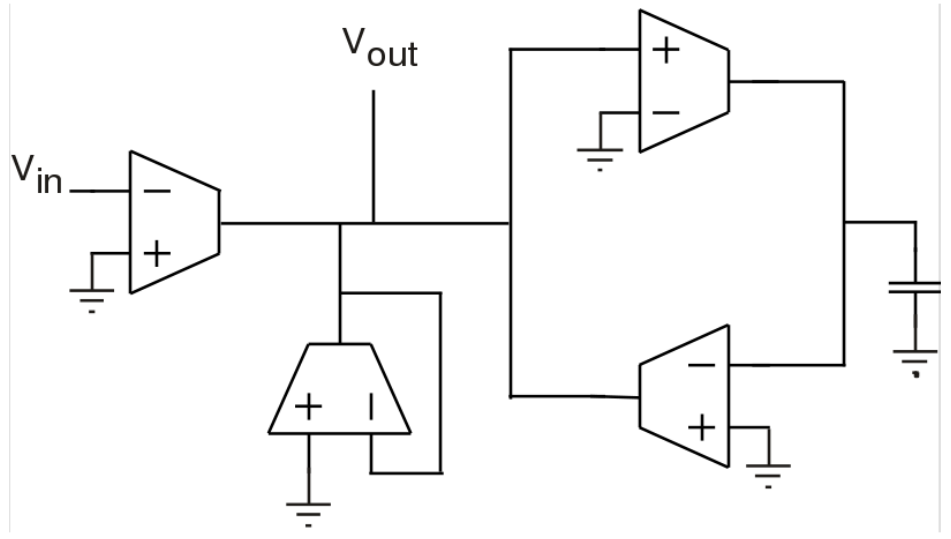

Figure B.3: The RL-type differentiator 


\section{References}

[1] Behzad Razavi, Design of analog CMOS integrated circuits, McGraw Hill, 2001.

[2] Ivan A. Greenwood Jr., J. Vance Holdam Jr., and Duncan Machrae Jr., Electronic Instruments, volume 21 of MIT Radiation Laboratory Series, McGraw-Hill, 1948.

[3] C. Mead, Analog VLSI and Neural Systems, Addison-Wesley, 1989.

[4] R. Fried and C.C. Enz, "Simple and accurate voltage adder/subtractor," in Electronics Letters, May 1997, vol. 33, pp. 944-945.

[5] Jorg Kramer, Giacomo Indiveri, Tobias Delbruck, Shih-Chii Liu, and Rodney Douglas, Analog VLSI: Circuits and Principles, The MIT Press, 2002.

[6] Eiji Takeda, Cary Yang, and Akemi Miura-Hamada, Hot-Carrier effects in MOS devices, vol. optional of optional, Academic Press, Elsevier, optional, optional edition, November 1995, optional.

[7] M. Lenzlinger and E. H. Snow, "Fowler-Nordheim Tunneling into Thermally Grown $\mathrm{SiO}_{2}, "$ Journal of Applied Physics, vol. 40, pp. 278-283, Jan. 1969.

[8] C. Enz, F. Krummanacher, and E. Vittoz, "An analytical mos transistor model valid in all regions of operation and dedicated to low-voltage and low-current applications," Journal on Analog Integrated Circuits and Signal Processsing, , no. 1, pp. 83-114, July 1995.

[9] David W. Graham and Paul Hasler, "Capacitively-coupled current conveyer secondorder section for continuous-time bandpass filtering and cochlea modeling," in Proceedings of the IEEE ISCAS, Atlanta, GA, 2002.

[10] A. Bouzerdoum, A. Yakovleff, D. Abbott, O. Kim, K. Eshraghian, A. Moini, and R.E. Bogner, "An analog implementation of early visual processing in insects," in Proceedings of International Symposium on VLSI Technology, Systems and Applications, 1993, pp. 283-287.

[11] T. Delbruck and C. Mead, "Analog vlsi adaptive logarithmic wide-dynamic-range photoreceptor," in Proceedings of the IEEE ISCAS, 1994, pp. 339-342.

[12] E.A. Vittoz, Analog VLSI computation of neural networks: Handbook of Neural Computation, Institute of Pyhsics Publishing and Oxford Univresity Press, USA. 
[13] A.S. Sedra and G.W. Roberts, "Current conveyor theory and practice," Advances in Analog Integrated Circuit Design, pp. 93-126, 1990.

[14] M.G. Johnson, "An input-free vt extractor circuit using a two-transistor differential amplifier," IEEE Journal of Solid-State Circuits, vol. 28, pp. 704-705, 1993.

[15] E.A. Vittoz, Micropower Techniques: Principles of Neural Science, Prentice-Hall, 1994.

[16] Douglas A. Kerns, John E. Tanner, Massimo A. Sivilotti, and Jin Luo, "Cmos uvwritable non-volatile analog storage," in Proceedings of the 1991 University of California/Santa Cruz conference on Advanced research in VLSI, Cambridge, MA, USA, 1991, pp. 245-261, MIT Press.

[17] Brandon Rumberg, "Speech processing front-end in low-power hardware," M.S. thesis, West Virginia University, Morgantown, West Virginia, 2009.

[18] T. Massengill, D. Wilson, P. Hasler, and D. W. Graham, "Empirical comparison of ana$\log$ and digital auditory preprocessing for automatic speech recognition," in Proceedings of the IEEE International Symposium on Circuits and Systems, Scottsdale, AZ, May 2002, vol. 5, pp. 77-80.

[19] A. van Schaik and S. Shamma, "A neuromorphic sound localizer for a smart MEMS system," in Proceedings of the International Symposium on Circuits and Systems, May 2003, vol. 4, pp. IV-864-IV-867.

[20] R. Ellis, H. Yoo, D. W. Graham, P. Hasler, and D. V. Anderson, "A continuous-time speech enhancement front-end for microphone inputs," in Proceedings of the IEEE International Symposium on Circuits and Systems, Scottsdale, AZ, May 2002, vol. 2, pp. II-728-II-731.

[21] Rahul Sarpeshkar, Christopher Salthouse, Ji jon Sit, Michael W. Baker, Serhii M. Zhak, Timothy K. -t. Lu, Lorenzo Turicchia, and Stephanie Balster, "An ultra-low-power programmable analog bionic ear processor," in IEEE Transactions on Biomedical Engineering, 2005, pp. 711-727.

[22] Anish Arora, Rajiv Ramnath, Emre Ertin, Prasun Sinha, Sandip Bapat, Vinayak Naik, and Vinod Kulathumani, "Exscal: Elements of an extreme scale wireless sensor network," in RTCSA '05: Proceedings of the 11th IEEE International Conference on Embedded and Real-Time Computing Systems and Applications, Washington, DC, USA, 2005, pp. 102-108, IEEE Computer Society.

[23] Marco F. Duarte and Yu Hen Hu, "Vehicle classification in distributed sensor networks," Journal of Parallel and Distributed Computing, vol. 64, no. optional, pp. 826-838, July 2004, optional.

[24] V. Raghunathan, C. Schurgers, Sung Park, and M.B. Srivastava, "Energy-aware wireless microsensor networks," Signal Processing Magazine, IEEE, vol. 19, no. 2, pp. 40-50, mar 2002. 
[25] Ning Xu, Sumit Rangwala, Krishna Kant Chintalapudi, Deepak Ganesan, Alan Broad, Ramesh Govindan, and Deborah Estrin, "A wireless sensor network for structural monitoring," in SenSys '04: Proceedings of the 2nd international conference on Embedded networked sensor systems, New York, NY, USA, 2004, pp. 13-24, ACM.

[26] Brandon Rumberg, David Graham, and Vinod Kulathumani, "Hibernets: Energyefficient sensor networks using analog signal processing," in Proceedings of International Conference on Information Processing in Sensor Networks, Stockholm, Sweden, 2010 (accepted).

[27] Rahul Sarpeshkar, "Analog versus digital: extrapolating from electronics to neurobiology," Neural Comput., vol. 10, no. 7, pp. 1601-1638, 1998.

[28] S. Jevtic, M. Kotowsky, R.P. Dick, P.A. Dindap, and C. Dowding, "Lucid dreaming: reliable analog event detection for energy-constrained applications," Piscataway, NJ, USA, 2007/04/, pp. 350-359.

[29] P. Hasler, P. Smith, D. Graham, R. Ellis, and D. Anderson, "Analog floating-gate, onchip auditory sensing system interfaces," IEEE Sensors Journal, vol. 5, pp. 1027-1034, October 2005.

[30] T.S. Hall, C.M. Twigg, J.D. Gray, P. Hasler, and D.V. Anderson, "Large-scale fieldprogrammable analog arrays for analog signal processing," Circuits and Systems I: Regular Papers, IEEE Transactions on, vol. 52, no. 11, pp. 2298-2307, nov. 2005. 


\title{
Low-Power Analog Circuits for Sub-Band Speech Processing
}

\author{
by \\ Anvesh Kumar Singireddy \\ Thesis submitted to the \\ College of Engineering and Mineral Resources \\ at West Virginia University \\ in partial fulfillment of the requirements for the degree of \\ Master of Science \\ in \\ Electrical Engineering
}

Lane Department of Computer Science and Electrical Engineering

APPROVAL OF THE EXAMINING COMMITTEE

Dimitris Korakakis, Ph.D.

Jeremy Dawson, Ph.D.

David W. Graham, Ph.D., Chair

Date

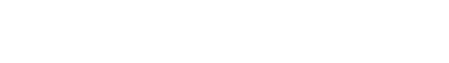

Hagen 